\title{
Sharp Error Term in Local Limit Theorems and Mixing for Lorentz Gases with Infinite Horizon
}

\author{
Françoise Pène $^{1}$, Dalia Terhesiu ${ }^{2}$ \\ ${ }^{1}$ Univ Brest, Université de Brest, LMBA, UMR CNRS 6205, 6 Avenue Le Gorgeu, 29238 Brest cedex, \\ France. E-mail: francoise.pene@univ-brest.fr \\ 2 Mathematisch Instituut, University of Leiden, Niels Bohrweg 1, 2333 Leiden, CA, The Netherlands. \\ E-mail: d.e.terhesiu@math.leidenuniv.nl
}

Received: 4 February 2020 / Accepted: 15 January 2021

Published online: 17 February 2021 - (C) The Author(s) 2021

\begin{abstract}
We obtain sharp error rates in the local limit theorem for the Sinai billiard map (one and two dimensional) with infinite horizon. This result allows us to further obtain higher order terms and thus, sharp mixing rates in the speed of mixing of dynamically Hölder observables for the planar and tubular infinite horizon Lorentz gases in the map (discrete time) case. We also obtain an asymptotic estimate for the tail probability of the first return time to the initial cell. In the process, we study families of transfer operators for infinite horizon Sinai billiards perturbed with the free flight function and obtain higher order expansions for the associated families of eigenvalues and eigenprojectors.
\end{abstract}

\section{Introduction}

Lorentz gas. The Lorentz gas has been introduced in [18] to model the displacement of electrons in metals. This model describes the evolution of a point particle moving freely with unit velocity and elastic reflections off pairwise disjoint strictly convex obstacles (with smooth boundary) located $\mathbb{Z}^{d}$-periodically (with $d \in\{1,2\}$ ) in the plane if $d=2$ or on the tube $\mathbb{T} \times \mathbb{R}=\mathbb{R}^{2} /(\mathbb{Z} \times\{0\})$. These obstacles are written $\mathcal{O}_{j}+\ell$ with $j=1, \ldots, \mathcal{J}$ and $\ell \in \mathbb{Z}^{d}$ (where $\mathcal{J}$ a non empty finite set). In this model, the phase space consists of positions-unit velocity vectors, which we call configurations (Figs. 1, 2).

In this paper, we are interested in discrete time Lorentz gases (the dynamical system corresponding to the collision times) with infinite horizon. The horizon is said to be infinite if there exists an infinite trajectory intersecting no obstacle and it is said to be finite otherwise. Understanding the stochastic behaviour of the Lorentz gas in the infinite horizon case is much more challenging than the finite horizon case and below we recall the main differences along with previous results. The exposition below focuses on the $\mathbb{Z}^{2}$-periodic case, but we mention that similar statements hold for $\mathbb{Z}$-periodic tubular model.

The space $M$ of configurations of this dynamical system is the set of postcollisional unit vectors based on $\bigcup_{j \in \mathcal{J}, \ell \in \mathbb{Z}^{2}} \partial \mathcal{O}_{j}+\ell$. For any $\ell \in \mathbb{Z}^{2}$, we define the $\ell$-th cell $M_{\ell}$ 


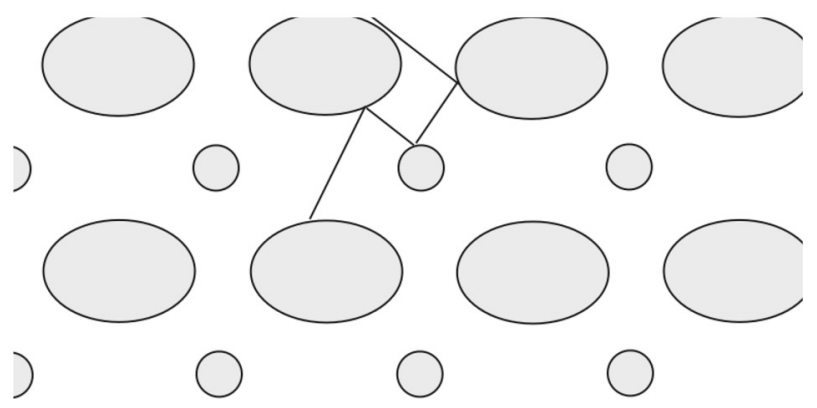

Fig. 1. A trajectory in a $\mathbb{Z}^{2}$-periodic planar domain $(d=2)$

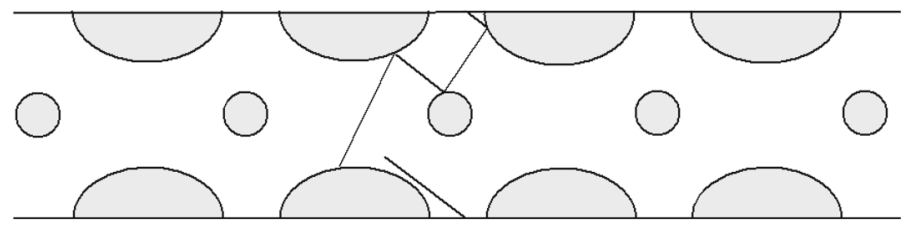

Fig. 2. A trajectory in a $\mathbb{Z}$-periodic tubular domain $(d=1)$

as the set of configurations with position in $\bigcup_{j \in \mathcal{J}} \mathcal{O}_{j}+\ell$. We write $\kappa_{n}$ for the label in $\mathbb{Z}^{2}$ of the cell in which the particle is at the $n$-th collision time:

$\kappa_{n}=N$ if the position of the particle is based in $\bigcup_{j \in \mathcal{J}} \mathcal{O}_{j}+N$ at the $n$th collision time

The finiteness of the horizon is equivalent to the uniform boundedness of $\kappa_{1}$. Whereas the model we consider is purely deterministic (position and velocity at collisions can be computed explicitly in terms of the initial one), $\left(\kappa_{n}\right)_{n \geq 0}$ behaves asymptotically as a random walk on $\mathbb{Z}^{2}$. In the finite horizon case, $\left(\kappa_{n}\right)_{n \geq 0}$ behaves asymptotically as a simple symmetric random walk, while in the infinite horizon we have a symmetric random walk with displacement of infinite variance. It is worth noticing that the dynamics of the discrete time Lorentz gas is given by the sequence of couples $\left(X_{n}, \kappa_{n}\right)_{n \geq 1}$ where $X_{n}$ with values in $M_{0}$ is the configuration modulo $\mathbb{Z}^{2}$ of the position at the $n$-th collision time. More precisely,

$X_{n}=(q, \vec{v}) \in M_{0}$ if the configuration at the $n$-th collision time is $\left(q+\kappa_{n}, \vec{v}\right)$.

In this representation $\left(\kappa_{n}\right)_{n}$ corresponds to the evolution of the Lorentz gas at a macroscopic scale while $\left(X_{n}\right)_{n}$ corresponds to its evolution at a microscopic scale. The dynamics of $\left(X_{n}\right)_{n}$ is refereed to as Sinai billiard and recall that the ergodicity of this dynamical system has been established in the seminal work by Sinai [28]. Several limit laws for Lorentz process are obtained for the invariant probability measure $\mathbb{P}=\bar{\mu}$ on $M_{0}$ absolutely continuous with respect to Lebesgue and for which $\left(X_{n}\right)_{n \geq 0}$ and $\left(\kappa_{n+1}-\kappa_{n}=\kappa\left(X_{n}\right)\right)_{n \geq 0}$ is a stationary process. Under this invariant probability measure, $\kappa_{1}$ is not square integrable when the horizon is infinite, whereas it is (as already mentioned) bounded when the horizon is finite.

Limit properties of discrete time finite horizon Lorentz gases have been obtained in several recent works, among which we mention [21-25,29]. Very recent notable progress 
for continuous time Lorentz gases with finite horizon has been made for mixing local limit theorem by Dolgopyat and Nandori $[9,10]$, for mixing rates by Dolgopyat, Nandori, Pène [11] and for suitable versions of CLT by Pène and Thomine [25]. Obtaining the analogue of any the said results in the infinite horizon case is very challenging because $\kappa_{1}$ has infinite variance. The main difficulty in carrying out similar arguments when $\kappa_{1}$ has infinite variance comes down to the weak regularity properties of a family $\left(P_{t}\right)_{t}$ of perturbed operators. Whereas in the finite horizon case eigenelements of these operators are $C^{\infty}$ in $t$, in the infinite horizon case the family of operator is just continuous in $t$ as an operator from the Young Banach space $\mathcal{B}$ to $L^{p}$ (see additional explanations in Sect. 3. In this paper we obtain refined expansions going beyond mere continuity estimates and use this to answer unsolved problems in the discrete time model with infinite horizon. In particular, we obtain: (i) higher order (optimal) local limit theorem and mixing; (ii) first order expansion of the tail probability of the first return time to the initial cell. In what follows we provide a simplified statement of our main results, recalling and comparing with previous results.

Previous results: CLT and local limit theorem $(L L T)$ for $\kappa_{n}$. When the horizon is finite, it has been proved in $[5,6,32]$ that $\left(\kappa_{n}\right)_{n}$ behaves asymptotically as a Gaussian random variable with the standard normalization in $\sqrt{n}$, that is

$\forall A_{i}<B_{i}, \quad \lim _{n \rightarrow+\infty} \mathbb{P}\left(\frac{\kappa_{n}}{\sqrt{n}} \in\left[A_{1}, B_{1}\right] \times\left[A_{2}, B_{2}\right]\right)=\mathbb{P}\left(W \in\left[A_{1}, B_{1}\right] \times\left[A_{2}, B_{2}\right]\right)$,

where $W$ is a Gaussian random variable. When the horizon is infinite, it has been conjectured in [3] and proved rigorously [30] that the Lorentz gas is superdiffusive and more precisely that $\left(\kappa_{n}\right)_{n}$ satisfies the CLT with nonstandard normalization in $\sqrt{n \log n}$, i.e.

$\forall A_{i}<B_{i}, \quad \lim _{n \rightarrow+\infty} \mathbb{P}\left(\frac{\kappa_{n}}{\sqrt{n \log n}} \in\left[A_{1}, B_{1}\right] \times\left[A_{2}, B_{2}\right]\right)=\mathbb{P}\left(W \in\left[A_{1}, B_{1}\right] \times\left[A_{2}, B_{2}\right]\right)$,

where $W$ is some Gaussian random variable. These two different behaviours can be heuristically explained by the fact that $\kappa_{1}$ is uniformly bounded when the horizon is finite and is not square integrable when the horizon is infinite, more precisely that

$$
\mathbb{P}\left(\left|\kappa_{1}\right|>N\right) \approx N^{-2}
$$

While [30] focuses on the case where only obstacle (modulo $\mathbb{Z}^{2}$ ) is tangent to a same line, we consider the more general case and establish the following formula for the asymptotic variance matrix of $W$ generalizing [30]:

$$
\left(a_{i, j}\right)_{i, j=1,2}:=\frac{1}{|\partial \bar{Q}|} \sum_{C \in \mathcal{C}} \frac{\mathfrak{d}_{C}^{2}}{\left|w_{C}\right|} w_{C} \otimes w_{C}
$$

where $w \otimes w$ represents the matrix $\left(\begin{array}{cc}w_{1}^{2} & w_{1} w_{2} \\ w_{1} w_{2} & w_{2}^{2}\end{array}\right)$ if $w=\left(w_{1}, w_{2}\right)$ and where $\mathcal{C}$ is the set of different "corridors" distinct modulo $\mathbb{Z}^{2}$ (see picture and begining of Sect. 4 for details) that can be drawn in $Q$; for each corridor $C \in \mathcal{C}, \mathfrak{d}_{C}$ is its width and $w_{C}$ a vector in $\mathbb{Z}^{2}$ in the direction of the corridor with coprime coordinates (Fig. 3). 


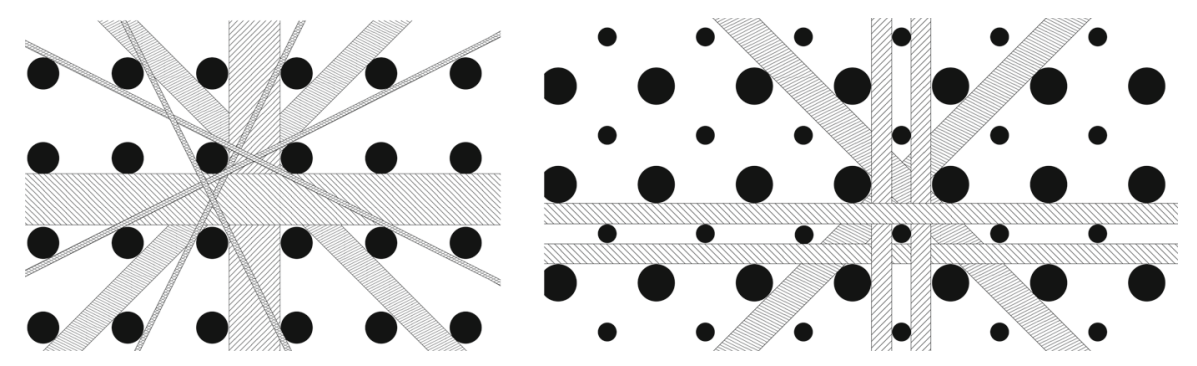

Fig. 3. Corridors for two different periodic billiard domains

We recall the local version of the said CLT, namely LLT gives the asymptotic behaviour of $\mathbb{P}\left(\kappa_{n}=0\right)$, that is of the probability that the particle returns to the initial cell in $\mathbb{Z}^{2}$ at the $n$-th reflection time. Such LLTs have been obtained by Szász and Varjú in [29] in the finite horizon case and in [30] in the infinite horizon case, stating that

$$
\mathbb{P}\left(\kappa_{n}=0\right) \sim \frac{\Phi(0)}{a_{n}^{2}} \text { as } n \rightarrow+\infty,
$$

with $a_{n}=\sqrt{n}$ in the finite horizon case and $a_{n}=\sqrt{n \log n}$ in the infinite horizon case. Here $\Phi$ is the density function of the corresponding Gaussian random variable $W$. Further, [30] uses a version of the local theorem to deduce the recurrence of $\left(X_{n}, \kappa_{n}\right)_{n \geq 1}$. We recall that when the horizon is finite, the recurrence comes directly from the CLT thanks to a general argument due to Conze [8] and Schmidt [27]. When the horizon is finite, a sharp error rate in the LLT has been obtained by Pène [21] and further extensions including expansions of any order in the LLT have been shown in [23]. In this paper, we establish several extensions of the LLT in the infinite horizon case, which is much more delicate due to the lack of finite variance. We emphasize that previous results on LLT [30] and mixing [21] for the infinite measure preserving, infinite horizon Lorentz maps reduce to first order terms.

Tail probability of the first return time to the initial cell (see Theorem 2.1). Let $\tau_{0}$ be the first return time to the initial cell, that is

$$
\tau_{0}:=\min \left\{n \geq 1: \kappa_{n}=(0,0)\right\} .
$$

When the horizon is finite, it was proved in [12] that $\mathbb{P}\left(\tau_{0}>N\right) \sim \frac{1}{\Phi(0) \log N}$. When the horizon is infinite, we show that

$$
\mathbb{P}\left(\tau_{0}>N\right) \sim \frac{1}{\Phi(0) \log \log N} \quad \text { as } \quad N \rightarrow+\infty .
$$

Study of long free flights (see Lemma 4.2). An important ingredient of our proofs for higher order LLT and mixing exploits higher order expansion of $\kappa$. We recall that in the case where only one obstacle (modulo $\mathbb{Z}^{2}$ is tangent to an infinite line contained in the billiard domain), [30, Proposition 6] shows that

$$
\mathbb{P}\left(\kappa_{1}=L+N w\right) \sim \frac{\mathfrak{a}}{|N w|^{3}}+o\left(N^{-3}\right) .
$$


This form was enough in [30] for obtaining the LLT. In our proofs, we need an error of $\mathcal{O}\left(N^{-4}\right)$. Our Lemma 4.2 provides (under an additional regularity assumption) a precise estimate of the following form (with explicit constants $\mathfrak{a}$ and $\mathfrak{a}^{\prime}$ ):

$$
\mathbb{P}\left(\kappa_{1}=L+N w\right)=\frac{\mathfrak{a}}{|N w|^{3}}+\frac{\mathfrak{a}^{\prime}}{|N w|^{4}}+o\left(N^{-4}\right) .
$$

Class of functions. All our results below hold for dynamically Hölder observables and refer to Sect. 2 for precise definition and further assumptions, where required.

Higher order in mixing LLT (see Theorem 2.2 for details). We study a stronger version of the LLT, the mixing LLT (MLLT) which consists in establishing

$$
\mathbb{E}\left[f\left(X_{0}\right) . \mathbf{1}_{\left\{\kappa_{n}=N\right\}} \cdot g\left(X_{n}\right)\right] \sim \frac{\Phi(0) \mathbb{E}\left[f\left(X_{0}\right)\right] \mathbb{E}\left[g\left(X_{0}\right)\right]}{n \log n} \text { as } n \rightarrow+\infty,
$$

when $\mathbb{E}\left[f\left(X_{0}\right)\right] \mathbb{E}\left[g\left(X_{0}\right)\right] \neq 0$. MLLT is about asymptotic independence of $\left(X_{0}, \kappa_{n}, X_{n}\right)$ as $n \rightarrow+\infty$. When $N=0$, we prove in particular that

$$
\mathbb{E}\left[f\left(X_{0}\right) . \mathbf{1}_{\left\{\kappa_{n}=0\right\}} \cdot g\left(X_{n}\right)\right]=\frac{\Phi(0) \mathbb{E}\left[f\left(X_{0}\right)\right] \mathbb{E}\left[g\left(X_{0}\right)\right]}{n \log (n \log n))}+O\left(\frac{1}{n(\log n)^{2}}\right),
$$

with additional error terms detailed in Theorem 2.2. This result ensures in particular that

$$
\mathbb{E}\left[f\left(X_{0}\right) \cdot \mathbf{1}_{\left\{\kappa_{n}=0\right\}} \cdot g\left(X_{n}\right)\right]=\mathbb{E}\left[f\left(X_{0}\right)\right] \mathbb{E}\left[g\left(X_{0}\right)\right] \Phi(0)\left(\frac{1}{n \log n}-\frac{\log \log n}{n(\log n)^{2}}\right)+O\left(\frac{1}{n(\log n)^{2}}\right)
$$

providing a second order term in (6). When $N \neq 0$ is fixed, we obtain an intermediate term ensuring in particular that

$$
\mathbb{E}\left[f\left(X_{0}\right) \cdot \mathbf{1}_{\left\{\kappa_{n}=\lfloor N \sqrt{n \log n}\rfloor\right\}} \cdot g\left(X_{n}\right)\right]=-\frac{\nabla \Phi(N) \cdot \mathfrak{K}(f, g)}{(n \log (n \log n))^{\frac{3}{2}}}+O\left(\frac{1}{(n \log n)^{\frac{3}{2}} \log n}\right)
$$

if $\mathbb{E}\left[f\left(X_{0}\right)\right] \mathbb{E}\left[g\left(X_{0}\right)\right]=0$ with $\mathfrak{K}(f, g)$ a $\mathbb{R}^{2}$-valued bilinear form linearly independent of $\mathbb{E}\left[f\left(X_{0}\right)\right] \mathbb{E}\left[g\left(X_{0}\right)\right]$.

Mixing of general observables in the infinite measure case (Theorem 2.4). The local limit theorem for $\kappa_{n}$ is strongly related to the notion of mixing of dynamical systems preserving an infinite measure, that is the study of the behaviour of quantities of the form

$$
\int_{M} f\left(X_{0}, \kappa_{0}\right) \cdot g\left(X_{n}, \kappa_{n}\right) d \mu
$$

where $\mu$ is the measure absolutely continuous with respect to the Lebesgue measure which is invariant under $\left(X_{n}, \kappa_{n}\right) \mapsto\left(X_{n+1}, \kappa_{n+1}\right)$. A mixing result without error term has been established in [23, Theorems 1.1]. In the present Theorem 2.4 we improve this result to

$$
\int_{M} f\left(X_{0}, \kappa_{0}\right) \cdot g\left(X_{n}, \kappa_{n}\right) d \mu=O\left(n^{-\frac{3}{2}}(\log n)^{-\frac{5}{2}}\right)
$$




$$
+\left(\frac{\Phi(0)}{n \log (n \log n)}+O\left((\log n)^{-1}\right)\right) \int_{M} f\left(X_{0}, \kappa_{0}\right) d \mu \int_{M} g\left(X_{0}, \kappa_{0}\right) d \mu .
$$

In the finite horizon case, an expansion of any order of the form $\int_{M} f\left(X_{0}, \kappa_{0}\right) \cdot g\left(X_{n}, \kappa_{n}\right)$ $d \mu=\sum_{m=1}^{K} \frac{c_{m}(f, g)}{n^{m}}+o\left(n^{-K}\right)$ with $c_{0}(f, g)=\Phi(0) \int_{M} f\left(X_{0}\right) d \mu \int_{M} g\left(X_{0}\right) d \mu$ with $\left(c_{m}(f, g)\right)_{m}$ linearly independent has been established in [23]. Such a result implies, in particular, that for any positive integer $m$, there exist couples of observables $(f, g)$ such that $\int_{M} f\left(X_{0}, \kappa_{0}\right) \cdot g\left(X_{n}, \kappa_{n}\right) d \mu \approx n^{-m}$. In the infinite horizon case, (7) does not imply directly the optimal result for zero mean observables and we address this in a result of independent interest.

Mixing of zero integral observables in the infinite measure case (Theorem 2.5). In the infinite horizon case, we obtain different rates of mixing for null integral functions $f, g$. In particular, we show that

$$
\int_{M} f\left(X_{0}, \kappa_{0}\right) \cdot g\left(X_{n}, \kappa_{n}\right) d \mu \approx(n \log (n \log n))^{-1} n^{-m},
$$

when $g$ and $f$ are coboundaries of respective orders $k, \ell$ with $k+\ell=m$ (a coboundary of order $m$ is a function $g$ of the form $g\left(X_{n}, \kappa_{n}\right)=g_{0}\left(X_{n}, \kappa_{n}\right)-g_{0}\left(X_{n-1}, \kappa_{n-1}\right)$, where $g_{0}$ is a coboundary of order $m-1$, considering here that a function with non null integral is a coboundary of order 0 ) and that

$$
\int_{M} f\left(X_{0}, \kappa_{0}\right) \cdot g\left(X_{n}, \kappa_{n}\right) d \mu \approx(n \log (n \log n))^{-2}
$$

when $f=\phi .\left(\mathbf{1}_{M_{N}}+\mathbf{1}_{M_{-N}}-2 M_{0}\right)$ with $\phi$ and $g$ having non null integral.

Method of proof and main challenges. We heavily exploit that the discrete infinite horizon Lorentz gas is a $\mathbb{Z}^{d}$ extension of the dynamical system $X_{n} \mapsto X_{n+1}$, that is of the form $\left(X_{n}, \kappa_{n}\right) \mapsto\left(X_{n+1}, \kappa_{n+1}=\kappa_{n}+\kappa\left(X_{n}\right)\right)$. We refer to Sect. 2 for further details. We recall that the LLT (without error term) established by Szász and Varjú in [30, Theorem 13] uses the abstract results [2, Theorem 2] of Bálint and Gouëzel, which establishes the non standard CLT for observables of infinite variance (so, not $L^{2}$ ) observables acting on exponential Young towers. The method in [2] was developed to establish the non standard CLT for the stadium billiard, which, among other things, was possible due to the work of Young [32] and Chernov [7]. A classical tool for establishing LLTs for chaotic dynamical systems is the perturbed transfer operator method. As clarified in Sect. 3, a serious challenge for obtaining error terms in the LLT in [30, Theorem 13] is that we need 'sufficiently high' expansions (not just continuity) for the families of eigenvalues and eigenprojectors associated with the transfer operator perturbed with non $L^{2}$ functions. To give a first insight into this difficulty we point out that given the Young Banach space $\mathcal{B}$ (see Sect. 3 for definition), the family of operators is continuous as a family of elements of $\mathcal{L}\left(\mathcal{B} \rightarrow L^{p}\right)$ for some $p>1$ (but not as elements of $\mathcal{L}(\mathcal{B})$ ). Propositions 3.1 and 3.3 provide the expansions for the families of eigenvalues and eigenprojectors we use in the proofs of our main results, already mentioned. The proofs of Propositions 3.1 and 3.3 build on the framework put forward in [2] using several geometrical estimates established in [30]. Under assumptions specific to Young towers for Sinai billiards with infinite horizon, Propositions 3.3 and 3.1 can be viewed as refined version of the main 
technical results in [2]. For a summary of the new ingredients used in the proofs of these propositions we refer to the text after the statement of Proposition 5.3 in Sect. 5.

Let us conclude this introduction with a few remarks on the main examples of infinite measure preserving systems of physical interest. We have already recalled infinite measure preserving periodic Lorentz gases. As already mentioned, LLTs for Sinai billiards can be translated into first order mixing for periodic Lorentz gases. A different type of systems of physical interest are intermittent maps, preserving an infinite measure. To fix notation, we recall a well known interval map, namely the Liverani Saussol Vaienti map [17], $T:[0,1] \rightarrow[0,1], T(x)=x\left(1+2^{\alpha} x^{\alpha}\right)$ if $x \leq 1 / 2$ and $T(x)=2 x-1$ if $x>1 / 2$. Such maps can be viewed as one sided Markov renewal chains with heavy dependencies. We only consider $\alpha \geq 1$, as in this case $T$ is preserving an infinite measure. First order mixing for such maps was obtained by Gouëzel [15] and by Melbourne and Terhesiu [19]. In these works, the $1 / \alpha$-stable LLT (for the first return map of intermittent maps, a much more simple dynamical setting than that of Sinai billiards) is a minor part of the mechanism; in fact, for $\alpha<2$ this type of LLT can be bypassed (see [19]). In short, the mechanisms for obtaining mixing are highly non trivial generalizations of :

(i) The procedure of obtaining the asymptotic of renewal sequences for simple symmetric random walks (in the sense that the LLT is the only required ingredient), in the case of periodic Lorentz gases;

(ii) Proofs of strong renewal theorems (for renewal sequences with infinite mean) for one sided Markov renewal chains, in the case of intermittent maps.

Higher order mixing for (not necessarily Markov) infinite measure preserving intermittent interval maps have been obtained first in [19] and refined in [31]; such results have been generalized to suspension flows in $[4,20]$. We do not know whether results similar to the results on mixing rates for mean zero functions and coboundaries in the setup of Lorentz maps (in [23] and also in Theorem 2.5 therein) hold for infinite measure preserving intermittent interval maps; the previous results $[4,19,20,31]$ for zero integral observables are confined to big $O$ error terms.

Outline of the paper. In Sect. 2, we introduce the precise version of the $\mathbb{Z}^{d}$-periodic billiard model with infinite horizon we consider and state our main results Theorems 2.12 .2 and 2.4. and 2.5. In sect. 3, we present our key technical results Propositions 3.1, 3.3. In Sect. 4, we obtain an expansion for the probability of long free flights, which is crucial for Proposition 3.3. In Sect. 5, we prove our first key result Proposition 3.1, stating an expansion of the dominating eigenprojector. In Sect. 6, we prove our second key result Proposition 3.3, which gives an expansion of the eigenvalue using results contained in the two previous sections. In Sect. 7, we state an expansion in the LLT in a general context and use it to prove our main result as well as a general decorrelation result for some $\mathbb{Z}^{d}$-extensions. Some further technical estimates, as well as the proof of Theorem 2.1, are included in Appendix.

\section{Model and Main Results}

We start by presenting the two dimensional case, that is when $d=2$. We consider a planar billiard domain $Q$ given by $Q:=\mathbb{R}^{2} \backslash \bigcup_{j \in \mathcal{J}, \ell \in \mathbb{Z}^{2}} \mathcal{O}_{j, \ell}$, with $\mathcal{J}$ a non empty finite set and with $\mathcal{O}_{j, \ell}:=\mathcal{O}_{j}+\ell$, where the $\mathcal{O}_{j}$ are open convex set with boundary $C^{3}$ and with nonzero curvature, such that $\mathcal{O}_{j, \ell}$ have pairwise disjoint closures. We assume 


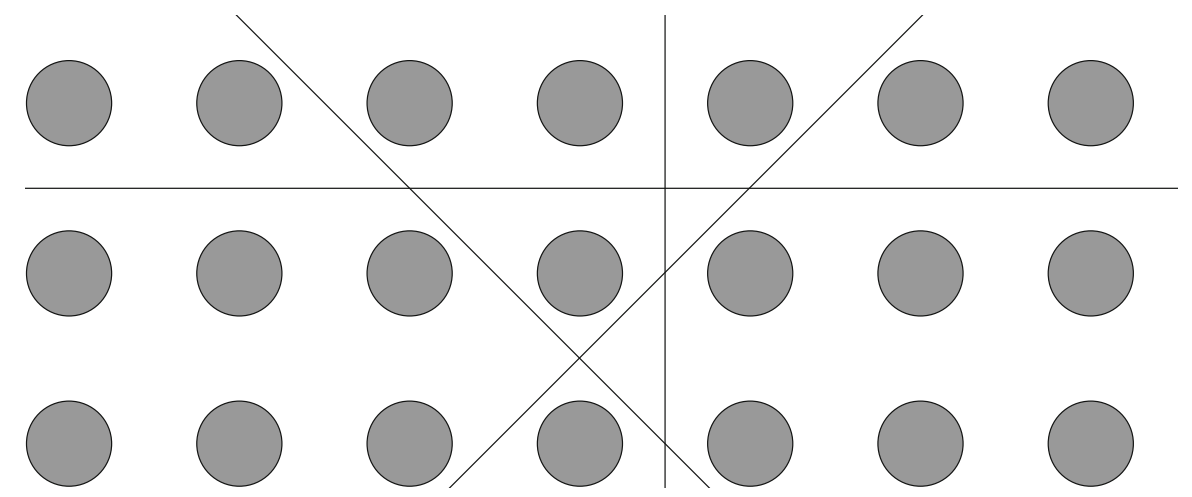

Fig. 4. A periodic billiard domain with 4 infinite horizon directions

that the billiard has infinite horizon, i.e. that $Q$ contains at least one line. When $d=1$, we replace $Q$ and the $\mathcal{O}_{j, \ell}$ by their quotients modulo $\mathbb{Z} \times\{0\}$, that is $Q$ is a subset of the tube $\mathbb{T} \times \mathbb{R}$ (Fig. 4).

We denote by $(M, T, \mu)$ the original billiard dynamical system map corresponding to collision times. The configuration space $M$ is the set of couples of position and velocity $(q, \vec{v})$ with $q \in \partial Q$ and $\vec{v}$ a unit reflected vector, i.e. a unit vector $\vec{v}$ oriented inside $Q$. The billiard map $T$ maps a configuration $(q, \vec{v})$ corresponding to a collision time to the configuration corresponding to the next collision time. The measure $\mu$ is the measure on $M$ with density proportional to $\cos \varphi$, where $\varphi$ is the angle of $\vec{v}$ with the normal vector to $\partial Q$ directed inside $Q$, normalized so that $\mu\left(\left\{(q, \vec{v}) \in M: q \in \bigcup_{j \in \mathcal{J}} \partial \mathcal{O}_{j}\right\}\right)=1$. The infinite measure preserving dynamical system $(M, T, \mu)$ is canonically isomorphic to the $\mathbb{Z}^{d}$-extension of $(\bar{M}, \bar{T}, \bar{\mu})$ by $\kappa: \bar{M} \rightarrow \mathbb{Z}^{d}$, where $(\bar{M}, \bar{T}, \bar{\mu})$ is the probability preserving billiard dynamical system in the billiard domain in $\bar{Q}=Q / \mathbb{Z}^{2} \subset \mathbb{T}^{2}$ if $d=2$ (and $\bar{Q}=Q /(\{0\} \times \mathbb{Z}) \subset \mathbb{T}^{2}$ if $d=1$ ) and $\bar{\mu}$ the probability measure with density proportional to $\cos \varphi$. Let us give the formula of assymptotic variance $\Sigma^{2}$. We take $\Sigma^{2}=\left(a_{i, j}\right)_{i, j=1, \ldots, d}$ with $\left(a_{i, j}\right)_{i, j=1,2}$ given in (2). Note that (2) coincide with the formula of [30, Theorem 20] in the case of a single obstacle since in this case a corridor corresponds to four points $x \in R_{0}$ s.t. $T x=x$ (two positions, one on each side of the corridor, and two directions $\left.\pm \frac{w_{C}}{\left|w_{C}\right|}\right)$. We assume $\Sigma^{2}$ invertible, i.e. the interior of $Q$ contains at least $d$ unbounded lines not parallel to each other (one may observe that when $d=1$ the invertibility of $\Sigma^{2}=a_{1,1}$ just means that $a_{1,1} \neq 0$ ). For any $x \in \mathbb{R}^{d}$, we write $\Phi_{\Sigma^{2}}(x):=\frac{e^{-\frac{1}{2} \Sigma^{-2} x \cdot x}}{\sqrt{(2 \pi)^{d} \operatorname{det} \Sigma^{2}}}, \Phi_{\Sigma^{2}}$ is the density function of a Gaussian distribution with expectation 0 and variance matrix $\Sigma^{2}$. We set $\tau_{0}:=\min \left\{n \geq 1: \kappa_{n}=(0,0)\right\}$ with $\kappa_{n}:=\sum_{k=0}^{n-1} \kappa \circ \bar{T}^{k}$.

Theorem 2.1 (Tail probability of the first return time in the initial cell).

$$
\begin{aligned}
& \text { If } d=2: \quad \bar{\mu}\left(\tau_{0}>N\right) \sim \frac{2 \pi \sqrt{\operatorname{det} \Sigma^{2}}}{\log \log N} \text { as } N \rightarrow+\infty \\
& \text { If } d=1: \bar{\mu}\left(\tau_{0}>N\right) \sim \sqrt{\frac{2 a_{1,1} \log N}{\pi N}} \text { as } N \rightarrow+\infty .
\end{aligned}
$$


Our other main theorems will require the following additional assumption (ensuring (5)):

$\partial Q$ is $C^{4}$ at points $q \in \partial Q$ such that the tangent line to $\partial Q$ at $q$ is contained in $Q$.

Let us introduce the class of smooth functions we consider. Let $R_{0} \subset M$ be the set of reflected vectors that are tangent to $\partial Q$. The billiard map $T$ defines a $C^{1}$-diffeomorphism from $M \backslash\left(R_{0} \cup T^{-1} R_{0}\right)$ onto $M \backslash\left(R_{0} \cup T R_{0}\right)$. For any integers $k \leq k^{\prime}$, we set $\xi_{k}^{k^{\prime}}$ for the partition of $M \backslash \bigcup_{j=k}^{k^{\prime}} T^{-j} R_{0}$ in connected components and $\xi_{k}^{\infty}:=\bigvee_{j \geq k} \xi_{k}^{j}$. For any $\phi: M \rightarrow \mathbb{R}$ and any $\eta \in(0,1)$, we set

$$
L_{\phi, \eta}:=\sup _{k \geq 0} \sup _{A \in \xi_{-k}^{k}} \sup _{x, y \in A} \frac{|\phi(x)-\phi(y)|}{\eta^{k}} \text { and }\|\phi\|_{(\eta)}:=\|\phi\|_{\infty}+L_{\phi, \eta} .
$$

For $\phi: \bar{M} \rightarrow \mathbb{R}$, we define $R_{0}, \xi_{k}^{j}, L_{\phi, \eta},\|\phi\|_{(\eta)}$ in the same way with $(\bar{M}, \bar{T})$ instead of $(M, T)$.

Theorem 2.2 (Mixing local limit theorem). Assume (12). Let $\phi, \psi: \bar{M} \rightarrow \mathbb{R}$ be two measurable functions such that $\|\phi\|_{(\eta)}+\|\psi\|_{(\eta)}<\infty$, then, uniformly in $N \in \mathbb{Z}^{d}$,

$$
\begin{aligned}
& \int_{\bar{M}} \phi 1_{\left\{\kappa_{n}=N\right\}} \psi \circ \bar{T}^{n} d \bar{\mu}=\frac{\mathbb{E}_{\bar{\mu}}[\phi] \mathbb{E}_{\bar{\mu}}[\psi]}{(n \log (n \log n))^{\frac{d}{2}}}\left(\Phi_{\Sigma^{2}}\left(\frac{N}{\sqrt{n \log (n \log n)}}\right)+O\left((\log n)^{-1}\right)\right) \\
& -\nabla \Phi_{\Sigma^{2}}\left(\frac{N}{\sqrt{n \log (n \log n)}}\right) \cdot \frac{\mathfrak{K}(\phi, \psi)}{(n \log (n \log n))^{\frac{d+1}{2}}}+O\left(\frac{1}{(n \log n)^{\frac{d+1}{2}} \log n}\right) .
\end{aligned}
$$

with $\mathfrak{K}(\phi, \psi):=\mathbb{E}_{\bar{\mu}}[\psi] \sum_{j \geq 0} \mathbb{E}_{\bar{\mu}}\left[\kappa \circ \bar{T}^{j} \phi\right]+\mathbb{E}_{\bar{\mu}}[\phi] \sum_{j \leq-1} \mathbb{E}_{\bar{\mu}}\left[\kappa \circ \bar{T}^{j} \psi\right]$, these sums being absolutely convergent, and with $\nabla$ the gradient operator.

Observe that the two first terms of (14) both contain expansions since the first term can be rewritten $\frac{\mathbb{E}_{\bar{\mu}}[\phi] \mathbb{E}_{\bar{\mu}}[\psi]}{(n \log n)^{\frac{d}{2}}}\left(\Phi_{\Sigma^{2}}\left(\frac{N}{\sqrt{n \log n}}\right)\left(1+\left(\frac{\Sigma^{-2} N \cdot N}{n \log n}-d\right) \frac{\log \log n}{2 \log n}\right)+O\left(\frac{1}{\log n}\right)\right)$ and the second one $-\nabla \Phi_{\Sigma^{2}}\left(\frac{N}{\sqrt{n \log n}}\right) \cdot \frac{\mathfrak{K}(\phi, \psi)}{(n \log n)^{\frac{d+1}{2}}}\left(1-\frac{1}{2}\left(d+2-\frac{\Sigma^{-2} N \cdot N}{n \log n}\right) \frac{\log \log n}{\log n}\right)$. The assumption that the interior of $Q$ contains at least $d$ non parallel infinite lines ensures that det $\Sigma^{2} \neq 0$. For any $N \in \mathbb{Z}^{d}$, we write $M_{N}$ for the set of $(q, v) \in M$ such that $q \in \bigcup_{j \in \mathcal{J}} \partial \mathcal{O}_{j, N}$.

Remark 2.3. Observe that the bilinear forms $\mathbb{E}_{\mu}[\phi] \mathbb{E}_{\mu}[\psi]$ and $\mathfrak{K}(\phi, \psi)$ are linearly independent. Indeed, under the assumptions of Theorem 2.2 , then $\mathfrak{K}(\phi-\phi \circ \bar{T}, \psi)=$ $-\mathbb{E}_{\mu}[\psi] \mathbb{E}_{\mu}[\kappa . \phi \circ \bar{T}]$ which is non zero in general.

Theorem 2.4 (Decorrelation in infinite measure). Assume (12). Let $\eta, \gamma \in(0,1)$. Let $\phi, \psi: M \rightarrow \mathbb{R}$ be two measurable observables such that $\sum_{N \in \mathbb{Z}^{2}}(1+$ $\left.|N|^{\gamma}\right)\left(\left\|\phi 1_{M_{N}}\right\|_{\infty}+\left\|\psi 1_{M_{N}}\right\|_{\infty}\right)<\infty$ and $\sum_{N \in \mathbb{Z}^{2}} L_{\phi 1_{M_{N}}, \eta}<\infty$. Then

$$
\int_{M} \phi . \psi \circ T^{n} d \mu=\frac{\Phi_{\Sigma^{2}}(0)}{(n \log (n \log n))^{\frac{d}{2}}} \int_{M} \phi d \mu \int_{M} \psi d \mu+O\left(\frac{1}{(n \log n)^{\frac{d}{2}} \log n}\right) .
$$


If moreover $\sum_{N \in \mathbb{Z}^{2}}\left(1+|N|^{1+\gamma}\right)\left(\left\|\phi 1_{M_{N}}\right\|_{\infty}+\left\|\psi 1_{M_{N}}\right\|_{\infty}\right)<\infty$, then

$$
\begin{aligned}
\int_{M} \phi . \psi \circ T^{n} d \mu= & \frac{\Phi_{\Sigma^{2}}(0)}{(n \log (n \log n))^{\frac{d}{2}}}\left(1+O\left(\frac{1}{\log n}\right)\right) \int_{M} \phi d \mu \int_{M} \psi d \mu \\
& +O\left(\frac{1}{(n \log n)^{\frac{d+1}{2}} \log n}\right) .
\end{aligned}
$$

Again, in the above result, $(n \log (n \log n))^{-\frac{d}{2}}$ can be replaced by $\frac{1-\frac{d}{2} \frac{\log \log n}{\log n}}{(n \log n)^{\frac{d}{2}}}$ providing a second term in $\frac{\log \log n}{(n \log n)^{\frac{d}{2}} \log n}$. When $\phi$ or $\psi$ has zero mean, Theorem 2.4 only provides an estimate in $O(\cdot)$. Luckily, our method enables us to establish sharp decorrelation rates

for zero mean observables under natural regularity assumptions. This includes smooth coboundaries.

Theorem 2.5 (Sharper decorrelation rates for particular functions with zero integral). Assume (12). Let $\gamma \in(0,1)$.

(a) Let $\phi, \psi: M \rightarrow \mathbb{C}$ be observables such that $\sum_{N \in \mathbb{Z}^{d}}\left(1+|N|^{\gamma}\right)\left(\left\|\phi 1_{M_{N}}\right\|_{\infty}+\| \psi 1_{M_{N}}\right.$ $\left.\|_{\infty}\right)<\infty$ and $\sum_{N \in \mathbb{Z}^{d}} L_{\phi 1_{M_{N}}, \eta}<\infty$. Then

$$
\begin{aligned}
\int_{M} \phi . \psi \circ(i d-T)^{m} \circ T^{n} d \mu= & -\frac{\Phi_{\Sigma^{2}}(0) \int_{M} \phi d \mu \int_{M} \psi d \mu}{(n \log (n \log n))^{\frac{d}{2}} n^{m}}(-2)^{-m} d(d+2) \cdots(d+2 m-2) \\
& +O\left((n \log n)^{\left.-\frac{d}{2} n^{-m}(\log n)^{-1}\right)},\right.
\end{aligned}
$$

with $(-2)^{-m} d(d+2) \cdots(d+2 m-2)=(-1)^{m} m$ ! when $d=2$.

(b) Let $N \in \mathbb{Z}^{d}$. If $\phi: M \rightarrow \mathbb{C}$ is invariant by translation of positions by $\mathbb{Z}^{d}$ and satisfies $\|\phi\|_{(\eta)}<\infty$ and if there exists $\delta \in(0,1]$ such that $\sum_{N \in \mathbb{Z}^{d}}\left(\left\|\psi 1_{M_{N}}\right\|_{(\eta)}+N^{\delta}\left\|\psi 1_{M_{N}}\right\|_{\infty}\right)<\infty$, then, setting $f_{0}=\phi\left(1_{M_{N}}+1_{M_{-N}}-\right.$ $\left.2 \times 1_{M_{0}}\right)$,

$$
\begin{aligned}
\int_{M} f_{0} . \psi \circ T^{n} d \mu= & -\frac{\Phi_{\Sigma^{2}}(0) \int_{M_{0}} \phi d \mu \int_{M} \psi d \mu}{(n \log (n \log n))^{\frac{d}{2}+1}}\left(\Sigma^{-2} N \cdot N+O\left((\log n)^{-1}\right)\right) \\
& +O\left(\frac{\log n}{(n \log n)^{\frac{d+3}{2}}}+a_{n}^{-d-2-\delta}\right) .
\end{aligned}
$$

Again $\left((n \log (n \log n))^{-\frac{d}{2}-m}\right.$ in the above formulas can be replaced by $\frac{1-\frac{(d+2 m) \log \log n}{2 \log n}}{(n \log n)^{\frac{d}{2}+m}}$ an expansion with two terms. Let us make several observations on this last result. First, whereas in the finite horizon case, we only have leading terms in $n^{-d / 2-m}$ in the decorrelation of smooth functions, in the infinite horizon case we can have leading terms in $n^{-m}(n \log n)^{-d / 2}$ but also in $(n \log n)^{-d / 2-1}$. Other orders are possible. For example, we can easily adapt our proof to obtain sharp decorrelation rate in $n^{-\frac{d}{2}-m-1}(\log n)^{-d / 2-1}$ in case (b) with $\psi$ a coboundary of order $m$. Observe that, when $m=1$, Case (a) of Theorem 2.5 corresponds to the study of $\int_{M} \phi . \psi \circ T^{n} d \mu-\int_{M} \phi . \psi \circ T^{n-1} d \mu$ and the dominating term given by (a) is equivalent to the difference between the two leading terms of $\int_{M} \phi . \psi \circ T^{n} d \mu$ and of $\int_{M} \phi . \psi \circ T^{n-1} d \mu$ obtained in Theorem 2.4. The leading 
term is of order $(n \log n)^{-d}-((n+1) \log (n+1))^{-d} \sim d(n \log n)^{-\frac{d}{2}-1} \log n$. Observe that the case when $\phi$ is a coboundary and the case of two coboundaries is included in item (a) of theorem 2.5. Indeed, by $T$-invariance of $\mu$,

$$
\begin{aligned}
\int_{M} \phi \circ(i d-T) . \psi \circ T^{n} d \mu & =\int_{M} \phi . \psi \circ T^{n} d \mu-\int_{M} \phi . \psi \circ T^{n-1} d \mu \\
& =-\int_{\Omega} \phi . \psi \circ(i d-T) \circ T^{n-1} d \mu
\end{aligned}
$$

and thus

$\int_{M} \phi \circ(i d-T)^{r} . \psi \circ(i d-T)^{s} \circ T^{n} d \mu=(-1)^{r} \int_{M} \phi . \psi \circ(i d-T)^{r+s} \circ T^{n-r} d \mu$.

Theorems 2.2 and 2.4 are contained in the more technical Theorems 7.6 and 7.7 (valid for a class of less regular observables) which are consequences of Theorem 7.1 that gives higher order terms in LLT and speed of mixing under abstract assumptions on families of eigenvalues and eigenprojectors. Most of our work consist in proving the results contained in the next section enabling the application of Theorem 7.1 to the quotiented tower $(\Delta, f, \mu)$ and $\hat{\kappa}$.

\section{Key Technical Estimates}

We focus on the case $d=2$ since the similar results in the case $d=1$ follow from them. We do not assume here that the interior of $Q$ contains at least $d$ non parallel infinite lines. Our results are based on Fourier analysis and Young towers. It is known that $(\bar{M}, \bar{T}, \bar{\mu})$ is a factor, under a projection written $\mathfrak{p}_{1}: \bar{\Delta} \rightarrow \bar{M}$, of a Young tower $\left(\bar{\Delta}, \bar{f}, \bar{\mu}_{\Delta}\right)$ with stable and unstable curves. By factorizing/collapsing the stable curves we can reduce it to a one-dimensional Young tower $\left(\Delta, f, \mu_{\Delta}\right)$ by $\mathfrak{p}_{2}: \bar{\Delta} \rightarrow \Delta$. Throughout we let $\hat{\kappa}: \Delta \rightarrow \mathbb{Z}^{d}$ be the version of $\kappa$ on $\Delta$, that is $\hat{\kappa} \circ \mathfrak{p}_{2}=\kappa \circ \mathfrak{p}_{1}$ (the existence of such a $\hat{\kappa}$ comes from the fact that $\kappa$ is constant on the stable curves). Let $P$ be the transfer operator for $\left(\Delta, f, \mu_{\Delta}\right)$. We consider the family $\left(P_{t}\right)_{t \in \mathbb{R}}$ of perturbations of $P$ given by $P_{t}:=P\left(e^{i t \cdot \hat{\kappa}} \cdot\right)$, where $\cdot$ denotes the standard scalar product on $\mathbb{R}^{d}$. Note that $P_{0} \equiv P$. As shown in [30] thanks to [7,32] there exist $\beta \in(0, \pi]$ and $\theta \in(0,1)$ such that for every $t \in[-\beta, \beta]^{d}$,

$$
\begin{aligned}
& P_{t}^{n}=\lambda_{t}^{n} \Pi_{t}+N_{t}^{n}, \\
& \text { with } \\
& \sup _{t \in[-\beta, \beta]^{d}}\left\|N_{t}^{n}\right\|_{\mathcal{B}}+\sup _{t \in[-\pi, \pi]^{d} \backslash[-\beta, \beta]^{d}}\left\|P_{t}^{n}\right\|_{\mathcal{B}}=O\left(\theta^{n}\right), \\
& \text { and } \quad \lim _{t \rightarrow 0} \| \Pi_{t}-\mathbb{E}_{\mu_{\Delta}[\cdot] \mathbf{1} \|_{\mathcal{L}\left(\mathcal{B} \rightarrow L^{1}\left(\mu_{\Delta}\right)\right)}=0,}
\end{aligned}
$$

where $\mathcal{B}$ is a complex Banach space of $\mathbb{C}$-valued and $\mu_{\Delta}$-integrable functions (considered by Young in [32]). As in the finite horizon case, $\left(P_{t}\right)_{t}$ defines a family of operators on $\mathcal{B}$. But, whereas in the finite horizon case $t \mapsto P_{t}$ is $C^{\infty}$ from $\mathbb{R}$ to $\mathcal{L}(\mathcal{B})$ the set of linear continuous operators on $\mathcal{B}$, in the infinite horizon case we can just say that $t \mapsto P_{t}$ is continuous from $\mathbb{R}$ to $\mathcal{L}\left(\mathcal{B} \rightarrow L^{1}\left(\mu_{\Delta}\right)\right)$. Additionally the derivative of $P_{t}$ at 0 should be $P(i \hat{\kappa} \cdot)$ which is not in $\mathcal{L}(\mathcal{B})$ not even in $\mathcal{L}\left(L^{1}\right)$ (see Lemma 5.1) but is in $\mathcal{L}\left(L^{p} \rightarrow L^{q}\right)$ as soon as $\frac{1}{p}+\frac{1}{2}>\frac{1}{q}$. As shown in [30, Proposition 6], $\bar{\mu}(\kappa=L+N \vec{w}) \sim C_{L, \vec{w}} N^{-3}(1+o(1))$ for some $C_{L, \vec{w}} \geq 0$, strictly positive for some $L$ 
if there exists a line of direction $\vec{w}$ contained inside $Q$. Combining this with (15), (16), several lemmas obtained inside [30, Proof of Theorem 13] and [2, Proposition 4.17], Szász and Varjú established in [30] the following estimate

$$
\lambda_{t}=1-\Sigma^{2} t \cdot t \log (1 /|t|)(1+o(1)), \quad \text { as } t \rightarrow 0 .
$$

Inside the proof of our Proposition 3.3 below, which gives a higher order expansion of $\lambda_{t}$ at $t=0$, we provide a precise summary of the results in [30] needed to obtain (18). Let us recall that (15), (16), (17) and (18) imply the LLT for $\bar{T}$ and thus first order mixing (speed of mixing) for $T$ as in [23, Theorem 1.1]. Here we are interested in higher order terms in both the LLT and mixing for suitable classes of functions.

Proposition 3.1. There exists a functional Banach space $\mathcal{B}_{0}$ of bounded functions and $\Pi_{0}^{\prime} \in \mathcal{L}\left(\mathcal{B}_{0} \rightarrow L^{s}\left(\mu_{\Delta}\right)\right)$ for every $s \in[1,2)$ such that, for any $p>2$, any $p^{\prime} \in$ $\left[1, \frac{4}{3}\right)$, any $\gamma \in\left(1, \min \left(2 \frac{p-1}{p}, \frac{4}{p^{\prime}}-2\right)\right)$, there exists $C>0$ such that $\|\left(\Pi_{t}-\Pi_{0}-t\right.$. $\left.\Pi_{0}^{\prime}\right)(w) \|_{\mathcal{L}\left(\mathcal{B}_{0} \rightarrow L^{p^{\prime}}\left(\mu_{\Delta}\right)\right)} \leq C|t|^{\gamma}$ as $t \rightarrow 0$.

Proposition 3.1 follows directly from Proposition 5.3 by taking $v=1_{\Delta}$.

Remark 3.2. A precise formula for $\Pi_{0}^{\prime}$ is given by (33) of Proposition 5.3 together with (36) of Proposition 5.4.

Proposition 3.3. Assume (12). As $t \rightarrow 0, \lambda_{t}=1-\Sigma^{2} t \cdot t \log \left(|t|^{-1}\right)+O\left(t^{2}\right)$ with the notations introduced at the begining of Sect. 4.

Since the norms of $\mathbb{R}^{d}$ are all metrically equivalent, Proposition 3.3 is true for any choice of norm $|\cdot|$ on $\mathbb{R}^{d}$.

\section{Estimate of the Probability of Long Free Flights}

Our proof of Proposition 3.3 provided in Sect. 6 is based on estimates established in Sect. 5 and on an higher order expansion of the tail of the free flight $\kappa$. Lemma 4.2 below gives the required expansion (to be precise we just use $O\left(N^{-4}\right)$ ). We mention that Lemma 4.2 can be regarded as a refinement of [30, Proposition 6]. First, it provides higher order of the tail of the free flight and second, we compute the dominating term without any restriction on the number of types of scatterer at the boundary of a given corridor. As in [30], we introduce the notion of corridor. We call corridor $C$ a strip contained in $Q$ delimited by $(A+\mathbb{R} w) \cup(B+L+\mathbb{R} w)$ for some $A, B \in \bigcup_{j \in \mathcal{J}} \partial \mathcal{O}_{j}$ and $L, w \in \mathbb{Z}^{2}$. We write $\mathcal{C}$ for the set of corridors. For any corridor $C \in \mathcal{C}$, we write $\mathfrak{d}_{C}$ for its width and $w_{C}$ for its direction that is the prime $w \in \mathbb{Z}^{2} \backslash\{0\}$ with non negative first coordinate integer coordinates (and with positive second coordinate if the first one is null) such that the $C$ contains a line of direction $w_{C}$ (Fig. 5).

Observe that a corridor is fully determined by $A$ and that, given $A$ defining a corridor, there are two possible choices of prime $w \in \mathbb{Z}^{2}$, one being the opposite of the other. We write $\mathcal{A}$ for the finite set of $(A, w) \in\left(\bigcup_{j \in \mathcal{J}} \partial \mathcal{O}_{j}\right) \times \mathbb{Z}^{2}$, with $w$ prime, such that there exists $(B, L)$ as above and $d_{A}$ for the width of the corresponding corridor.

Remark 4.1. Let $(A, w) \in \mathcal{A}$ then $(A, v=w /|w|) \in \bigcap_{k \in \mathbb{Z}} T^{k}\left(R_{0}\right)$. With the above notations, let $\mathcal{O}$ be the obstacle containing $B+L$. If $\left(q^{\prime}, v^{\prime}\right) \in \bigcap_{k \in \mathbb{Z}} T^{k}\left(R_{0}\right) \backslash\{(A, v)\}$ is close to $(A, v)$, the line $q^{\prime}+\mathbb{R} v^{\prime}$ passes between $\mathcal{O}+M w$ and $\mathcal{O}+(M+1) w$ for some $M \in \mathbb{Z}$ without passing through them. But, this is impossible if $\left|\angle\left(v, v^{\prime}\right)\right|<\arctan \frac{2 r}{|w|}$ with $r$ the minimal radius of curvature of $\mathcal{O})$. Thus $(A, v)$ is isolated in $\bigcap_{k \in \mathbb{Z}} T^{k}\left(R_{0}\right)$. Therefore $\mathcal{A}$ is finite. 


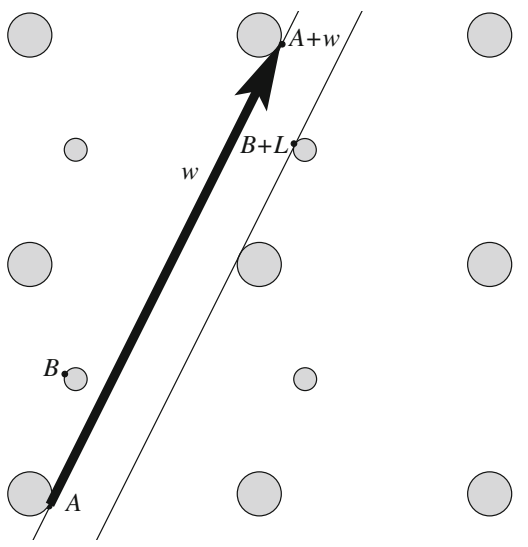

Fig. 5. A corridor

Given $L, w \in \mathbb{Z}^{2}$, we write $E_{L, w}$ for the set of $(A, B)$ such that $(A, w) \in \mathcal{A}$ and $B \in \partial Q$ is as described above (on the other line delimiting the corridor).

Lemma 4.2. Assume (12). Let $w \in \mathbb{Z}^{2}$ prime and $L \in \mathbb{Z}^{2}$, then

$$
\bar{\mu}(\kappa=L+N w)=\sum_{(A, B) \in E_{L, w}}\left(\frac{d_{A}^{2} \mathfrak{a}_{(A, B)}}{2|\partial \bar{Q}||w| N^{3}}+\frac{\mathfrak{a}_{(A, B)}^{\prime}}{2|\partial \bar{Q}|(|w| N)^{4}}\right)+o\left(N^{-4}\right),
$$

with $\mathfrak{a}_{(A, B)}:=\frac{A A^{\prime} B^{\prime \prime} B}{|w|^{2}}$ and

$\mathfrak{a}_{(A, B)}^{\prime}:=\frac{3 d_{A}^{2} A A^{\prime} B^{\prime \prime} B\left(A A^{\prime}+B^{\prime \prime} B-2 \frac{w \cdot \overrightarrow{A(B+L)}}{|w|}\right)}{2}+\frac{d_{A}^{3}}{2}\left(B^{\prime \prime} B\left(\frac{1}{\mathfrak{c}_{A}}-\frac{1}{\mathfrak{c}_{A^{\prime}}}\right)+A A^{\prime}\left(\frac{1}{\mathfrak{c}_{B}}-\frac{1}{\mathfrak{c}_{B^{\prime \prime}}}\right)\right)$.

where $B^{\prime \prime}$ is the first point of $\partial Q$ met by the half-line $B-\mathbb{R}_{+} w$ and where $A$ ' is the first point of $\partial Q$ met by the half-line $A+\mathbb{R}_{+} w$ and where $\mathfrak{c}_{E}$ is the curvature of $\partial Q$ at any $E \in \partial Q$.

For example, in the case of a billiard with a single original obstacle (if $\mathcal{J}=1$ ), then $\bar{\mu}(\kappa=(N, 1))=\frac{d^{2}}{2|\partial \bar{Q}|}\left(N^{-3}+3(1-h) N^{-4}+o\left(N^{-4}\right)\right)$ (then $A=A^{\prime}, B=B^{\prime \prime}$ and so $\frac{1}{\mathfrak{c}_{A}}-\frac{1}{\mathfrak{c}_{A^{\prime}}}=\frac{1}{\mathfrak{c}_{B}}-\frac{1}{\mathfrak{c}_{B^{\prime \prime}}}=0$ ), where $d$ is the vertical distance between two different obstacles and $h$ is the horizontal gap between the highest point and the lowest point of the obstacle ( $h$ can be negative or positive) (Fig. 6).

Corollary 4.3. Assume (12). Let us fix a corridor $C$. The sum $\mathfrak{a}_{C}$ of the $\mathfrak{a}_{(A, B)}$ over the couples $(A, B)$ associated to $C$ is 2 .

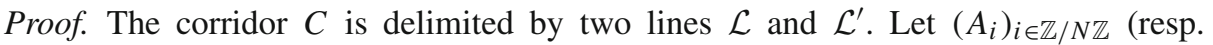
$\left.\left(B_{j}\right)_{j \in \mathbb{Z} / N^{\prime} \mathbb{Z}}\right)$ be the points of $\bigcup_{j=1}^{\mathcal{J}} \partial \mathcal{O}_{j} \cap\left(\mathcal{L}+\mathbb{Z}^{2}\right)\left(\right.$ resp. $\left.\bigcup_{j=1}^{\mathcal{J}} \partial \mathcal{O}_{j} \cap\left(\mathcal{L}^{\prime}+\mathbb{Z}^{2}\right)\right)$ ordered so that $A_{i}^{\prime}=A_{i+1}+\ell_{i}$ and $B_{j}^{\prime \prime}=B_{j-1}+\ell_{j}^{\prime}$ for some $\ell_{i}, \ell_{j}^{\prime} \in \mathbb{Z}^{2}$. Then $\sum_{i} \overrightarrow{A_{i} A_{i}^{\prime}}=\sum_{j} \overrightarrow{B_{j}^{\prime \prime} B_{j}}=w_{C}$, and so 


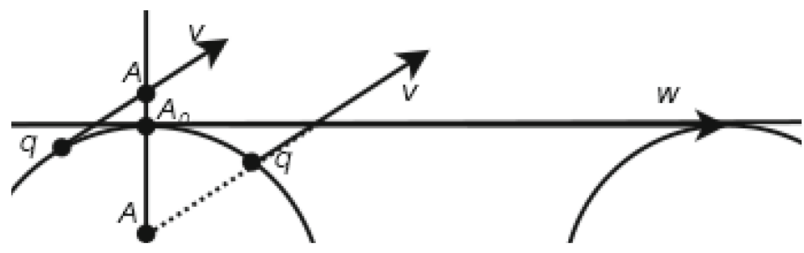

Fig. 6. Projected point $A$

$$
\mathfrak{a}_{C}=\sum_{i, j}\left(\mathfrak{a}_{\left(A_{i}, B_{j}\right)}+\mathfrak{a}_{\left(B_{j}, A_{i}\right)}\right)=\sum_{i, j} \frac{A_{i} A_{i}^{\prime} B_{j}^{\prime \prime} B_{j}+B_{j} B_{j}^{\prime} A_{i}^{\prime \prime} A_{i}}{\left|w_{C}\right|^{2}}=2 .
$$

Proof of Lemma 4.2. Observe that, for every $\varepsilon$, outside the $\varepsilon$-neighbourhood of $\bigcap_{k \in \mathbb{Z}} T^{k}\left(R_{0}\right), \kappa$ is uniformly bounded. Moreover, outside the $\varepsilon$-neighbourhood of points $\left(A_{0}, w /|w|\right)$ with $\left(A_{0}, B\right) \in E_{L, w}$, for some $N_{0}$ large enough, $\kappa \notin L+\left(N_{0}+\mathbb{N}\right) w$. Therefore, it is enough to prove that, for any $\left(A_{0}, B\right) \in E_{L, w}$ and any $i_{0}, i_{1} \in \mathcal{J}$ so that $A_{0} \in \partial \mathcal{O}_{i_{0}}$ and $B \in \partial \mathcal{O}_{i_{1}}$,

$$
\mu\left(M_{\left(i_{0}, 0\right)} \cap T^{-1}\left(M_{\left(i_{1}, L+N w\right)}\right)\right)=\frac{d_{0}^{2} \mathfrak{a}_{\left(A_{0}, B\right)}}{2|\partial \bar{Q}||w| N^{3}}+\frac{\mathfrak{a}_{\left(A_{0}, B\right)}^{\prime}}{2|\partial \bar{Q}| N^{4}}+o\left(N^{-4}\right),
$$

where $d_{0}:=d_{A_{0}}$ is the width of the corresponding corridor and $M_{(i, \ell)}$ the set of elements of $M$ based on a point in $\partial \mathcal{O}_{i, \ell}$. Set $B_{0}:=B+L$. Let $\mathcal{A}_{N}$ be the image set of $M_{\left(i_{0}, 0\right)} \cap$ $T^{-1}\left(M_{\left(i_{1}, \ell_{1}+N w\right)}\right)$ by the projection map $(q, \vec{v}) \mapsto(A, \vec{v})$ where $A$ is the intersection point of $q+\mathbb{R} \vec{v}$ with the line $A_{0}+\mathbb{R} w^{\perp}$, where $w^{\perp}$ is the unit vector perpendicular to $\vec{w}$ directed inside $Q$ at $A_{0}$. Because of the invariance of $\bar{\mu}$ under the billiard map and under the inversion $(q, \vec{v})=(q,-\vec{v})$, thus, using also coordinates $(x, \alpha)$ on $\left(A_{0}+\mathbb{R} w^{\perp}\right) \times S^{1}$ with $x$ the absciss on the line $A_{0}+\mathbb{R} w^{\perp}$ and $\alpha$ for angle between $w$ and $\vec{v}$, we know that

$$
\mu\left(M_{\left(i_{0}, 0\right)} \cap T^{-1}\left(M_{\left(i_{1}, \ell_{1}+N w\right)}\right)=\frac{1}{2|\partial \bar{Q}|} \int_{\mathcal{A}_{N}} \cos \alpha d x d \alpha .\right.
$$

We write $\mathcal{O}^{\prime}$ for the first obstacle touched by $A_{0}+\mathbb{R}_{+} w\left(\right.$ at $\left.A_{0}^{\prime} \in \partial \mathcal{O}^{\prime}\right)$ and $\mathcal{O}^{\prime \prime}$ for the first obstacle touched by $B_{0}-\mathbb{R}_{+} w$ (at $B^{\prime \prime} \in \partial \mathcal{O}^{\prime \prime}$ ). Let us write $\vec{v}_{\alpha}$ for the unit vector making angle $\alpha$ with $w$, for $\alpha \in[0, \pi / 2]$ and we consider $x$ close to 0 . First, for a given position $A=A_{0}+x w^{\perp}$ close to $A_{0}$ with $x<0,\left(A, \vec{v}_{\alpha}\right)$ is in $\mathcal{A}_{N}$ if and only if the first obstacle (other than $\mathcal{O}_{\left(i_{0}, 0\right)}$ ) met by the half-line $A+\vec{v}_{\alpha} \mathbb{R}_{+}$is $\mathcal{O}_{\left(i_{1}, \ell_{1}+N w\right)}$, that is if and only if $\max \left(\beta, \alpha_{N}\right) \leq \alpha<\alpha_{N}^{\prime}$. where we write $\beta$ for the angle such that the line $A+\vec{v}_{\beta} \mathbb{R}$ is tangent to $\mathcal{O}^{\prime}$ from above, $\alpha_{N}$ for the angle such that the line $q+\vec{v}_{\alpha_{N}} \mathbb{R}$ is tangent to $\mathcal{O}_{\left(i_{1}, \ell_{1}+N w\right)}$ from underneath (at some point $B_{N}^{\prime}$ ) and $\alpha_{N}^{\prime}$ for the angle such that the line $q+\vec{v}_{\alpha_{N}^{\prime}} \mathbb{R}$ is tangent to $\mathcal{O}^{\prime \prime}+N w$ from underneath (at some point $\left.B_{N}^{\prime \prime}\right)($ Fig. 7).

Second, for a given position $A=A_{0}+x w^{\perp}$ close to $A_{0}$ with $x>0,\left(A, \vec{v}_{\alpha}\right)$ is in $\mathcal{A}_{N}$ if and only if the first obstacle met by the half-line $A+\vec{v}_{\alpha} \mathbb{R}_{+}$is $\mathcal{O}_{\left(i_{1}, \ell_{1}+N w\right)}$ and

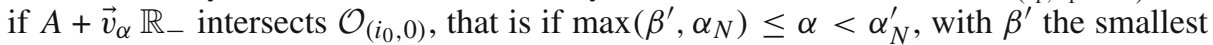
positive angle such that $A+\vec{v}_{\beta^{\prime}}, \mathbb{R}$ is tangent to $\partial \mathcal{O}_{i_{0}}$. Thus, the integral (20) becomes

$$
\frac{I_{N}^{-}+I_{N}^{+}}{2|\partial \bar{Q}|}:=\frac{1}{2|\partial \bar{Q}|}\left(\int_{x^{-}}^{0} \int_{\max \left(\beta, \alpha_{N}\right)}^{\alpha_{N}^{\prime}} \cos \alpha d \alpha d x+\int_{0}^{x^{+}} \int_{\max \left(\beta^{\prime}, \alpha_{N}\right)}^{\alpha_{N}^{\prime}} \cos \alpha d \alpha d x\right) .
$$




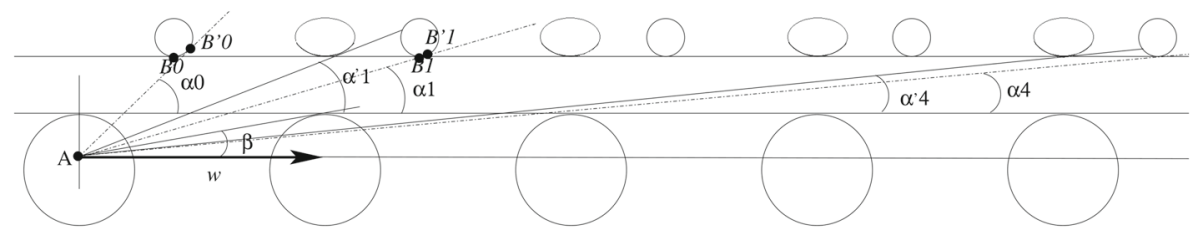

Fig. 7. The $\alpha_{i}$ and $\alpha_{i}^{\prime}$ in a corridor

The proof continues now by giving precise estimates of $\beta, \beta^{\prime}$ and $\alpha_{N}, \alpha_{N}^{\prime}$ and also of the numbers $x^{-}<0<x^{+}$beyond which there are no solutions $\alpha$ to respectively $\max \left(\beta, \alpha_{N}\right) \leq \alpha<\alpha_{N}^{\prime}$ and $\max \left(\beta^{\prime}, \alpha_{N}\right) \leq \alpha<\alpha_{N}^{\prime}$ anymore.

Estimateof $\beta$. Let $q(u)=A_{0}^{\prime}-q_{1}(u) \frac{w}{|w|}-q_{2}(u) w^{\perp}$ be the parametrization of $\partial \mathcal{O}^{\prime}$ by arc-length such that $q(0)=A_{0}^{\prime}$ (i.e. $\left.\left(q_{1}(0), q_{2}(0)\right)=(0,0)\right)$ and $A \in q(s)+\mathbb{R} q^{\prime}(s)$. Observe that $\left(q_{1}^{\prime}(0), q_{2}^{\prime}(0)\right)=(1,0)$ and $\left(q_{1}^{\prime \prime}(0), q_{2}^{\prime \prime}(0)\right)=\left(0, \mathfrak{c}_{A_{0}^{\prime}}\right)$ where $\mathfrak{c}_{A_{0}^{\prime}}$ is the curvature of $\partial \mathcal{O}^{\prime}$ at $A_{0}^{\prime}$. Then $\tan \beta=\frac{q_{2}^{\prime}(s)}{q_{1}^{\prime}(s)}=\frac{\mathfrak{c}_{A_{0}^{\prime}} s+\frac{q_{2}^{\prime \prime \prime}(0)}{2} s^{2}}{1+\frac{q_{1}^{\prime \prime}(0)}{2} s^{2}}+O\left(s^{3}\right)$. So $\beta=\mathfrak{c}_{A_{0}^{\prime}} s+$ $\frac{q_{2}^{\prime \prime \prime}(0)}{2} s^{2}+O\left(s^{3}\right)$, with $s$ such that

$A A_{0}=q_{2}(s)+\left(A_{0} A_{0}^{\prime}-q_{1}(s)\right) \frac{q_{2}^{\prime}(s)}{q_{1}^{\prime}(s)}=\frac{\mathfrak{c}_{A_{0}^{\prime}}}{2} s^{2}+\left(A_{0} A_{0}^{\prime}-s\right)\left(\mathfrak{c}_{A_{0}^{\prime}} s+\frac{q_{2}^{\prime \prime \prime}(0)}{2} s^{2}\right)+O\left(s^{3}\right)$ Thus $s=\frac{A A_{0}}{A_{0} A_{0}^{\prime} \mathfrak{c}_{A_{0}^{\prime}}^{\prime}}\left(1-\frac{1}{2}\left(\frac{q_{2}^{\prime \prime \prime}(0)}{\mathfrak{c}_{A_{0}^{\prime}}}-\frac{1}{A_{0} A_{0}^{\prime}}\right) \frac{A A_{0}}{A_{0} A_{0}^{\prime} \mathfrak{c}_{A_{0}^{\prime}}}\right)+O\left(\left(A A_{0}\right)^{3}\right)$. It follows that

$$
\beta=\frac{A A_{0}}{A_{0} A_{0}^{\prime}}+\frac{\left(A A_{0}\right)^{2}}{2\left(A_{0} A_{0}^{\prime}\right)^{3} \mathfrak{c}_{A_{0}^{\prime}}}+O\left(\left(A A_{0}\right)^{3}\right) .
$$

Estimateof $\beta^{\prime}$. We proceed analogously for $\beta^{\prime}$. This time we consider that $q(u)=A_{0}^{\prime}-$ $q_{1}(u) \frac{w}{|w|}-q_{2}(u) w^{\perp}$ is the parametrization of $\partial \mathcal{O}_{\left(i_{0}, 0\right)}$ by arclength such that $q(0)=A_{0}$ (i.e. $\left.\left(q_{1}(0), q_{2}(0)\right)=(0,0)\right)$ and $A \in q(s)+\mathbb{R} q^{\prime}(s)$. Note that $\left(q_{1}^{\prime}(0), q_{2}^{\prime}(0)\right)=(1,0)$ and $\left(q_{1}^{\prime \prime}(0), q_{2}^{\prime \prime}(0)\right)=\left(0, \mathfrak{c}_{A_{0}^{\prime}}\right)$ where where $\mathfrak{c}_{A_{0}^{\prime}}$ denotes the curvature of $\partial \mathcal{O}^{\prime}$ at $A_{0}^{\prime}$. Then $\tan \beta^{\prime}=\frac{q_{2}^{\prime}(s)}{q_{1}^{\prime}(s)}=\mathfrak{c}_{A_{0}} s+O\left(s^{2}\right)$, with $s$ such that $-A A_{0}=q_{2}(s)-q_{1}(s) q_{2}^{\prime}(s)=$ $-\frac{\mathfrak{c}_{A_{0}}}{2} s^{2}+\mathcal{O}\left(s^{3}\right)$ and so

$$
\beta^{\prime}=\sqrt{2 c_{A_{0}} A A_{0}}+O\left(A A_{0}\right) .
$$

Estimatesof $\alpha_{N}$ andof $\alpha_{N}^{\prime}$. Note that $\alpha_{N}^{\prime}$ is $\alpha_{N+m_{1}}$ for another choice of point $B_{0}$ and for some $m_{1} \in \mathbb{Z}^{2}$ (depending on $B_{1}$ ). Thus it is enough to estimate $\alpha_{N}$. The notation $O(\ldots)$ below will be uniform in $A$. Let $B_{N}:=B_{0}+N w$. We parametrize $\partial \mathcal{O}_{\left(i_{1}, \ell_{1}+N w\right)}$ by arc-length as $q(u)=B_{N}+q_{1}(u) \frac{w}{|w|}+q_{2}(u) w^{\perp}$ such that $q(0)=B_{N}$, $q(s)=B_{N}^{\prime}, q^{\prime}(0)=\frac{w}{|w|}$ and $q^{\prime \prime}(0)=\mathfrak{c}_{B_{0}} w^{\perp}$ where $\mathfrak{c}_{B_{0}}$ is the curvature of $\partial \mathcal{O}_{\left(i_{1}, \ell_{1}\right)}$ at $B_{0}$. Since $\left\langle q^{\prime}(s), q^{\prime}(s)\right\rangle=1,\left\langle q^{\prime \prime}(s), q^{\prime}(s)\right\rangle=0,0=\left\langle q^{\prime \prime \prime}(0), q^{\prime}(0)\right\rangle+\left\langle q^{\prime \prime}(0), q^{\prime \prime}(0)\right\rangle=$ $q_{1}^{\prime \prime \prime}(0)+\mathfrak{c}_{B_{0}}^{2}$ and so $q_{1}^{\prime \prime \prime}(0)=-\mathfrak{c}_{B_{0}}^{2}$. Then $B_{N} B_{N}^{\prime}=|q(s)-q(0)|=s-\frac{\mathfrak{c}_{B_{0}}^{2}}{24} s^{3}+o\left(s^{3}\right)$, 
and so

$$
\begin{aligned}
\tan \alpha_{N} & =\frac{q_{2}^{\prime}(s)}{q_{1}^{\prime}(s)}=\frac{\left.\mathfrak{c}_{B_{0}} s+\frac{1}{2} q_{2}^{\prime \prime \prime}(0) s^{2}+\frac{1}{6} q_{2}^{\prime \prime \prime \prime}(0) s^{3}+o\left(s^{3}\right)\right)}{1+\frac{1}{2} q_{1}^{\prime \prime \prime}(0) s^{2}+o\left(s^{2}\right)} \\
& =\mathfrak{c}_{B_{0}} B_{N} B_{N}^{\prime}+\frac{q_{2}^{\prime \prime \prime}(0)}{2}\left(B_{N} B_{N}^{\prime}\right)^{2}+\mathfrak{b}_{B_{0}}\left(B_{N} B_{N}^{\prime}\right)^{3}+o\left(\left(B_{N} B_{N}^{\prime}\right)^{3}\right),
\end{aligned}
$$

with $\mathfrak{b}_{B_{0}}:=\frac{q_{2}^{\prime \prime \prime \prime}(0)}{6}+\frac{13 \mathfrak{c}_{B_{0}}^{3}}{24}$. Also

$$
\tan \alpha_{N}=\tan \left(T_{B_{N}} \partial \mathcal{O}_{\left(i_{1}, \ell_{1}+N w\right)}, \overrightarrow{A B_{N}^{\prime}}\right)=\frac{w^{\perp} \cdot \overrightarrow{A B_{N}^{\prime}}}{w \cdot \overrightarrow{A B_{N}^{\prime}} /|w|}=\frac{d_{0}^{\prime}(A)+w^{\perp} \cdot \overrightarrow{B_{N} B_{N}^{\prime}}}{|w| N+\frac{w \cdot\left(\overrightarrow{A_{0} B_{0}}+\overrightarrow{B_{N} B_{N}^{\prime}}\right)}{|w|}},
$$

with $d_{0}^{\prime}(A):=w^{\perp} \cdot \overrightarrow{A B_{0}}=d_{0}-w^{\perp} \cdot \overrightarrow{A_{0} A}$. Thus $\alpha_{N}=O\left(N^{-1}\right), B_{N} B_{N}^{\prime}=O\left(N^{-1}\right)$, $A_{0} A=O\left(N^{-1}\right)$. Since $B_{N} \in \partial \mathcal{O}_{\left(i_{1}, \ell_{1}+N w\right)}$ and since $T_{B_{N}} \partial \mathcal{O}_{\left(i_{1}, \ell_{1}+N w\right)}=B_{N}+\mathbb{R} w$, it follows that $w^{\perp} \cdot \overrightarrow{B_{N} B_{N}^{\prime}}=q_{2}(s)=\frac{1}{2} \mathfrak{c}_{B_{0}}\left(B_{N} B_{N}^{\prime}\right)^{2}+o\left(N^{-2}\right)$ and $w \cdot \overrightarrow{B_{N} B_{N}^{\prime}}=$ $|w| B_{N} B_{N}^{\prime}+o\left(N^{-2}\right)$. So

$$
\tan \alpha_{N}=\frac{d_{0}^{\prime}(A)}{\frac{w \cdot \overrightarrow{A_{0} B_{N}}}{|w|}}+\frac{1}{2} \frac{\mathfrak{c}_{B_{0}}\left(B_{N} B_{N}^{\prime}\right)^{2}}{\frac{w \cdot \overrightarrow{A_{0} B_{N}}}{|w|}}-\frac{d_{0}^{\prime}(A) B_{N} B_{N}^{\prime}}{\left(\frac{w \cdot \overrightarrow{A_{0} B_{N}}}{|w|}\right)^{2}}+o\left(N^{-3}\right) .
$$

Identifying (24) with (25), we obtain that

$$
B_{N} B_{N}^{\prime}=\frac{d_{1}}{\frac{w \cdot \overrightarrow{A_{0} B_{N}}}{|w|}}+\frac{d_{2}}{\left(\frac{w \cdot \overrightarrow{A_{0} B_{N}}}{|w|}\right)^{2}}+\frac{d_{3}}{\left(\frac{w \cdot \overrightarrow{A_{0} B_{N}}}{|w|}\right)^{3}}+o\left(N^{-3}\right),
$$

with $d_{1}:=\frac{d_{0}^{\prime}(A)}{\mathfrak{c}_{B_{0}}}, d_{2}:=-\frac{q_{2}^{\prime \prime \prime}(0) d_{1}^{2}}{2 \mathfrak{c}_{B_{0}}}, d_{3}:=-\frac{d_{1}^{2}}{2}-q_{2}^{\prime \prime \prime}(0) \frac{d_{1} d_{2}}{\mathfrak{c}_{B_{0}}}-\mathfrak{b}_{B_{0}} \frac{d_{1}^{3}}{\mathfrak{c}_{B_{0}}}$. Using (24) and $\arctan u=u-\frac{u^{3}}{3}$, it follows that

$$
\alpha_{N}=\frac{d_{0}^{\prime}(A)}{\frac{w \cdot \overrightarrow{A_{0} B_{N}}}{|w|}}-\frac{\frac{\left(d_{0}^{\prime}(A)\right)^{2}}{2 \mathfrak{c}_{B_{0}}}+\frac{\left(d_{0}^{\prime}(A)\right)^{3}}{3}}{\left(\frac{w \cdot \overrightarrow{A_{0} B_{N}}}{|w|}\right)^{3}}+o\left(N^{-3}\right) .
$$

Let us recall that $d_{0}^{\prime}(A):=w^{\perp} \cdot \overrightarrow{A B_{0}}$. Therefore, we also have

$$
\alpha_{N}^{\prime}=\frac{d_{0}^{\prime}(A)}{\frac{w \cdot \overrightarrow{A_{0} B_{N}^{\prime \prime}}}{|w|}}-\frac{\frac{\left(d_{0}^{\prime}(A)\right)^{2}}{2 \mathfrak{c}_{B^{\prime \prime}}}+\frac{\left(d_{0}^{\prime}(A)\right)^{3}}{3}}{\left(\frac{w \cdot \overrightarrow{A_{0} B_{N}^{\prime \prime}}}{|w|}\right)^{3}}+o\left(N^{-3}\right) .
$$

Hence

$$
\alpha_{N}^{\prime}-\alpha_{N}=d_{0}^{\prime}(A)\left(\frac{1}{\frac{w \cdot \overrightarrow{A_{0} B_{N}^{\prime \prime}}}{|w|}}-\frac{1}{\frac{w \cdot \overrightarrow{A_{0} B_{N}}}{|w|}}\right)+\frac{d_{0}^{2}}{2\left(\frac{w \cdot \overrightarrow{A_{0} B_{N}^{\prime \prime}}}{|w|}\right)^{3}}\left(\frac{1}{\mathfrak{c}_{B_{0}}}-\frac{1}{\mathfrak{c}_{B^{\prime \prime}}}\right)+o\left(N^{-3}\right) .
$$


Conclusion Observe that since $\alpha_{N}=O\left(N^{-1}\right)$ and $\alpha_{N}^{\prime}-\alpha_{N}=O\left(N^{-2}\right)$,

$\int_{\max \left(\beta^{\prime \prime}, \alpha_{N}\right)}^{\alpha_{N}^{\prime}} \cos (\varphi) d \varphi=\sin \alpha_{N}^{\prime}-\sin \max \left(\beta^{\prime \prime}, \alpha_{N}\right)=\alpha_{N}^{\prime}-\max \left(\beta^{\prime \prime}, \alpha_{N}\right)+O\left(N^{-4}\right)$, for $\beta^{\prime \prime} \in\left\{\beta, \beta^{\prime}\right\}$. Recalling (21), since $A A_{0}=O\left(N^{-1}\right)$, it follows that

$$
I_{N}^{+}+I_{N}^{-}=O\left(N^{-5}\right)+\int_{0}^{x^{+}} \max \left(0, \alpha_{N}^{\prime}-\max \left(\beta^{\prime}, \alpha_{N}\right)\right) d x+\int_{x^{-}}^{0} \max \left(0, \alpha_{N}^{\prime}-\max \left(\beta, \alpha_{N}\right)\right) d x .
$$

Due (27) and (23) and since $A_{0} A=O\left(N^{-1)}\right.$, for $N$ large enough, $\beta^{\prime}<\alpha_{N}^{\prime}$ if and only if

$$
\sqrt{2 \mathfrak{c}_{A_{0}} A A_{0}}+O\left(A A_{0}\right)<\frac{d_{0}}{|w| N}+O\left(N^{-2}\right), \text { i.e. } A A_{0}<\frac{d_{0}^{2}}{2 \mathfrak{c}_{A_{0}}|w|^{2} N^{2}}+O\left(N^{-3}\right) .
$$

Analogously the condition $\alpha_{N}<\beta^{\prime}<\alpha_{N}^{\prime}$ is satisfied if and only if $A A_{0}=\frac{d_{0}^{2}}{2 c_{A_{0}}|w|^{2} N^{2}}+$ $O\left(N^{-3}\right)$. Therefore, using the fact that $\alpha_{N}^{\prime}-\alpha_{N}=O\left(N^{-2}\right)$, we obtain

$$
I_{N}^{+}=\int_{0}^{\frac{d_{0}^{2}}{2 \mathfrak{c}_{A_{0}}|w|^{2} N^{2}}}+O\left(N^{-3}\right)\left(\alpha_{N}^{\prime}-\alpha_{N}\right) d x=\frac{d_{0}^{3} B^{\prime \prime} B_{0}}{2 \mathfrak{c}_{A_{0}}|w|^{4} N^{4}}+O\left(N^{-5}\right),
$$

where we used (28). Now (27) combined with (22) implies that $\beta<\alpha_{N}^{\prime}$ if and only if

$$
\frac{A A_{0}}{A_{0} A_{0}^{\prime}}+\frac{\left(A A_{0}\right)^{2}}{2\left(A_{0} A_{0}^{\prime}\right)^{3} \mathfrak{c}_{A_{0}^{\prime}}}+O\left(\left(A A_{0}\right)^{3}\right)<\frac{d_{0}+A_{0} A}{\frac{w \cdot \overline{A_{0} B_{N}^{\prime \prime}}}{|w|}}+O\left(N^{-3}\right)
$$

i.e. $A_{0} A<\mathfrak{T}_{N}^{\prime}:=\frac{d_{0} A_{0} A_{0}^{\prime}}{\frac{w \cdot \overline{A_{0} B_{N}^{\prime \prime}}}{|w|}}+\left(d_{0}\left(A_{0} A_{0}^{\prime}\right)^{2}-\frac{d_{0}^{2}}{2 \mathfrak{c}_{A_{0}^{\prime}}}\right) \frac{1}{\left(\frac{w \cdot A_{0} B_{N}^{\prime \prime}}{|w|}\right)^{2}}+O\left(N^{-3}\right)$. Analogously $\beta<\alpha_{N}$ if and only if

$$
A_{0} A<\mathfrak{T}_{N}:=\frac{d_{0} A_{0} A_{0}^{\prime}}{\frac{w \cdot \overrightarrow{A_{0} B_{N}}}{|w|}}+\left(d_{0}\left(A_{0} A_{0}^{\prime}\right)^{2}-\frac{d_{0}^{2}}{2 \mathfrak{c}_{A_{0}^{\prime}}}\right) \frac{1}{\left(\frac{w \cdot \overrightarrow{A_{0} B_{N}}}{|w|}\right)^{2}}+O\left(N^{-3}\right) .
$$

So $\mathfrak{T}_{N}^{\prime}-\mathfrak{T}_{N} \sim \frac{d_{0} A_{0} A_{0}^{\prime} B^{\prime \prime} B_{0}}{\left(\frac{w \cdot \overrightarrow{A_{0} B_{N}}}{|w|}\right)^{2}}$. Since $\alpha_{N}=O\left(N^{-1}\right)$ and $\alpha_{N}^{\prime}-\alpha_{N}=O\left(N^{-2}\right)$, it follows that

$$
I_{N}^{-}=\int_{-\mathfrak{T}_{N}^{\prime}}^{0}\left(\alpha_{N}^{\prime}-\max \left(\beta, \alpha_{N}\right)\right) d x+O\left(N^{-5}\right) .
$$

Now observe that $\alpha_{N}<\beta<\alpha_{N}^{\prime}$ if and only if $\mathfrak{T}_{N}<A_{0} A<\mathfrak{T}_{N}^{\prime}$ and in this case:

$$
\alpha_{N}^{\prime}-\max \left(\beta, \alpha_{N}\right)=\alpha_{N}^{\prime}-\beta=\left(\mathfrak{T}_{N}^{\prime}-A A_{0}\right)\left(\frac{1}{A_{0} A_{0}^{\prime}}-\frac{1}{\frac{w \cdot \overrightarrow{A_{0} B_{N}^{\prime \prime}}}{|w|}}\right)+O\left(N^{-3}\right) .
$$


Otherwise $\alpha_{N}^{\prime}-\max \left(\beta, \alpha_{N}\right)=\alpha_{N}^{\prime}-\alpha_{N}$ and so

$$
\alpha_{N}^{\prime}-\max \left(\beta, \alpha_{N}\right)=\left(d_{0}+A_{0} A\right)\left(\frac{1}{\frac{w \cdot \overrightarrow{A_{0} B_{N}^{\prime \prime}}}{|w|}}-\frac{1}{\frac{w \cdot \overrightarrow{A_{0} B_{N}}}{|w|}}\right)+\frac{d_{0}^{2}}{2\left(\frac{w \cdot \overrightarrow{A_{0} B_{N}}}{|w|}\right)^{3}}\left(\frac{1}{\mathfrak{c}_{B_{0}}}-\frac{1}{\mathfrak{c}_{B^{\prime \prime}}}\right)+o\left(N^{-3}\right) .
$$

Therefore $I_{N}^{-}=\frac{\Gamma_{1}}{\left(\frac{w \cdot \overrightarrow{A_{0} B_{N}}}{|w|}\right)^{3}}+\frac{\Gamma_{2}}{\left(\frac{w \cdot \overrightarrow{A_{0} B_{N}}}{|w|}\right)^{4}}+o\left(N^{-4}\right)$, with $\Gamma_{1}:=d_{0}^{2} A_{0} A_{0}^{\prime} B^{\prime \prime} B_{0}$ and

$$
\Gamma_{2}:=\frac{3 d_{0}^{2} A_{0} A_{0}^{\prime} B^{\prime \prime} B_{0}\left(A_{0} A_{0}^{\prime}+B^{\prime \prime} B_{0}\right)}{2}-\frac{d_{0}^{3} B^{\prime \prime} B_{0}}{2 \mathfrak{c}_{A_{0}^{\prime}}}+\frac{d_{0}^{3} A_{0} A_{0}^{\prime}}{2}\left(\frac{1}{\mathfrak{c}_{B_{0}}}-\frac{1}{\mathfrak{c}_{B^{\prime \prime}}}\right) .
$$

Thus, due to (20), (21) and (29), we conclude that

$$
\bar{\mu}\left(M_{\left(i_{0}, 0\right)} \cap T^{-1}\left(M_{\left(i_{1}, L+N w\right)}\right)\right)=\frac{\Gamma_{1}}{2|\partial \bar{Q}|\left(\frac{w \cdot \overline{A_{0} B_{N}}}{|w|}\right)^{3}}+\frac{\Gamma_{3}}{2|\partial \bar{Q}|\left(\frac{w \cdot \overline{A_{0} B_{N}}}{|w|}\right)^{4}}+o\left(N^{-4}\right),
$$

with $\Gamma_{3}:=\Gamma_{2}+\frac{d_{0}^{3} B^{\prime \prime} B_{0}}{2 \mathfrak{c}_{A_{0}}}$. We obtain (19) by noticing that, since $\overrightarrow{B B_{N}}=N w$,

$$
\frac{1}{\left(\frac{w \cdot \overrightarrow{A_{0} B_{N}}}{|w|}\right)^{3}}=\frac{1}{\left(N|w|+\frac{w \cdot \overrightarrow{A_{0} B_{0}}}{|w|}\right)^{3}}=\frac{1}{(N|w|)^{3}}\left(1-3 \frac{w \cdot \overrightarrow{A_{0} B_{0}}}{N|w|^{2}}\right)+o\left(N^{-4}\right),
$$

and analogously that $\frac{1}{\left(\frac{w \cdot \overline{A_{0} B_{N}}}{|w|}\right)^{4}}=\frac{1}{(N|w|)^{4}}+o\left(N^{-4}\right)$.

\section{Regularity of the Projector $t \mapsto \Pi_{t}$ at $t=0$}

In this section we state and prove Proposition 5.3, which is a generalization of Proposition 3.1. We do not assume (12). We write $\pi_{0}: \Delta \rightarrow Y$ for the vertical projection from $\Delta$ to its base $Y=\Delta_{0}$ given by $\pi_{0}(x, \ell)=(x, 0)$ and $\omega: \Delta \rightarrow \mathbb{N}$ for the level map given by $\omega((x, \ell))=\ell$. To simplify notations we write $\mu_{\Delta}(h)$ for $\int_{\Delta} h d \mu_{\Delta}$.

5.1. Banach spaces and regularity of the dominating eigenprojector. We start by recalling results from $[6,7,30,32]$. First recall that the operator $P$ can be written as follows

$$
\forall h \in L^{1}\left(\mu_{\Delta}\right), \quad P h(x)=\sum_{Y \in f^{-1}(x)} e^{-\alpha(y)} h(y), \quad x \in \Delta,
$$

with $\alpha \equiv 0$ outside the base of $\Delta$. We write $s_{0}(\cdot, \cdot)$ for the separation time for $f$ on $\Delta$ corresponding to the separation time $s(\cdot, \cdot)$ in [32]. In particular, if $s_{0}(x, y) \geq n$ then the corresponding elements in $\bar{M}$ (or in $M_{N}$ ) are in the same atom of $\xi_{0}^{n}$. Recall the class of $\eta \in(0,1)$-Hölder functions defined in (13). Choose $\beta \in(\eta, 1)$ large enough so that $\left|\alpha\left(y_{1}\right)-\alpha\left(y_{2}\right)\right| \leq C_{\alpha} \beta^{s_{0}\left(y_{1}, y_{2}\right)+1}$ if $s_{0}\left(y_{1}, y_{2}\right) \geq 1$. The condition $\beta>\eta$ will ensure that the Banach spaces $\mathcal{B}_{0}$ and $\mathcal{B}$ described below will be tailored to the approximation of observables considered in Theorem 2.4. We define the space $\mathcal{B}_{0}$ of Lipschitz functions $h: \Delta \rightarrow \mathbb{C}$ with respect to the metric $\beta^{s_{0}(\cdot, \cdot)}$, with norm

$$
\|h\|_{\mathcal{B}_{0}}:=\|h\|_{\infty}+\operatorname{ess} \sup _{x, y \in \Delta: s_{0}(x, y) \geq 1} \frac{|h(x)-h(y)|}{\beta^{s_{0}(x, y)}} .
$$


Let $p>2$ and choose $\varepsilon>0$ small enough so that in particular $\sum_{\ell>0} e^{p \ell \varepsilon} \mu_{\Delta}\left(\Delta_{\ell}\right)<\infty$, writing $\Delta_{\ell}$ for the $\ell$-th floor of the tower $\Delta$. We let $\mathcal{B}$ be the space of functions $h: \Delta \rightarrow \mathbb{C}$ such that the following quantity is finite

$$
\|h\|_{\mathcal{B}}:=\sup _{\ell \geq 1} e^{-\ell \varepsilon}\left(\left\|h_{\mid \Delta_{\ell}}\right\|_{\infty}+\operatorname{ess} \sup _{x, y \in \Delta_{\ell}: s_{0}(x, y) \geq 1} \frac{|h(x)-h(y)|}{\beta^{s_{0}(x, y)}}\right) .
$$

The choice of $\varepsilon$ ensures that the Young Banach space $\mathcal{B}$ can be continuously injected in $L^{p}\left(\mu_{\Delta}\right)$. While $\mathcal{B}_{0}$ is independent of $p, \mathcal{B}$ depends on $p$ via $\varepsilon$.

Lemma 5.1. Let $H: g \mapsto P(\hat{\kappa} g)$. Then $H^{3}(\mathbf{1}) \notin L^{1}\left(\mu_{\Delta}\right)$. As a consequence, since $\mathbf{1} \in \mathcal{B} \subset L^{1}\left(\mu_{\Delta}\right), P_{0}^{\prime}=i H$ does acts neither on $\mathcal{B}$ nor on $L^{1}\left(\mu_{\Delta}\right)$

Proof. Note that $H^{3}(\mathbf{1})=P(\hat{\kappa} P(\hat{\kappa} P(\hat{\kappa})))=P^{3}\left(\hat{\kappa} \circ f^{2} . \hat{\kappa} \circ f . \hat{\kappa}\right)$, using the fact that $\hat{\kappa} P(g)=P(g . \hat{\kappa} \circ f)$. In particular $H^{3}(\mathbf{1})$ is integrable if and only if $\kappa \circ \bar{T}^{2} . \kappa \circ \bar{T} . \kappa$ is integrable, i.e. if and only if $\kappa \circ \bar{T} . \kappa . \kappa \circ \bar{T}^{-1}$. Let us prove that this random variable is not integrable. Due to [30, Proposition 9], there exist $K, K_{0}>0$ such that $\left|\kappa \circ \bar{T}^{-1}\right|$ and $|\kappa \circ \bar{T}|$ are both larger that $K \sqrt{|\kappa|}-K_{0}$. Since $\kappa^{2}$ is not integrable, nor is $\left|\kappa \circ \bar{T} . \kappa . \kappa \circ \bar{T}^{-1}\right|$.

In what follows we exploit that $\mathcal{B}$ is continuously embedded in $L^{p}\left(\mu_{\Delta}\right)$ and satisfies (15) and (16). Using just the information on big tail $\bar{\mu}(|\kappa|>N)=O\left(N^{-2}\right)$,

Lemma 5.2. Let $q \in\left[1, \frac{2 p}{p+2}\right)$ (so that $\left.2\left(\frac{1}{q}-\frac{1}{p}\right)>1\right)$ and $\gamma \in\left(1,2\left(\frac{1}{q}-\frac{1}{p}\right)\right.$ ), there exists $C=C(q, \gamma)>0$ such that for all $t \in[-\pi, \pi]^{2},\left\|P_{t}-P_{0}-t \cdot P_{0}^{\prime}\right\|_{\mathcal{B} \rightarrow L^{q}} \leq C t^{\gamma}$, with $P_{0}^{\prime}:=P(i \kappa \cdot)$.

Proof. Note that $q<p$. Set $r:=\frac{q p}{p-q}$ so that $\frac{1}{q}=\frac{1}{p}+\frac{1}{r}$. With this notation, the upper bound on $\gamma$ can be rewritten $\gamma r<2$ and

$$
\begin{aligned}
\| P_{t}- & P_{0}-t \cdot P_{0}^{\prime}\left\|_{\mathcal{B} \rightarrow L^{q}} \ll\right\| P_{t}-P_{0}-t \cdot P_{0}^{\prime} \|_{L^{p} \rightarrow L^{q}} \\
& \leq\left\|v \mapsto P\left(\left(e^{i t \cdot \hat{\kappa}}-1-i t \cdot \hat{\kappa}\right) v\right)\right\|_{L^{p} \rightarrow L^{q}} \\
& \leq\left\|v \mapsto\left(e^{i t \cdot \hat{\kappa}}-1-i t \cdot \hat{\kappa}\right) v\right\|_{L^{p} \rightarrow L^{q}} \leq\left\|e^{i t \cdot \hat{\kappa}}-1-i t \cdot \hat{\kappa}\right\|_{L^{r}} \\
& \leq\left\|t^{\gamma} \hat{\kappa}^{\gamma}\right\|_{L^{r}}=t^{\gamma}\left(\mathbb{E}\left[\hat{\kappa}^{\gamma r}\right]\right)^{1 / r}=O\left(t^{\gamma}\right),
\end{aligned}
$$

where we used the Hölder inequality at the penultimate line and the fact that $\hat{\kappa}$ admits moments of every order smaller than 2 at the last line. We have also used the fact that $\left|e^{i \hat{\kappa}}-1-i \hat{\kappa}\right| \leq \min \left(|\hat{\kappa}|,|\hat{\kappa}|^{2}\right) \leq|\hat{\kappa}|^{\gamma}$ for any $\gamma \in[1,2]$ and for all $x$ and that $\gamma r<2$.

Given $q \in[1,2)$ up to taking $p>2 q /(2-q)$ large enough (and so up to our choice of $\mathcal{B}$ ), we can adjust $\gamma$ so that $\gamma<2 / q$ is as close to $2 / q$ as we wish (in particular as close to 2 as we wish if $q=1)$. Let $Y:=\Delta_{0}$ be the base of the tower $\Delta$. Throughout, we let $\mu_{Y}=\mu_{\Delta}(\cdot \mid Y)$. As shown in [7,30], the height of the tower $\left(\Delta, f, \mu_{\Delta}\right)$, which we denote by $\sigma: Y \rightarrow \mathbb{N}$, has exponential tail $\mu_{Y}(\sigma>n) \ll \theta_{1}^{n}$ for some $\theta_{1} \in(0,1)$. Using Lemma 5.2 and building on the arguments used in [2], we obtain the following expansion of eigenprojector $\Pi_{t}$. 
Proposition 5.3. Let $b \in(p,+\infty]$. For every $w \in \mathcal{B}_{0}$, every $v \in L^{b}\left(\mu_{\Delta}\right)$ constant on each $(a, \ell)$ (with $a \in \alpha$ and $0 \leq \ell<\sigma(a)$ ) such that $v w \in \mathcal{B}$, there exists $\Pi_{0}^{\prime}(v w)$ belonging to $L^{\eta}\left(\mu_{\Delta}\right)$ for every $\eta \in[1,2)$ such that $1_{Y} \Pi_{0}^{\prime}(v w) \in \mathcal{B}_{0}$.

Moreover, for every $p^{\prime} \in(1,4 / 3)$ and every $\gamma \in\left(1, \min \left(2 \frac{p-1}{p}, \frac{4}{p^{\prime}}-2\right)\right)$, there exists $C, \delta>0$ such that, for every $(v, w)$ as above and all $t \in B_{\delta}(0)$,

$$
\left\|\left(\Pi_{t}-\Pi_{0}-t \cdot \Pi_{0}^{\prime}\right)(v w)\right\|_{L^{p^{\prime}\left(\mu_{\Delta}\right)}} \leq C|t|^{\gamma}\left(\|v w\|_{\mathcal{B}}+\|w\|_{\mathcal{B}_{0}}\|v\|_{L^{b}\left(\mu_{\Delta}\right)}\right) .
$$

and $\left\|\Pi_{0}^{\prime}(v w)\right\|_{L^{\eta}} \leq C\left(\|v w\|_{\mathcal{B}}+\|w\|_{\mathcal{B}_{0}}\|v\|_{L^{b}\left(\mu_{\Delta}\right)}\right)$. Moreover $\Pi_{0}^{\prime}$ satisfies

$$
\Pi_{0}^{\prime}(v w)(x)=\Pi_{0}^{\prime}(v w) \circ \pi_{0}(x)+i \sum_{m=0}^{\omega(x)-1} \hat{\kappa} \circ f^{m}\left(\pi_{0}(x)\right) \int_{\Delta} v w d \mu_{\Delta} .
$$

We postpone the proof of Proposition 5.3 to the end of this section. We remark that Proposition 5.3 cannot be proved merely via the continuity arguments in [16], which is why we resort to building on the arguments in [2]. The key elements used in the proof of Proposition 5.3 are : (i) obtain Lemma 5.12, which gives the expansion of $t \mapsto \int_{Y} \Pi_{t} v d \mu_{Y}$ for suitable functions $v$, as in Proposition 5.4; (ii) the key observation in (i) is that it is sensible to study the smoothness in $t$ of $\int_{Y} \Pi_{t} d \mu_{Y}$, first and use this to define $\mathbf{1}_{Y} \Pi_{0}^{\prime}$ and further, to control $\left\|1_{Y} \Pi_{0}^{\prime} v\right\|_{\mathcal{B}_{0}}$ for suitable functions $v$. (iii) use (i) and (ii) together with formula (33) to control $\Pi_{0}^{\prime} v$ in $L^{p^{\prime}}$ for suitable $v$ and $p^{\prime}$. For further details on the use of Lemma 5.12 in defining $\mathbf{1}_{Y} \Pi_{0}^{\prime}$ we refer to the last paragraph in Sect. 5.2.

5.2. Regularity of $t \mapsto \mathbf{1}_{Y} \Pi_{t}$. Recall that $\sigma$ corresponds to the first return time of $f$ to the base $Y$. In what follows, we let $F=f^{\sigma}: Y \rightarrow Y$ with $F(y)=f^{\sigma(y)}(y)$ for all $y \in Y$ be the first return map. Recall that $\left(Y, F, \mu_{Y}\right)$ is a Gibbs Markov map with respect to a suitable countable partition $\mathcal{Y}$ and that $\sigma$ is constant on each atom of $\mathcal{Y}$ (the required definitions are recalled below). Let $R: L^{1}\left(\mu_{Y}\right) \rightarrow L^{1}\left(\mu_{Y}\right)$ be the transfer operator of the Gibbs Markov base map $\left(Y, F=f^{\sigma}, \mu_{Y}\right)$ given by

$$
R v(x):=\sum_{y: F y=x} \chi(y) v(y)=\sum_{n \geq 1} P^{n}\left(1_{Y \cap\{\sigma=n\}} v\right),
$$

with $\chi=\frac{d \mu_{Y}}{d \mu_{Y} \circ F}: Y \rightarrow \mathbb{R}$. In particular, on $f^{-1}(Y), e^{-\alpha}=\chi \circ \pi_{0}$. Define $d_{\beta}\left(y, y^{\prime}\right)=$ $\beta^{s\left(y, y^{\prime}\right)}$ where the separation time for $F, s\left(y, y^{\prime}\right)$, is the least integer $n \geq 0$ such that $F^{n} y$ and $F^{n} y^{\prime}$ lie in distinct partition elements in $\alpha$. The partition $\mathcal{Y}$ separates trajectories with $s\left(y, y^{\prime}\right)=\infty$ if and only if $y=y^{\prime}$; so $d_{\beta}$ is a metric. The map $F$ is a (full-branch) Gibbs-Markov map, which means that

- $\left.F\right|_{a}: a \rightarrow Y$ is a measurable bijection for each $a \in \mathcal{Y}$, and

- $\left|\log \chi(y)-\log \chi\left(y^{\prime}\right)\right| \leq C_{\alpha} d_{\beta}\left(y, y^{\prime}\right)$ for all $y, y^{\prime} \in a, a \in \mathcal{Y}($ since $s(\cdot, \cdot) \leq$ $\left.s_{0}(\cdot, \cdot)\right)$.

A consequence of this definition is that there is a constant $C>0$ such that

$$
\chi(y) \leq C \mu_{Y}(a) \quad \text { and } \quad\left|\chi(y)-\chi\left(y^{\prime}\right)\right| \leq C \mu_{Y}(a) d_{\beta}\left(y, y^{\prime}\right),
$$

for all $a \in \mathcal{Y}$ and $y, y^{\prime} \in a$. Since $F$ is Gibbs Markov, it follows that (see, for instance, [26, Section 5]): 
- The space $\left(\mathcal{B}_{1},\|\cdot\|_{\mathcal{B}_{0}}\right)$ of $\beta$-Hölder continuous functions on $Y$ contains constant functions and $\mathcal{B}_{1} \subset L^{\infty}\left(\mu_{Y}\right)$. Note that $\mathcal{B}_{1}$ corresponds to functions $h \in \mathcal{B}_{0}$ supported on $Y$. For this reason and for the reader convenience, we have chosen to write also $\|\cdot\|_{\mathcal{B}_{0}}$ for the norm of $\mathcal{B}_{1}$ (in order to avoid the introduction of unnecessary notation).

- $R$ is quasi-compact on $\mathcal{B}_{1}$ and 1 is a simple eigenvalue for $R$, isolated in the spectrum of $R$.

Building on the argument of [2, Lemma 3.15], in this section we obtain

Proposition 5.4. Let $b \in(p,+\infty]$ and $\gamma \in\left(1,2 \frac{p-1}{p}\right)$. There exists $C>0$ such that, for every $w \in \mathcal{B}_{0}$ and $v \in L^{b}\left(\mu_{\Delta}\right)$ constant on each $(a, \ell)$ (with $a \in \alpha$ and $0 \leq \ell<\sigma(a)$ ) so that $v w \in \mathcal{B}$, the following holds true

$$
\begin{aligned}
& \left\|1_{Y}\left(\Pi_{t}-\Pi_{0}-t \cdot \Pi_{0}^{\prime}\right)(v w)\right\|_{\mathcal{B}_{0}} \leq C|t|^{\gamma}\left(\|v w\|_{\mathcal{B}}+\|w\|_{\mathcal{B}_{0}}\|v\|_{L^{b}\left(\mu_{\Delta}\right)}\right), \\
& \text { with } \quad 1_{Y} \Pi_{0}^{\prime}(v w):=\mu_{\Delta}(v w) Q_{0}^{\prime}\left(1_{Y}\right)+c_{0}(v w) 1_{Y} \in \mathcal{B}_{0}, \\
& \text { and } \quad c_{0}(v w):=i\left(\sum_{j \geq 0} \int_{\Delta} \hat{\kappa} \circ f^{j} . v w d \mu_{\Delta}+\mu_{\Delta}(v w) \sum_{j \geq 0} \int_{\Delta} \hat{\kappa} \frac{1_{Y} \circ f^{j+1}}{\mu_{\Delta}(Y)} d \mu_{\Delta}\right) \text {, }
\end{aligned}
$$

and with $Q_{0}^{\prime}\left(1_{Y}\right) \in \mathcal{B}_{1}$ given by (46) and $\left\|1_{Y} \Pi_{0}^{\prime}(v w)\right\|_{\infty} \leq C^{\prime}\|w\|_{\mathcal{B}_{0}}\|v\|_{L^{b}\left(\mu_{\Delta}\right)}$.

Remark 5.5. The expression of $c_{0}(v w)$ comes from Lemma 5.12. As shown in Lemma C. 2 below, the sums in the formula for $c_{0}(v w)$ are absolutely convergent and in $O\left(\|w\|_{\mathcal{B}_{0}}\|v\|_{L^{b}\left(\mu_{\Delta}\right)}\right)$.

Remark 5.6. Under the assumptions of Proposition 5.3, if $\mu_{\Delta}(v w)=0$, then

$$
\Pi_{0}^{\prime}(v w)=\Pi_{0}^{\prime}(v w) \circ \pi_{0}=c_{0}(v w)=i \sum_{j \geq 0} \int_{\Delta} \hat{\kappa} \circ f^{j} . v w d \mu_{\Delta} .
$$

If moreover $v w=v^{\prime} w^{\prime}-\left(v^{\prime} w^{\prime}\right) \circ f$ is a coboundary of functions of the same kind, then

$$
\Pi_{0}^{\prime}(v w)=\Pi_{0}^{\prime}(v w) \circ \pi_{0}=c_{0}(v w)=-i \int_{\Delta} \hat{\kappa} \cdot\left(v^{\prime} w^{\prime}\right) \circ f d \mu_{\Delta} .
$$

Recall that $\sigma$ is the first return time of $f$ to the base $Y$. Write $\hat{\kappa}_{n}:=\sum_{j=0}^{n-1} \hat{\kappa} \circ f^{j}$ and note that $\hat{\kappa}_{\sigma}:=\sum_{j=0}^{\sigma(\cdot)-1} \hat{\kappa} \circ f^{j}$ is the induced (to the base $Y$ ) version of $\hat{\kappa}$. In order to define $1_{Y} \Pi_{0}^{\prime} v$ we will justify that the derivative at $t=0$ of LHS of the following identity

$$
1_{Y} \Pi_{t}(v w)=\int_{Y} \Pi_{t}(v w) d \mu_{Y} \frac{Q_{t}\left(1_{Y}\right)}{\int_{Y} Q_{t}\left(1_{Y} v w\right) d \mu_{Y}}
$$

is well defined. Here, $Q_{t}$ is an eigenprojector of a perturbation $\tilde{R}_{t}$ of $R$. More precisely, while $\Pi_{t}$ is the eigenprojector of $P\left(\lambda_{t}^{-1} e^{i t \hat{\kappa}} \cdot\right)$ associated to the eigenvalue $1, Q_{t}$ is the main eigenprojector of $\tilde{R}_{t}=R\left(\lambda_{t}^{-\sigma(\cdot)} e^{i t \hat{\kappa}_{\sigma}} \cdot\right.$ ) associated to the eigenvalue 1 (see below for the formal definition of $\tilde{R}_{t}$ ). The above displayed formula allows us to exploit that in the RHS we only have $\int_{Y} \Pi_{t}(v w) d \mu_{Y}$ and $Q_{t}$. As explained below $Q_{t}$ is much easier to understand than $\Pi_{t}$. Among other technical lemmas, in the next subsection, we obtain the required expansion (in norm) for $Q_{t}$ (see Lemma 5.11). Lemma 5.12 below allows us to control the derivative of $\int_{Y} \Pi_{t}(v w) d \mu_{Y}$ at 0 . 
5.3. Technical lemmas. We start with the following lemma on the integrability of $\hat{\kappa}_{\sigma}$.

Lemma 5.7. For any $r \in(1,2), \int_{Y}\left|\hat{\kappa}_{\sigma}\right|^{r} d \mu_{Y}<\infty$.

Proof. First, due to the Hölder inequality,

$$
\int_{Y}\left|\hat{\kappa}_{\sigma}\right|^{r} d \mu_{Y}=\sum_{n \geq 1} \int_{Y \cap\{\sigma=n\}}\left|\hat{\kappa}_{n}\right|^{r} d \mu_{Y} \leq \sum_{n \geq 1} \mu(\sigma=n)^{1 / q} \int_{Y}\left|\hat{\kappa}_{n}\right|^{r p} 1_{\{\sigma=n\}} d \mu_{Y},
$$

with $p \in(1,2 / r)$ and $q=p /(p-1)$ so that $1 / p+1 / q=1$. Let $s=r p /(r p-1)$ so that $1 /(r p)+1 / s=1$. Using the Hölder inequality for inner products, we have that for any $n \geq 1$

$$
\begin{aligned}
\left|\hat{\kappa}_{n}\right|^{r p} & =\left|\sum_{j=0}^{n-1} \hat{\kappa} \circ f^{j} \cdot 1\right|^{r p} \leq\left(\left|\sum_{j=0}^{n-1} 1\right|^{1 / s}\left(\sum_{j=0}^{n-1}\left|\hat{\kappa} \circ f^{j}\right|^{r p}\right)^{1 / r p}\right)^{r p} \\
& \leq n^{r p / s} \sum_{j=0}^{n-1}\left|\hat{\kappa} \circ f^{j}\right|^{r p},
\end{aligned}
$$

and thus $\int_{Y}\left|\hat{\kappa}_{\sigma}\right|^{r} d \mu_{Y} \leq \sum_{n \geq 1} \mu(\sigma=n)^{1 / q} n^{r p / s} \int_{Y} \sum_{j=0}^{n-1}\left|\hat{\kappa} \circ f^{j}\right|^{r p} 1_{\{\sigma=n\}} d \mu_{Y}$ which leads to

$$
\int_{Y}\left|\hat{\kappa}_{\sigma}\right|^{r} d \mu_{Y} \leq \sum_{n \geq 1} C_{1} \theta_{1}^{n / q} n^{r p / s}(\mu(Y))^{-1} \int_{\Delta}|\hat{\kappa}|^{r p} d \mu_{\Delta}<\infty .
$$

We define $\tilde{R}_{t}:=\sum_{n \geq 1} \lambda_{t}^{-n} P_{t}^{n}\left(1_{Y \cap\{\sigma=n\}} v\right)=\sum_{n>1} \lambda_{t}^{-n} R_{n}\left(e^{i t \hat{\kappa}_{n}} \cdot\right)$, with $R_{n} v:=$ $R\left(1_{\{\sigma=n\}} v\right)=P^{n}\left(1_{Y \cap\{\sigma=n\}} v\right)$. The next lemma provides some useful estimates on $R_{n}$.

Lemma 5.8. Let $b \in(2,+\infty]$. Fix $q \in\left(\frac{b}{b-1}, 2\right)$ (with convention $\frac{b}{b-1}=1$ if $b=\infty$ ) and $\varepsilon_{0} \in(0,1)$. Then, for every $\gamma \in[1,2 / q)$, there exist $C_{0}$ and $\rho \in(0,1)$ so that for all $t$ small enough, all $w \in \mathcal{B}_{1}$, all $n \geq 1$ and all $v_{Y} \in L^{b}\left(\mu_{Y}\right)$ constant on each atom of the partition $\alpha$,

$$
\begin{aligned}
\left\|R_{n}\left(\left(e^{i t \hat{\kappa}_{\sigma}}-1-i t \hat{\kappa}_{\sigma}\right) v_{Y} w\right)\right\|_{\mathcal{B}_{0}} & \leq C_{0} \rho^{n}|t|^{\gamma}\left\|\hat{\kappa}_{\sigma}\right\|_{L^{\gamma q}\left(\mu_{Y}\right)}^{\gamma}\left\|v_{Y}\right\|_{L^{b}\left(\mu_{Y}\right)}\|w\|_{\mathcal{B}_{0}} \\
\left\|R_{n}\left(\hat{\kappa}_{\sigma} v_{Y} w\right)\right\|_{\mathcal{B}_{0}} & \leq C_{0} \rho^{n}\left\|v_{Y}\right\|_{L^{b}\left(\mu_{Y}\right)}\left\|\hat{\kappa}_{\sigma}\right\|_{L^{\gamma q}\left(\mu_{Y}\right)}^{\gamma}\|w\|_{\mathcal{B}_{0}}, \\
\left\|R_{n}\left(v_{Y} w\right)\right\|_{\mathcal{B}_{0}} & \leq C_{0} \rho^{n}\left\|v_{Y}\right\|_{L^{2}\left(\mu_{Y}\right)}\|w\|_{\mathcal{B}_{0}} .
\end{aligned}
$$

Note that, in this lemma, $1<\gamma<2\left(1-\frac{1}{b}\right)$ and that up to adapting the value of $q$, we can take $\gamma$ as close to $2\left(1-\frac{1}{b}\right)$ as we wish.

Proof. By the arguments used in [20, Proof of Proposition 12.1] and exploiting that $v_{Y}$ and $\hat{\kappa}_{\sigma}$ are constant on every $a \in \mathcal{A}$,

$$
\forall w \in \mathcal{B}_{1}, \forall h \in L^{1}\left(\mu_{Y}\right), \quad\left\|R_{n}(h w)\right\|_{\mathcal{B}_{0}} \ll\left\|1_{\{\sigma=n\}} h\right\|_{L^{1}\left(\mu_{Y}\right)}\|w\|_{\mathcal{B}_{0}},
$$

for $h \in\left\{\left(e^{i t \hat{\kappa}_{\sigma}}-1-i t \hat{\kappa}_{\sigma}\right) v_{Y}, v_{Y}, \hat{\kappa}_{\sigma}, \hat{\kappa}_{\sigma} v_{Y}\right\}$. A justification of (40) based on [20, Proof of Proposition 12.1] is provided in Appendix Appendix B. We note that since 
$\sigma$ has exponential tail, equation (40) and Hölder inequality imply (38) and (39). Next, by Lemma 5.7, for any $r \in(1,2), \hat{\kappa}_{\sigma} \in L^{r}\left(\mu_{Y}\right)$. Since $\gamma q \in[1,2)$, using the same argument as in the proof of Lemma 5.2 combined with Hölder inequality, we have

$$
\int_{Y} 1_{\{\sigma=n\}}\left|\left(e^{i t \cdot \hat{\kappa}_{\sigma}}-1-i t \cdot \hat{\kappa}_{\sigma}\right) v_{Y}\right| d \mu_{Y} \leq\left\|v_{Y}\right\|_{L^{b}\left(\mu_{Y}\right)}\left\|\left(t \cdot \hat{\kappa}_{\sigma}\right)^{\gamma^{\gamma}}\right\|_{L^{q}\left(\mu_{Y}\right)}\left(\mu_{Y}(\sigma=n)\right)^{1-\frac{1}{b}-\frac{1}{q}},
$$

which leads to (37). Note that $\gamma q<2$ ensures that $\hat{\kappa}_{\sigma}^{\gamma} \in L^{q}\left(\mu_{Y}\right)$. The result follows from the previous two displayed inequalities since $\sigma$ has exponential tail.

Note that $\tilde{R}_{0}=R$ and that (18) implies that $\lambda_{0}^{\prime}=\left.\frac{d}{d t} \lambda_{t}\right|_{t=0}=0$. The next lemma shows that $t \mapsto \tilde{R}_{t} \in \mathcal{B}_{1}$ is differentiable at $t=0$ with derivative $\tilde{R}_{0}^{\prime}:=R\left(i \hat{\kappa}_{\sigma} \cdot\right)=$ $\sum_{n=0}^{\infty} R_{n}\left(i \hat{\kappa}_{n} \cdot\right)$, and that this is also true if we replace $w \in \mathcal{B}_{1}$ by $v_{Y} w$ as in Lemma 5.8.

Lemma 5.9. Let $b, q, \gamma$ as in Lemma 5.8. Then for every $\gamma \in[1,2 / q)$, there exists $C>0$ such that for $t$ small enough and all $w \in \mathcal{B}_{1}$ and for any $v_{Y}$ as in Lemma 5.8,

$$
\begin{aligned}
&\left\|R\left(v_{Y} w\right)\right\|_{\mathcal{B}_{0}} \leq C\left\|v_{Y}\right\|_{L^{b}\left(\mu_{Y}\right)}\|w\|_{\mathcal{B}_{0}}, \\
&\left\|\tilde{R}_{0}^{\prime}\left(v_{Y} w\right)\right\|_{\mathcal{B}_{0}} \leq C\left\|\hat{\kappa}_{\sigma}\right\|_{L^{\gamma q}\left(\mu_{Y}\right)}^{\gamma}\left\|v_{Y}\right\|_{L^{b}\left(\mu_{Y}\right)}\|w\|_{\mathcal{B}_{0}}, \\
&\left\|\left(\tilde{R}_{t}-\tilde{R}_{0}-t \cdot \tilde{R}_{0}^{\prime}\right)\left(v_{Y} w\right)\right\|_{\mathcal{B}_{0}} \leq C|t|^{\gamma}\left(\left\|\hat{\kappa}_{\sigma}\right\|_{L^{\gamma q}\left(\mu_{Y}\right)}^{\gamma}\left\|v_{Y}\right\|_{L^{b}\left(\mu_{Y}\right)}\right)\|w\|_{\mathcal{B}_{0}} .
\end{aligned}
$$

Proof. Summing (38) (resp. (39)) gives (42) (resp. (41)). Next, note that

$$
\begin{aligned}
& \left\|\left(\tilde{R}_{t}-\tilde{R}_{0}-t \cdot \tilde{R}_{0}^{\prime}\right)\left(v_{Y} w\right)\right\|_{\mathcal{B}_{0}} \leq \sum_{n \geq 1}\left|\lambda_{t}^{-n}\right|\left\|R_{n}\left(\left(e^{i t \cdot \hat{\kappa}_{\sigma}}-1-i t \cdot \hat{\kappa}_{\sigma}\right)\left(v_{Y} w\right)\right)\right\|_{\mathcal{B}_{0}} \\
& \quad+\sum_{n \geq 1}\left|\lambda_{t}^{-n}-1\right|\left(\left\|R_{n}\left(v_{Y} w\right)\right\|_{\mathcal{B}_{0}}+\left\|R_{n}\left(\hat{\kappa}_{\sigma}\left(v_{Y} w\right)\right)\right\|_{\mathcal{B}_{0}}\right) \\
& \leq C_{0}|t|^{\gamma}\left(\left\|\hat{\kappa}_{\sigma}\right\|_{L^{\gamma q}\left(\mu_{Y}\right)}^{\gamma}\left\|v_{Y}\right\|_{L^{b}\left(\mu_{Y}\right)}\right) \sum_{n \geq 1} \rho^{\frac{n}{2}}\|w\|_{\mathcal{B}_{0}} \\
& \quad+\left|1-\lambda_{t}\right| \sum_{n \geq 1} n\left|\lambda_{t}^{-n}\right|\left(\left\|R_{n}\left(v_{Y} w\right)\right\|_{\mathcal{B}_{0}}+t\left\|R_{n}\left(\hat{\kappa}_{\sigma}\left(v_{Y} w\right)\right)\right\|_{\mathcal{B}_{0}}\right)
\end{aligned}
$$

where in the last inequality we have used Lemma 5.8 for a suitable $\rho$ together with the fact that $\left|\lambda_{t}^{-n}-1\right|=\left|1-\lambda_{t}\right|\left|\sum_{k=1}^{n} \lambda_{t}^{-k}\right|$ and $\left|\lambda_{t}^{-1}\right|<\rho^{-\frac{1}{2}}$ if $t$ is small enough (due to (18)). We conclude by applying (38) and (39) combined with $\left|\lambda_{t}^{-1}\right|<\rho^{-\frac{1}{2}}$.

Recall that $\Pi_{t}$ acts on $\mathcal{B}$. The next lemma is a restatement of [2, Lemma 3.14] in terms of the eigenprojection $\Pi_{t}$, as opposed to the (normalized) eigenvector $\frac{\Pi_{t} 1}{\int_{\Delta} \Pi_{t} 1 d \mu_{\Delta}}$, as there.

Lemma 5.10. For any small $t$ small enough and for any $v \in \mathcal{B} \tilde{R}_{t}\left(1_{Y} \Pi_{t} v\right)=\left(1_{Y} \Pi_{t} v\right)$.

Proof. Since $\Pi_{t} v \in \mathcal{B}$, observe that $1_{Y} \Pi_{t} v \in \mathcal{B}_{1}$. For all $x \in f^{-1}(Y)$,

$$
\Pi_{t} v(x)=\lambda_{t}^{-\omega(x)} P_{t}^{\omega(x)}\left(\Pi_{t} v\right)(x)=\lambda_{t}^{-\omega(x)} e^{i t \cdot \hat{\kappa}_{\omega(x)}\left(\pi_{0}(x)\right)} \Pi_{t}(v) \circ \pi_{0}(x) .
$$

Therefore, for all $y \in Y$,

$$
\Pi_{t} v(y)=\lambda_{t}^{-1} P_{t} \Pi_{t} v(y)=\sum_{x \in f^{-1}(y)} \chi \circ \pi_{0}(x) \lambda_{t}^{-1} e^{i t \cdot \hat{\kappa}(x)} \Pi_{t}(v)(x)
$$




$$
=\sum_{z \in F^{-1}(y)} \chi(z) \lambda_{t}^{-\sigma(z)} e^{i t \cdot \hat{\kappa}_{\sigma(z)}(z)} \Pi_{t}(v)(z)=\tilde{R}_{t}\left(1_{Y} \Pi_{t} v\right)(x)
$$

due to (44), since $\omega(x)+1=\sigma\left(\pi_{0}(x)\right)$ and since, for every $x \in f^{-1}(Y), x=$ $f^{\omega(x)}\left(\pi_{0}(x)\right)$.

By Lemma 5.10, for $t$ small enough, the eigenvalue $\tilde{\lambda}_{t}$ of $\tilde{R}_{t}$ associated with the projection $\left(1_{Y} \Pi_{t}\right) v$ is so that $\tilde{\lambda}_{t}=1$; this lemma tells us how the projection acts on $\mathcal{B}$. Let $Q_{t}$ be the eigenprojection for $\tilde{R}_{t}$ associated with $\tilde{\lambda}_{t}=1$. Since 1 is an isolated eigenvalue in the spectrum of $\tilde{R}_{0}=R$ and $\tilde{R}_{t}$ is a continuous family of operators (by Lemma 5.9), we have that $\tilde{\lambda}_{t}=1$ is isolated in the spectrum of $\tilde{R}_{t}$ for every $t$ small enough. Hence, there exists $\delta_{0}>0$ so that for any $\delta \in\left(0, \delta_{0}\right)$,

$$
Q_{t}=\frac{1}{2 i \pi} \int_{|\xi-1|=\delta}\left(\xi I-\tilde{R}_{t}\right)^{-1} d \xi
$$

Since $\left(\xi I-\tilde{R}_{0}\right)^{-1}$ and $\tilde{R}_{0}^{\prime}$ are well defined operators in $\mathcal{B}_{1} \subset L^{\infty}\left(\mu_{Y}\right)$, so is the derivative at $t=0$ of $t \mapsto Q_{t} \in \mathcal{B}_{1}$ and write

$$
Q_{0}^{\prime}=\frac{1}{2 i \pi} \int_{|\xi-1|=\delta}\left(\xi I-\tilde{R}_{0}\right)^{-1} \tilde{R}_{0}^{\prime}\left(\xi I-\tilde{R}_{0}\right)^{-1} d \xi
$$

for $\delta>0$ small enough. Recall that $Q_{0}, Q_{0}^{\prime}$ are well defined in $\mathcal{B}_{1}$. The next result shows that $\left\|Q_{0}^{\prime} h\right\|_{\mathcal{B}_{0}}$ is also well defined for $h=v_{Y} w$ with $v_{Y}, w$ as in Lemma 5.8.

Lemma 5.11. Let $b, q, \gamma$ as in Lemma 5.8. Then there exist $C_{1}, C_{2}>0$ such that for $t$ small enough, for every $v_{Y} \in L^{b}\left(\mu_{Y}\right)$ constant on each $a \in \alpha$ and every $w \in \mathcal{B}_{1}$,

$$
\begin{aligned}
\left\|Q_{t}\left(v_{Y} w\right)\right\|_{\mathcal{B}_{0}} & \leq C_{1}\|w\|_{\mathcal{B}_{0}}\left\|v_{Y}\right\|_{L^{b}\left(\mu_{Y}\right)}, \\
\left\|Q_{0}^{\prime}\left(v_{Y} w\right)\right\|_{\mathcal{B}_{0}} & \leq C_{1}\left\|\hat{\kappa}_{\sigma}\right\|_{L^{\gamma q}\left(\mu_{Y}\right)}^{\gamma}\left\|v_{Y}\right\|_{L^{b}\left(\mu_{Y}\right)}\|w\|_{\mathcal{B}_{0}}, \\
\left\|\left(Q_{t}-Q_{0}-t Q_{0}^{\prime}\right)\left(v_{Y} w\right)\right\|_{\mathcal{B}_{0}} & \leq C_{2}|t|^{\gamma}\left(\left\|\hat{\kappa}_{\sigma}\right\|_{L^{\gamma q}\left(\mu_{Y}\right)}^{\gamma}\left\|v_{Y}\right\|_{L^{b}\left(\mu_{Y}\right)}\right)\|w\|_{\mathcal{B}_{0}} .
\end{aligned}
$$

Proof. The first estimate will come from our estimates of $\left\|\left(Q_{t}-Q_{0}-t Q_{0}^{\prime}\right)\left(v_{Y} w\right)\right\|_{\mathcal{B}_{0}}$ and $\left\|Q_{0}^{\prime}\left(v_{Y} w\right)\right\|_{\mathcal{B}_{0}}$ and from (45) ensuring that

$$
\begin{aligned}
\left\|Q_{0}(v w)\right\|_{\mathcal{B}_{0}}= & \frac{1}{2 \pi}\left\|\int_{|\xi-1|=\delta}\left(\xi I-\tilde{R}_{0}\right)^{-1}\left(v_{Y} w\right) d \xi\right\|_{\mathcal{B}_{0}} \\
= & \frac{1}{2 \pi} \| \int_{|\xi-1|=\delta}(\xi I-R)^{-1}\left(v_{Y} w-\mu_{Y}\left(v_{Y} w\right)\right) d \xi \\
& +\mu_{Y}\left(v_{Y} w\right) \int_{|\xi-1|=\delta}(\xi I-1)^{-1} 1_{Y} d \xi \|_{\mathcal{B}_{0}}
\end{aligned}
$$

Thus $\left\|Q_{0}(v w)\right\|_{\mathcal{B}_{0}} \leq \frac{1}{2 \pi}\left(\sum_{j \geq 1} \int_{|\xi-1|=\delta}|\xi|^{-j-1}\left\|R^{j}\left(v_{Y} w-\mu_{Y}\left(v_{Y} w\right)\right)\right\|_{\mathcal{B}_{0}} d \xi\right)+$ $\left|\mu_{Y}\left(v_{Y} w\right)\right|$. So

$$
\left\|Q_{0}(v w)\right\|_{\mathcal{B}_{0}} \ll\left(\sum_{j \geq 1}(1-\delta)^{-j-1} \rho^{j-1}\left\|v_{Y}\right\|_{L^{b}\left(\mu_{Y}\right)}\|w\|_{\mathcal{B}_{0}}\right)+\left|\mu_{Y}\left(v_{Y} w\right)\right| \ll\|w\|_{\mathcal{B}_{0}}\left\|v_{Y}\right\|_{L^{b}\left(\mu_{Y}\right)},
$$


for some $\rho \in(0,1)$, where we used (41) combined with the spectral properties of $R$, up to take $\delta$ small enough so that $(1-\delta)^{-1} \rho<1$. Second

$$
\begin{aligned}
\left(Q_{t}-Q_{0}\right)= & \frac{1}{2 i \pi} \int_{|\xi-1|=\delta}\left(\xi I-\tilde{R}_{t}\right)^{-1}\left(\tilde{R}_{0}-\tilde{R}_{t}\right)\left(\xi I-\tilde{R}_{0}\right)^{-1} d \xi \\
= & E_{1}(t)+E_{2}(t)=\frac{1}{2 i \pi} \int_{|\xi-1|=\delta}\left(\xi I-\tilde{R}_{0}\right)^{-1}\left(\tilde{R}_{0}-\tilde{R}_{t}\right)\left(\xi I-\tilde{R}_{0}\right)^{-1} d \xi \\
& +\frac{1}{2 i \pi} \int_{|\xi-1|=\delta}\left(\left(\xi I-\tilde{R}_{t}\right)^{-1}-\left(\xi I-\tilde{R}_{0}\right)^{-1}\right)\left(\tilde{R}_{0}-\tilde{R}_{t}\right)\left(\xi I-\tilde{R}_{0}\right)^{-1} d \xi
\end{aligned}
$$

Note that

$\left\|E_{2}(t)\left(v_{Y} w\right)\right\|_{\mathcal{B}_{0}} \ll \int_{|\xi-1|=\delta}\left\|\left(\xi I-\tilde{R}_{t}\right)^{-1}-\left(\xi I-\tilde{R}_{0}\right)^{-1}\right\|_{\mathcal{B}_{0}}\left\|\left(\tilde{R}_{0}-\tilde{R}_{t}\right)\left(\xi I-\tilde{R}_{0}\right)^{-1}\left(v_{Y} w\right)\right\|_{\mathcal{B}_{0}} d \xi$.

Recall that for all $\xi$ so that $|\xi-1|=\delta,\left\|\left(\xi I-\tilde{R}_{t}\right)^{-1}\right\|_{\mathcal{B}_{0}} \ll 1$, for all $t$ small enough. This together with Lemma 5.9 with $v_{Y}=1_{Y}$ implies that for all $t$ small enough,

$$
\left\|\left(\xi I-\tilde{R}_{t}\right)^{-1}-\left(\xi I-\tilde{R}_{0}\right)^{-1}\right\|_{\mathcal{B}_{0}}=\left\|\left(\xi I-\tilde{R}_{0}\right)^{-1}\left(\tilde{R}_{t}-\tilde{R}_{0}\right)\left(\xi I-\tilde{R}_{t}\right)^{-1}\right\|_{\mathcal{B}_{0}} \ll|t| .
$$

Hence $\left\|E_{2}(t)\left(v_{Y} w\right)\right\|_{\mathcal{B}_{0}} \ll|t| \int_{|\xi-1|=\delta}\left\|\left(\tilde{R}_{0}-\tilde{R}_{t}\right)\left(\xi I-\tilde{R}_{0}\right)^{-1}\left(v_{Y} w\right)\right\|_{\mathcal{B}_{0}} d \xi$. To simplify notations, we write $\mu_{Y}(\cdot)$ for $\int_{Y} \cdot d \mu_{Y}$. We claim that

$$
\int_{|\xi-1|=\delta}\left\|\left(\tilde{R}_{0}-\tilde{R}_{t}\right)\left(\xi I-\tilde{R}_{0}\right)^{-1}\left(v_{Y} w\right)\right\|_{\mathcal{B}_{0}} d \xi \ll|t|\left\|\hat{\kappa}_{\sigma}\right\|_{L^{\gamma q}\left(\mu_{Y}\right)}^{\gamma}\left\|v_{Y}\right\|_{L^{b}\left(\mu_{Y}\right)}\|w\|_{\mathcal{B}_{0}} .
$$

This implies that

$$
\left\|E_{2}(t)\left(v_{Y} w\right)\right\|_{\mathcal{B}_{0}} \ll|t|^{2}\left\|\hat{\kappa}_{\sigma}\right\|_{L^{\gamma q}\left(\mu_{Y}\right)}^{\gamma}\left\|v_{Y}\right\|_{L^{b}\left(\mu_{Y}\right)}\|w\|_{\mathcal{B}_{0}} .
$$

Next, compute that

$$
\begin{aligned}
E_{1}(t)=t Q_{0}^{\prime}+E(t)= & \frac{t}{2 i \pi} \int_{|\xi-1|=\delta}\left(\xi I-\tilde{R}_{0}\right)^{-1} \tilde{R}_{0}^{\prime}\left(\xi I-\tilde{R}_{0}\right)^{-1} d \xi \\
& +\frac{1}{2 i \pi} \int_{|\xi-1|=\delta}\left(\xi I-\tilde{R}_{0}\right)^{-1}\left(\tilde{R}_{0}-\tilde{R}_{t}-t \tilde{R}_{0}^{\prime}\right)\left(\xi I-\tilde{R}_{0}\right)^{-1} d \xi .
\end{aligned}
$$

Proceeding as in estimating $E_{2}(t)\left(v_{Y} w\right)$ above and using the first part of the conclusion in Lemma 5.9 and a formula analgous to (47),

$$
\begin{aligned}
\left\|Q_{0}^{\prime}\left(v_{Y} w\right)\right\|_{\mathcal{B}_{0}} & \ll \int_{|\xi-1|=\delta}\left\|\tilde{R}_{0}^{\prime}\left(\xi I-\tilde{R}_{0}\right)^{-1}\left(v_{Y} w\right)\right\|_{\mathcal{B}_{0}} d \xi \\
& \ll\left\|\hat{\kappa}_{\sigma}\right\|_{L^{\gamma q}\left(\mu_{Y}\right)}^{\gamma}\left\|v_{Y}\right\|_{L^{b}\left(\mu_{Y}\right)}\|w\|_{\mathcal{B}_{0}},
\end{aligned}
$$

and thus, the second part of the conclusion follows. Finally, similarly to (47), we claim that

$$
\begin{aligned}
& \left\|E(t)\left(v_{Y} w\right)\right\|_{\mathcal{B}_{0}} \ll \int_{|\xi-1|=\delta}\left\|\left(\tilde{R}_{0}-\tilde{R}_{t}-t \cdot \tilde{R}_{0}^{\prime}\right)\left(\xi I-\tilde{R}_{0}\right)^{-1}\left(v_{Y} w\right)\right\|_{\mathcal{B}_{0}} d \xi \\
& \ll|t|^{\gamma} \mid\left\|\hat{\kappa}_{\sigma}\right\|_{L^{\gamma q\left(\mu_{Y}\right)}}^{\gamma}\left\|v_{Y}\right\|_{L^{b}\left(\mu_{Y}\right)}\|w\|_{\mathcal{B}_{0}} .
\end{aligned}
$$


The third part of the conclusion follows by putting all the above together. It remains to prove the claims (47), (48) and (49). Note that for any operator $\tilde{P}$ bounded in $\mathcal{B}_{1}$, we have

$$
\begin{aligned}
& \int_{|\xi-1|=\delta}\left\|\tilde{P}\left(\xi I-\tilde{R}_{0}\right)^{-1}\left(v_{Y} w\right)\right\|_{\mathcal{B}_{0}} d \xi \\
& \quad=\int_{|\xi-1|=\delta}\left\|\tilde{P}\left(\xi I-\tilde{R}_{0}\right)^{-1}\left(v_{Y} w-\mu_{Y}\left(v_{Y} w\right) 1_{Y}\right)+(\xi-1)^{-1} \mu_{Y}\left(v_{Y} w\right) \tilde{P} 1_{Y}\right\|_{\mathcal{B}_{0}} d \xi .
\end{aligned}
$$

In the sequel, we take $\tilde{P} \in\left\{I d, \tilde{R}_{0}-\tilde{R}_{t}, \tilde{R}_{0}^{\prime}, \tilde{R}_{0}-\tilde{R}_{t}-t \tilde{R}_{0}^{\prime}\right\}$, respectively. Since $\tilde{R}_{0}=R$ has a spectral gap in $\mathcal{B}_{1}$ with decomposition $R^{j}=\mu_{Y}(\cdot) 1_{Y}+N_{j}^{\prime}$ with $\left\|N_{j}^{\prime}\right\|_{\mathcal{B}_{0}} \leq C \rho^{j}$ for some $C>0$ and some $\rho<1$, for every $j \geq 1$, we can write

$R^{j}\left(v_{Y} w-\mu_{Y}\left(v_{Y} w\right) 1_{Y}\right)=R^{j-1} R\left(v_{Y}-\mu_{Y}\left(v_{Y} w\right) 1_{Y}\right)=N_{j-1}^{\prime}\left(R\left(v_{Y} w-\mu_{Y}\left(v_{Y} w\right) 1_{Y}\right)\right)$,

since $\mu_{Y}\left(R\left(v_{Y} w-\mu_{Y}\left(v_{Y} w\right)\right)\right)=0$. We note that although $\left(v_{Y}-\mu_{Y}\left(v_{Y}\right) 1_{Y}\right) \notin \mathcal{B}_{1}$. But, by the first two displayed inequalities in Appendix Appendix B (with $v w$ replaced by $v_{Y} w$ and $\left.\mu_{Y}\left(v_{Y} w\right) 1_{Y}\right)$,

$$
\left\|R\left(v_{Y} w-\mu_{Y}\left(v_{Y} w\right)\right)\right\|_{\mathcal{B}_{0}} \leq C^{\prime}\|w\|_{\mathcal{B}_{0}}\left\|v_{Y}\right\|_{L^{1}\left(\mu_{Y}\right)},
$$

for some $C^{\prime}>0$. Thus, there exist $C^{\prime \prime}>0$ and $\rho<1$ so that for $j \geq 1$,

$$
\left\|R^{j}\left(v_{Y} w-\mu_{Y}\left(v_{Y} w\right)\right)\right\|_{\mathcal{B}_{0}} \leq C^{\prime \prime} \rho^{j}\|w\|_{\mathcal{B}_{0}}\left\|v_{Y}\right\|_{L^{1}\left(\mu_{Y}\right)}
$$

Below we write $\gamma(\tilde{P})$ to indicate that this is a positive number that depend on the operator $\tilde{P} \in\left\{\tilde{R}_{0}-\tilde{R}_{t}, \tilde{R}_{0}^{\prime}, \tilde{R}_{0}-\tilde{R}_{t}-t \tilde{R}_{0}\right\}$. Using the fact that $\tilde{R}_{0}=R$ and that $(\xi I-R)^{-1}=\sum_{j \geq 0} \xi^{-j-1} R^{j}$ and putting the above together, we obtain

$$
\begin{aligned}
& \int_{|\xi-1|=\delta}\left\|\tilde{P}(\xi I-R)^{-1} v_{Y} w\right\|_{\mathcal{B}_{0}} d \xi \leq \int_{|\xi-1|=\delta}\left\|\xi^{-1} \tilde{P}\left(v_{Y} w-\mu_{Y}\left(v_{Y} w\right)\right)\right\|_{\mathcal{B}_{0}} d \xi \\
& \quad+\int_{|\xi-1|=\delta}\left\|\sum_{j \geq 1} \xi^{-j-1} \tilde{P} R^{j}\left(v_{Y} w-\mu_{Y}\left(v_{Y} w\right)\right)+(\xi-1)^{-1} \mu_{Y}\left(v_{Y} w\right) \tilde{P} 1\right\|_{\mathcal{B}_{0}} d \xi \\
& \ll t^{\gamma(\tilde{P})}\left(\left\|\hat{\kappa}_{\sigma}\right\|_{L^{\gamma q}\left(\mu_{Y}\right)}^{\gamma}\left\|v_{Y}\right\|_{L^{b}\left(\mu_{Y}\right)}\|w\|_{\mathcal{B}_{0}}\right. \\
& \left.\quad+\sum_{j \geq 1} \int_{|\xi-1|=\delta}|\xi|^{-j-1}\left\|R^{j}\left(v_{Y} w-\mu_{Y}\left(v_{Y} w\right)\right)\right\|_{\mathcal{B}_{0}} d \xi\right) \\
& \ll t^{\gamma(\tilde{P})}\left(\left\|\hat{\kappa}_{\sigma}\right\|_{L^{\gamma q}\left(\mu_{Y}\right)}^{\gamma}\left\|v_{Y}\right\|_{L^{b}\left(\mu_{Y}\right)}\|w\|_{\mathcal{B}_{0}}\right) \\
& \left.+\sum_{j \geq 1} \int_{|\xi-1|=\delta}|\xi|^{-j-1} \rho^{j}\left\|v_{Y}\right\|_{L^{1}\left(\mu_{Y}\right)}\|w\|_{\mathcal{B}_{0}} d \xi\right) .
\end{aligned}
$$


due to Lemma 5.9 and to $(50)$, with $\gamma\left(\tilde{R}_{0}-\tilde{R}_{t}\right)=1, \gamma\left(\tilde{R}_{0}^{\prime}\right)=0, \gamma\left(\tilde{R}_{0}-\tilde{R}_{t}-t \tilde{R}_{0}^{\prime}\right)=\gamma$. So

$$
\begin{aligned}
& \int_{|\xi-1|=\delta}\left\|\tilde{P}(\xi I-R)^{-1}\left(v_{Y} w\right)\right\|_{\mathcal{B}_{0}} d \xi \ll t^{\gamma(\tilde{P})}\left\|\hat{\kappa}_{\sigma}\right\|_{L^{\gamma q}\left(\mu_{Y}\right)}^{\gamma}\left\|v_{Y}\right\|_{L^{b}\left(\mu_{Y}\right)}\|w\|_{\mathcal{B}_{0}} \\
& \left(1+\delta \sum_{j \geq 1} \rho^{j}(1-\delta)^{-j}\right)
\end{aligned}
$$

The claims follow by choosing $\delta$ so that $\sum_{j \geq 2} \rho^{j}|\xi|^{-j} \leq \sum_{j \geq 2} \rho^{j}(1-\delta)^{-j}<\infty$.

5.4. Expansion of $t \mapsto \int_{Y} \Pi_{t} d \mu_{Y}$. The next estimate, of independent interest, requires a more careful analysis and strongly exploits that the modulus is outside of the integral. Its proof uses arguments somewhat similar to the ones in [16] together with arguments exploiting symmetries on the tower $\Delta$. Recall that $p>2$.

Lemma 5.12. Let $b \in(p,+\infty]$. Let $c_{0}(v, w)$ be as defined in Proposition 5.4, namely

$c_{0}(v w)=i\left(\sum_{j \geq 0} \int_{\Delta} \hat{\kappa} \circ f^{j} . v w d \mu_{\Delta}+\int_{\Delta} v w d \mu_{\Delta} \sum_{j \geq 0} \int_{\Delta} \hat{\kappa} \frac{1_{Y} \circ f^{j+1}}{\mu_{\Delta}(Y)} d \mu_{\Delta}\right) \in \mathbb{C}^{2}$.

Then for every $(v, w)$ as in Proposition 5.3 and for every $\gamma \in\left(1,2 \frac{p-1}{p}\right)$,

$$
\left|\int_{Y}\left(\Pi_{t}-\Pi_{0}\right)(v w) d \mu_{Y}-c_{0}(v w) \cdot t\right| \ll|t|^{\gamma}\left(\|v w\|_{\mathcal{B}}+\|w\|_{\mathcal{B}_{0}}\|v\|_{L^{b}\left(\mu_{\Delta}\right)}\right) \text { as } t \rightarrow 0 .
$$

Proof. Let $\gamma \in\left(1,2 \frac{p-1}{p}\right)$, i.e. $1<\gamma<2\left(1-\frac{1}{p}\right)<2\left(1-\frac{1}{b}\right)$. Fix $\varepsilon>0$ so that $\gamma+\varepsilon<2\left(1-\frac{1}{p}\right)<2$. Fix $1<q<\frac{2 b}{b+2}$ close enough to 1 so that $1<$ $\gamma+\varepsilon<2\left(\frac{1}{q}-\frac{1}{b}\right)$. Let $p_{1} \in(1,2)$. Consider $\theta \in(0,1)$ satisfying (16) and such that $\theta_{1}^{\frac{\left(p_{1}-1\right)}{p_{1}}}<\theta$. Set $\theta_{0}:=\sqrt{\theta}$ and $r:=\theta_{0}^{1 / \vartheta}$ with $\vartheta>2$ large enough (i.e. $r$ close enough to 1$)$ so that

$$
\frac{2(\gamma+\varepsilon)}{\vartheta-1}<\varepsilon \text { and } \frac{(\gamma+\varepsilon)(\vartheta-2)}{\vartheta-1}>\gamma-1
$$

We choose $\delta$ small enough so that $(1-\delta)^{-\frac{p}{p-1}} \theta_{1}<1,(1-\delta)^{-\frac{b}{b-1}} \theta_{1}^{\frac{b}{b-1}\left(\frac{1}{p}-\frac{1}{b}\right)}<1$,

$$
\delta+r<1, \quad(1-\delta)^{-1} \rho<1, \quad(1-\delta)^{-1} \theta_{0}<1
$$

and so that $\Pi_{t}=\int_{|\xi-1|=\delta}\left(\xi I-P_{t}\right)^{-1} d \xi$ for every $t$ in a small neighbourhood of 0 (this is possible thanks to [16] since 1 is isolated in the spectrum of $\left.P_{0}\right)$. We will make use of this choice from equation (66) onwards. Recall that $\left(\xi I-P_{t}\right)^{-1}-\left(\xi I-P_{0}\right)^{-1}=$ $\left(\xi I-P_{0}\right)^{-1}\left(P_{t}-P_{0}\right)\left(\xi I-P_{t}\right)^{-1}$, and so

$$
\int_{Y}\left(\Pi_{t}-\Pi_{0}\right)(v w) d \mu_{Y}=\frac{1}{2 i \pi} \int_{Y} \int_{|\xi-1|=\delta}\left(\xi I-P_{0}\right)^{-1}\left(P_{t}-P_{0}\right)\left(\xi I-P_{t}\right)^{-1}(v w) d \xi d \mu_{Y}
$$




$$
\begin{aligned}
& =I_{1}(t)+I_{2}(t)=\frac{1}{2 i \pi} \int_{Y} \int_{|\xi-1|=\delta}\left(\xi I-P_{0}\right)^{-1}\left(P_{t}-P_{0}\right)\left(\xi I-P_{0}\right)^{-1}(v w) d \xi d \mu_{Y} \\
& +\frac{1}{2 i \pi} \int_{Y} \int_{|\xi-1|=\delta}\left(\xi I-P_{0}\right)^{-1}\left(P_{t}-P_{0}\right)\left(\left(\xi I-P_{t}\right)^{-1}-\left(\xi I-P_{0}\right)^{-1}\right)(v w) d \xi d \mu_{Y} .
\end{aligned}
$$

- Let us start with the computation of $I_{1}(t)$. Setting $N^{\prime}:=\left\lfloor(\gamma+\varepsilon) \log t / \log \theta_{0}\right\rfloor$,

$$
\begin{aligned}
I_{1}(t)= & \frac{1}{2 i \pi} \int_{Y} \int_{|\xi-1|=\delta}\left(\xi I-P_{0}\right)^{-1}\left(P_{t}-P_{0}\right)\left(\xi I-P_{0}\right)^{-1}(v w) d \xi d \mu_{Y} \\
= & I_{1, N^{\prime}}(t)+I_{1, N^{\prime}}^{\prime}(t)=\frac{1}{2 i \pi} \sum_{j=0}^{N^{\prime}-1} \int_{Y} \int_{|\xi-1|=\delta} \xi^{-j-1} P_{0}^{j}\left(P_{t}-P_{0}\right)\left(\xi I-P_{0}\right)^{-1}(v w) d \xi d \mu_{Y} \\
& +\frac{1}{2 i \pi} \int_{Y} \int_{|\xi-1|=\delta} \xi^{-N^{\prime}}\left(\xi I-P_{0}\right)^{-1} P_{0}^{N^{\prime}}\left(P_{t}-P_{0}\right)\left(\xi I-P_{0}\right)^{-1}(v w) d \xi d \mu_{Y}
\end{aligned}
$$

Observe that $|\xi-1|=\delta$ implies $\left|\xi^{-1}\right| \leq(1-\delta)^{-1}<r^{-1}$. Due to Lemma 5.2,

$$
\begin{gathered}
\left|I_{1, N^{\prime}}(t)-\frac{1}{2 i \pi} \sum_{j=0}^{N^{\prime}-1} \int_{Y} \int_{|\xi-1|=\delta} \xi^{-j-1} P_{0}^{j}\left(t \cdot P_{0}^{\prime}\right)\left(\xi I-P_{0}\right)^{-1}(v w) d \xi d \mu_{Y}\right| \\
\ll r^{-N^{\prime}}\left\|\left(P_{t}-P_{0}-t \cdot P_{0}^{\prime}\right)\left(\xi I-P_{0}\right)^{-1}(v w)\right\|_{L^{1}(\Delta)} \ll r^{-N^{\prime}} t^{\gamma+\varepsilon}\|v w\|_{\mathcal{B}},
\end{gathered}
$$

since our assumption on $\varepsilon, q$ ensures that $\gamma+\varepsilon<2\left(\frac{1}{q}-\frac{1}{p}\right)$. Therefore $\left|I_{1, N^{\prime}}(t)-\frac{1}{2 \pi} \sum_{j=0}^{N^{\prime}-1} \int_{f^{-j-1} Y} \int_{|\xi-1|=\delta} \xi^{-j-1}(t \cdot \kappa)\left(\xi I-P_{0}\right)^{-1}(v w) d \xi d \mu_{Y}\right| \ll r^{-N^{\prime}} t^{\gamma+\varepsilon}\|v w\|_{\mathcal{B}}$.

Moreover, since $\sup _{|\xi-1|=\delta}\left\|\left(\xi I-P_{0}\right)^{-1}\right\|_{\mathcal{B}}<\infty$, using the Fubini theorem, we obtain

$$
\begin{aligned}
& I_{1, N^{\prime}}^{\prime}(t)=\frac{1}{2 i \pi} \int_{Y} \int_{|\xi-1|=\delta} \xi^{-N^{\prime}}\left(\xi I-P_{0}\right)^{-1}\left(\Pi_{0}+N_{0}^{N^{\prime}}\right)\left(P_{t}-P_{0}\right)\left(\xi I-P_{0}\right)^{-1}(v w) d \xi d \mu_{Y} \\
& =\frac{1}{2 i \pi \mu_{\Delta}(Y)} \int_{|\xi-1|=\delta} \xi^{-N^{\prime}} \Pi_{0}\left(1_{Y}\left(\xi I-P_{0}\right)^{-1} 1\right) \Pi_{0}\left(\left(P_{t}-P_{0}\right)\left(\xi I-P_{0}\right)^{-1}(v w)\right) d \xi+O\left(r^{-N^{\prime}} \theta^{N^{\prime}}\right) \\
& =\frac{1}{2 \pi \mu_{\Delta}(Y)} \int_{|\xi-1|=\delta} \xi^{-N^{\prime}} \Pi_{0}\left(1_{Y}\left(\xi I-P_{0}\right)^{-1} 1\right) \Pi_{0}\left(t \cdot \hat{\kappa}\left(\xi I-P_{0}\right)^{-1}(v w)\right) d \xi \\
& \quad+O\left(\left(r^{-N^{\prime}}|t|^{\gamma+\varepsilon}+r^{-N^{\prime}} \theta^{N^{\prime}}\right)\|v w\|_{\mathcal{B}}\right),
\end{aligned}
$$

where we used again Lemma 5.2. Since $r=\theta_{0}^{1 / \vartheta}, \vartheta>2$ and $\theta=\theta_{0}^{1 / 2}$, we obtain

$$
\begin{aligned}
I_{1}(t)= & \frac{1}{2 \pi} t \cdot A_{1, N^{\prime}}(t)+O\left(\left(r^{-N^{\prime}} t^{\gamma+\varepsilon}+\theta_{0}^{\frac{(\vartheta-2) N^{\prime}}{2}}\right)\|v w\|_{\mathcal{B}}\right), \\
A_{1, N^{\prime}}(t):= & U_{N^{\prime}}(t)+V_{N^{\prime}}(t)=\sum_{j=0}^{N^{\prime}-1} \int_{Y} \int_{|\xi-1|=\delta} \xi^{-j-1} P^{j+1}\left(\hat{\kappa}\left(\xi I-P_{0}\right)^{-1}(v w)\right) d \xi d \mu_{Y} \\
& +\frac{1}{2 \pi \mu_{\Delta}(Y)} \int_{|\xi-1|=\delta} \xi^{-N^{\prime}} \Pi_{0}\left(1_{Y}\left(\xi I-P_{0}\right)^{-1} 1\right) \Pi_{0}\left(\hat{\kappa}\left(\xi I-P_{0}\right)^{-1}(v w)\right) d \xi
\end{aligned}
$$


Due to our choice of $N^{\prime}$ and since $\theta_{0}=r^{\vartheta}$, we have

$$
r^{N^{\prime} \vartheta}=\theta_{0}^{N^{\prime}} \ll t^{\gamma+\varepsilon}
$$

so $r^{-N^{\prime}} \ll t^{-\frac{\gamma+\varepsilon}{\vartheta}} \ll t^{-\varepsilon}$, due to our assumptions on $\vartheta$. Note that

$$
\left(\xi I-P_{0}\right)^{-1}(v w)=\sum_{j \geq 0} \xi^{-j-1} P_{0}^{j}(v w) \text { and }\left(\xi I-P_{0}\right)^{-1} 1_{\Delta}=(\xi-1)^{-1} 1_{\Delta}
$$

and so $V_{N^{\prime}}(t)=\frac{1}{\mu_{\Delta}(Y)} \int_{|\xi-1|=\delta} \sum_{j \geq 0} \xi^{-N^{\prime}-j-1}(\xi-1)^{-1} \mu_{\Delta}(Y) \Pi_{0}\left(\hat{\kappa} P_{0}^{j}(v w)\right) d \xi$. Thus

$$
V_{N^{\prime}}(t)=\frac{1}{\mu_{\Delta}(Y)} \sum_{j \geq 0} \int_{|\xi-1|=\delta} \xi^{-N^{\prime}-j-1}(\xi-1)^{-1} \mu_{\Delta}(Y) \Pi_{0}\left(\hat{\kappa} N_{0}^{j}(v w)\right) d \xi
$$

since $P_{0}^{j}=\mu_{\Delta}(\cdot) 1_{\Delta}+N_{0}^{j}$, since $\Pi_{0}(\hat{\kappa})=0$ and since $\sum_{j \geq 1}\left|\xi^{-j} \Pi_{0}\left(\hat{\kappa} N_{0}^{j}(v w)\right)\right| \ll$ $\sum_{j \geq 1}\left|(1-\delta)^{-1} \theta\right|^{j}\|\hat{\kappa}\|_{L^{p /(p-1)}\left(\mu_{\Delta}\right)}\|v w\|_{\mathcal{B}}<\infty$, due to our choice of $\delta$. Therefore, due to the Cauchy integral formula,

$$
V_{N^{\prime}}(t)=2 i \pi \sum_{j \geq 0} \Pi_{0}\left(\hat{\kappa} N_{0}^{j}(v w)\right)=2 i \pi \sum_{j \geq 0} \int_{\Delta} \hat{\kappa} \circ f^{j} v w d \mu_{\Delta} .
$$

Combining this with (55) and (56), we obtain

$$
I_{1}(t)=t \cdot\left(\frac{1}{2 \pi} U_{N^{\prime}}(t)+i \sum_{j \geq 0} \int_{\Delta} \hat{\kappa} \circ f^{j} . v w d \mu_{\Delta}\right)+O\left(|t|^{\gamma}\|v w\|_{\mathcal{B}}\right) .
$$

We compute that

$$
\begin{aligned}
& U_{N^{\prime}}(t)=\frac{1}{\mu_{\Delta}(Y)} \sum_{j=0}^{N^{\prime}-1} \int_{|\xi-1|=\delta} \xi^{-j-1} \int_{\Delta} 1_{Y} P^{j+1}\left(\hat{\kappa}\left(\xi I-P_{0}\right)^{-1}(v w)\right) d \mu_{\Delta} d \xi \\
& =\frac{1}{\mu_{\Delta}(Y)} \sum_{j=0}^{N^{\prime}-1} \int_{|\xi-1|=\delta} \xi^{-j-1} \int_{\Delta} \hat{\kappa}\left(\xi I-P_{0}\right)^{-1}(v w) 1_{Y} \circ f^{j+1} d \mu_{\Delta} d \xi \\
& =\frac{U_{1, N^{\prime}}(t)+U_{2, N^{\prime}}(t)}{\mu_{\Delta}(Y)}, \\
& U_{1, N^{\prime}}(t)=\sum_{j=0}^{N^{\prime}-1} \int_{|\xi-1|=\delta} \xi^{-j-1} \int_{\Delta} \hat{\kappa}\left(\xi I-P_{0}\right)^{-1}\left(v w-\mu_{\Delta}(v w)\right) 1_{Y} \circ f^{j+1} d \mu_{\Delta} d \xi, \\
& \left.U_{2, N^{\prime}}(t)\right)=\mu_{\Delta}(v w) \sum_{j=0}^{N^{\prime}-1} \int_{|\xi-1|=\delta} \xi^{-j-1}(\xi-1)^{-1} \int_{\Delta} \hat{\kappa} 1_{Y} \circ f^{j+1} d \mu_{\Delta} d \xi, \quad(60)
\end{aligned}
$$

since $\left(\xi I-P_{0}\right)^{-1} 1_{\Delta}=(\xi-1)^{-1} 1_{\Delta}$. Due to the Cauchy integral formula,

$$
U_{2, N^{\prime}}(t):=2 i \pi \mu_{\Delta}(v w) \sum_{j=0}^{N^{\prime}-1} \int_{\Delta} \hat{\kappa} 1_{Y} \circ f^{j+1} d \mu_{\Delta}
$$




$$
\begin{aligned}
& =2 i \pi \mu_{\Delta}(v w) \sum_{j \geq 0} \int_{\Delta} \hat{\kappa} 1_{Y} \circ f^{j+1} d \mu_{\Delta}+O\left(\theta_{0}^{N^{\prime}}\|v w\|_{L^{1}\left(\mu_{\Delta}\right)}\right) \\
& =2 i \pi \mu_{\Delta}(v w) \sum_{j \geq 0} \int_{\Delta} \hat{\kappa} 1_{Y} \circ f^{j+1} d \mu_{\Delta}+O\left(|t|^{\gamma}\|v w\|_{\mathcal{B}}\right),
\end{aligned}
$$

where in the penultimate line we have used that $\int_{\Delta} \hat{\kappa} 1_{Y} \circ f^{j} d \bar{\mu}_{\Delta}=O\left(\theta_{0}^{j}\right)$ (see Lemma C.2 below) and in the last line we have used (57). Next, we estimate $U_{1, N^{\prime}}$ defined in (60). Since $\left.v\right|_{(a, \ell)}=C_{(a, \ell)}$ where $C_{(a, \ell)}$ are constants, $U_{1, N^{\prime}}(t)$ is equal to the following quantities

$$
\begin{aligned}
= & \sum_{j=0}^{N^{\prime}} \int_{|\xi-1|=\delta} \xi^{-j-1} \int_{\Delta} \sum_{r \geq 0} \xi^{-r-1} P_{0}^{r} \sum_{(a, \ell)}\left(v w 1_{(a, \ell)}-\int_{(a, l)} v w d \mu_{\Delta}\right)\left(\hat{\kappa} 1_{Y} \circ f^{j}\right) d \mu_{\Delta} d \xi \\
= & \sum_{j=0}^{N^{\prime}} \int_{|\xi-1|=\delta} \xi^{-j-1} \sum_{r \geq 0} \xi^{-r-1} \sum_{(a, \ell)} \int_{\Delta} P_{0}^{r}\left(v w 1_{(a, \ell)}-\int_{(a, l)} v w d \mu_{\Delta}\right)\left(\hat{\kappa} 1_{Y} \circ f^{j}\right) d \mu_{\Delta} d \xi \\
= & \sum_{j=0}^{N^{\prime}} \int_{|\xi-1|=\delta} \xi^{-j-1} \sum_{r \geq 0} \xi^{-r-1} \sum_{(a, \ell)} C_{(a, \ell)} \int_{\Delta}\left(w 1_{(a, \ell)}-\mu_{\Delta}\left(w 1_{(a, l)}\right)\right)\left(\hat{\kappa} 1_{Y} \circ f^{j}\right) \circ f^{r} d \mu_{\Delta} d \xi \\
= & \sum_{r \geq 0} \sum_{(a, \ell)} \sum_{j=0}^{N^{\prime}} C_{(a, \ell)} \int_{\Delta}\left(w 1_{(a, \ell)}-\mu_{\Delta}\left(w 1_{(a, l)}\right)\left(\hat{\kappa} 1_{Y} \circ f^{j}\right) \circ f^{r} d \mu_{\Delta} \int_{|\xi-1|=\delta} \xi^{-j-r-2} d \xi\right.
\end{aligned}
$$

and thus $U_{1, N^{\prime}}(t)=0$. The interchange of sums and integrals in the above equations is justified by Lemma C.3. This is the only part of the proof where this assumption that is crucially used. Combining (59), (60), (61) and $U_{1, N^{\prime}}(t)=0$, we obtain that

$$
\begin{aligned}
I_{1}(t)= & i t \cdot\left(\sum_{j \geq 0} \int_{\Delta} \hat{\kappa} \circ f^{j} \cdot v w d \mu_{\Delta}+\int_{\Delta} v w d \mu_{\Delta} \sum_{j \geq 0} \int_{\Delta} \hat{\kappa} \frac{1_{Y} \circ f^{j+1}}{\mu_{\Delta}(Y)} d \bar{\mu}_{\Delta}\right) \\
& +O\left(t^{\gamma}\left(\|v w\|_{\mathcal{B}}+\|w\|_{\mathcal{B}_{0}}\|v\|_{L^{b}\left(\mu_{\Delta}\right)}\right)\right) .
\end{aligned}
$$

- Let us prove that $\left|I_{2}(t)\right| \ll|t|^{\gamma}\|v w\|_{\mathcal{B}}$. This will give the conclusion. First, compute that for any $N \geq 1$,

$$
\begin{aligned}
I_{2}(t)= & \int_{Y} \int_{|\xi-1|=\delta}\left(\xi I-P_{0}\right)^{-1}\left(P_{t}-P_{0}\right)\left(\xi I-P_{0}\right)^{-1}\left(P_{t}-P_{0}\right)\left(\xi I-P_{t}\right)^{-1}(v w) d \xi d \mu_{Y} \\
= & \sum_{j=0}^{N-1} \int_{|\xi-1|=\delta} \xi^{-j-1} \int_{Y} P_{0}^{j}\left(P_{t}-P_{0}\right)\left(\xi I-P_{0}\right)^{-1}\left(P_{t}-P_{0}\right)\left(\xi I-P_{t}\right)^{-1}(v w) d \mu_{Y} d \xi \\
& +\int_{Y} \int_{|\xi-1|=\delta} \xi^{-N}\left(\xi I-P_{0}\right)^{-1} P_{0}^{N}\left(P_{t}-P_{0}\right)\left(\xi I-P_{0}\right)^{-1}\left(P_{t}-P_{0}\right)\left(\xi I-P_{t}\right)^{-1}(v w) d \xi d \mu_{Y} \\
= & S_{N}(t)+E_{N}(t) .
\end{aligned}
$$

We further decompose $S_{N}$ and $E_{N}$, choosing $N:=\left\lfloor(\gamma+\varepsilon) \frac{\log t}{\log \left(\theta_{0} / r\right)}\right\rfloor$ to prove the claim. We first deal with $S_{N}$.

$$
S_{N}(t)=\sum_{j=0}^{N-1} \sum_{\ell=0}^{N-1} \int_{|\xi-1|=\delta} \xi^{-j-1} \xi^{-\ell-1} \int_{Y} P_{0}^{j}\left(P_{t}-P_{0}\right) P_{0}^{\ell}\left(P_{t}-P_{0}\right)\left(\xi I-P_{t}\right)^{-1}(v w) d \mu_{Y} d \xi
$$




$$
\begin{aligned}
& +\sum_{j=0}^{N-1} \int_{Y} \int_{|\xi-1|=\delta} \xi^{-j-1} P_{0}^{j}\left(P_{t}-P_{0}\right)\left(\xi I-P_{0}\right)^{-1} \xi^{-N} P_{0}^{N}\left(P_{t}-P_{0}\right)\left(\xi I-P_{t}\right)^{-1}(v w) d \xi d \mu_{Y} \\
= & S_{N, N}(t)+E_{N, N}(t) .
\end{aligned}
$$

Set $\gamma_{0}=\frac{\gamma+\varepsilon}{2} \in(0,1)$. Note that $\gamma+\varepsilon<2\left(1-\frac{1}{p}\right)$ implies that $\frac{p}{p-1} \gamma_{0}<1$.

$$
\begin{aligned}
\mid \int_{Y} P_{0}^{j} & \left(P_{t}-P_{0}\right) P_{0}^{\ell}\left(P_{t}-P_{0}\right)\left(\xi I-P_{t}\right)^{-1}(v w) d \mu_{Y} \mid \\
& =\left|\int_{Y}\left(e^{i t \cdot \hat{\kappa}}-1\right) P_{0}^{\ell}\left(P_{t}-P_{0}\right)\left(\xi I-P_{t}\right)^{-1}(v w) d \mu_{Y}\right| \\
& \ll\left\|\left(\xi I-P_{t}\right)^{-1}(v w)\right\|_{\mathcal{B}}\left\|\left(\left(e^{i t \cdot \hat{\kappa}}-1\right) 1_{Y}\right) \circ f^{\ell+1}\left(e^{i t \cdot \hat{\kappa}}-1\right)\right\|_{L^{\frac{p}{p-1}}\left(\mu_{\Delta}\right)} \\
& \ll|t|^{2 \gamma_{0}}\|\hat{\kappa}\|^{2 \gamma_{0}}{ }_{L}^{2 \gamma_{0} \frac{p}{p-1}}\|v w\|_{\mathcal{B}},
\end{aligned}
$$

where we have used the Hölder inequality combined with $\mathcal{B} \hookrightarrow L^{p}\left(\mu_{\Delta}\right)$ and finally, in the last line, we used the inequality $\left|e^{i t \cdot \hat{\kappa}}-1\right| \ll|t \hat{\kappa}|^{\gamma_{0}}$ combined with the CauchySchwarz inequality. Hence, using the fact that $|\xi|^{-1} \leq(1-\delta)^{-1} \leq r^{-1}$,

$$
\left|S_{N, N}(t)\right| \ll r^{-2 N}|t|^{2 \gamma_{0}}=r^{-2 N}|t|^{\gamma+\varepsilon} .
$$

By (15), we have $P_{0}^{N}=\Pi_{0}+N_{0}^{N}$ with $\left\|N_{0}^{N}\right\|_{\mathcal{B}} \ll \theta_{0}^{N}$. Hence,

$$
\begin{aligned}
& E_{N, N}(t) \\
& =\sum_{j=0}^{N-1} \int_{Y} \int_{|\xi-1|=\delta} \xi^{-j-1} P_{0}^{j}\left(P_{t}-P_{0}\right) \xi^{-N}\left(\xi I-P_{0}\right)^{-1} \Pi_{0}\left(\left(P_{t}-P_{0}\right)\left(\xi I-P_{t}\right)^{-1}(v w)\right) d \xi d \mu_{Y} \\
& +\sum_{j=0}^{N-1} \int_{Y} \int_{|\xi-1|=\delta} \xi^{-j-1} P_{0}^{j}\left(P_{t}-P_{0}\right) \xi^{-N}\left(\xi I-P_{0}\right)^{-1} N_{0}^{N}\left(\left(P_{t}-P_{0}\right)\left(\xi I-P_{t}\right)^{-1}(v w)\right) d \xi d \mu_{Y} \\
& =E_{N, N}^{1}(t)+E_{N, N}^{2}(t) .
\end{aligned}
$$

But for any $\xi$ so that $|\xi-1|=\delta$,

$$
\begin{aligned}
\left|\Pi_{0}\left(\left(P_{t}-P_{0}\right)\left(\xi I-P_{t}\right)^{-1}(v w)\right)\right| & \ll \int_{\Delta}\left|\left(P_{t}-P_{0}\right)\left(\xi I-P_{t}\right)^{-1}(v w)\right| d \mu_{\Delta} \\
& \ll\left\|P_{t}-P_{0}\right\|_{\mathcal{B} \rightarrow L^{1}}\|v w\|_{\mathcal{B}} \ll|t|\|v w\|_{\mathcal{B}} .
\end{aligned}
$$

Hence,

$$
\left|E_{N, N}^{1}(t)\right| \ll|t|\|v w\|_{\mathcal{B}} \sum_{j=0}^{N-1} \int_{\Delta} \int_{|\xi-1|=\delta}\left|\xi^{-j-1}\right|\left|\xi^{-N}\right|\left|\left(P_{t}-P_{0}\right)\left(\xi I-P_{0}\right)^{-1}\left(1_{Y}\right)\right| d \xi d \mu_{\Delta} .
$$

The above together with (52) implies that

$$
\left|E_{N, N}^{1}(t)\right| \ll|t| r^{-2 N}\left\|P_{t}-P_{0}\right\|_{\mathcal{B} \rightarrow L^{1}}\|v w\|_{\mathcal{B}} \ll|t|^{2} r^{-2 N}\|v w\|_{\mathcal{B}} .
$$

Recall that $\left\|N_{0}^{N}\right\|_{\mathcal{B}} \ll \theta_{0}^{N}$. This together with (52) implies that

$$
\left|E_{N, N}^{2}(t)\right| \ll\left(\theta_{0} / r^{2}\right)^{N}\left\|P_{t}-P_{0}\right\|_{\mathcal{B} \rightarrow L^{1}}\|v w\|_{\mathcal{B}} \ll|t|\left(\theta_{0} / r^{2}\right)^{N}\|v w\|_{\mathcal{B}} .
$$


This together with (63), (65), (66) and (67) implies that

$$
\left|S_{N}(t)\right| \ll\left(r^{-2 N}|t|^{\gamma+\varepsilon}+|t|^{2} r^{-2 N}+|t|\left(\theta_{0} / r^{2}\right)^{N}\right)\|v w\|_{\mathcal{B}} .
$$

Proceeding as above in estimating $E_{N, N}(t)$, we compute that

$$
\begin{aligned}
E_{N}(t)= & E_{N}^{1}(t)+E_{N}^{2}(t) \\
= & \int_{Y} \int_{|\xi-1|=\delta} \xi^{-N}\left(\xi I-P_{0}\right)^{-1} \Pi_{0}\left(\left(P_{t}-P_{0}\right)\left(\xi I-P_{0}\right)^{-1}\left(P_{t}-P_{0}\right)\left(\xi I-P_{t}\right)^{-1}(v w)\right) d \xi d \mu_{Y} \\
& +\int_{Y} \int_{|\xi-1|=\delta} \xi^{-N}\left(\xi I-P_{0}\right)^{-1} N_{0}^{N}\left(\left(P_{t}-P_{0}\right)\left(\xi I-P_{0}\right)^{-1}\left(P_{t}-P_{0}\right)\left(\xi I-P_{t}\right)^{-1}(v w)\right) d \xi d \mu_{Y} .
\end{aligned}
$$

Similar to (67), $\left|E_{N}^{2}(t)\right| \ll\left(\theta_{0} / r\right)^{N}\|v w\|_{\mathcal{B}}$. We need to decompose $E_{N}^{1}(t)$ further. To start we reduce the analysis to $\Pi_{0}\left(\left(P_{t}-P_{0}\right)\left(\xi I-P_{0}\right)^{-1}\left(P_{t}-P_{0}\right)\left(\xi I-P_{t}\right)^{-1}(v w)\right)$, for $\xi$ so that $|\xi-1|=\delta$. With this specified we compute that for any $L \geq 1$,

$$
\begin{aligned}
& \Pi_{0}\left(\left(P_{t}-P_{0}\right)\left(\xi I-P_{0}\right)^{-1}\left(P_{t}-P_{0}\right)\left(\xi I-P_{t}\right)^{-1}(v w)\right) \\
&=\int_{Y}\left(P_{t}-P_{0}\right)\left(\xi I-P_{0}\right)^{-1}\left(P_{t}-P_{0}\right)\left(\xi I-P_{t}\right)^{-1}(v w) d \mu \\
&= S_{L}^{\prime}(t)+E_{L}^{\prime}(t)=\sum_{j=0}^{L-1} \xi^{-j-1} \\
& \int_{Y}\left(P_{t}-P_{0}\right) P_{0}^{j}\left(P_{t}-P_{0}\right)\left(\xi I-P_{t}\right)^{-1}(v w) d \mu \\
&+\int_{Y}\left(P_{t}-P_{0}\right)\left(\xi I-P_{0}\right)^{-1} \xi^{-L} P^{L}\left(P_{t}-P_{0}\right)\left(\xi I-P_{t}\right)^{-1}(v w) d \mu .
\end{aligned}
$$

Due to (64), $\left|S_{L}^{\prime}(t)\right| \ll r^{-L}|t|^{\gamma+\varepsilon}\|v w\|_{\mathcal{B}}$. Next, to estimate $E_{L}^{\prime}(t)$, we repeat the argument used in estimating $E_{N, N}(t)$ above. Namely,

$$
\begin{aligned}
\left|E_{L}^{\prime}(t)\right| & \ll\left|\int_{Y}\left(P_{0}-P_{t}\right)\left(\xi I-P_{0}\right)^{-1} \xi^{-L} \Pi_{0}\left(P_{t}-P_{0}\right)\left(\xi I-P_{t}\right)^{-1}(v w) d \mu\right| \\
& +\left|\int_{Y}\left(P_{0}-P_{t}\right)\left(\xi I-P_{0}\right)^{-1} \xi^{-L} N_{0}^{L}\left(P_{t}-P_{0}\right)\left(\xi I-P_{t}\right)^{-1}(v w) d \mu\right| \\
& \ll\left(r^{-L}|t|^{2}+|t|\left(\theta_{0} / r\right)^{L}\right)\|v w\|_{\mathcal{B}} .
\end{aligned}
$$

Therefore $\left|E_{N}(t)\right| \ll\left(\theta_{0} / r\right)^{N}+r^{-N}\left(r^{-L}|t|^{\gamma+\varepsilon}+|t|\left(\theta_{0} / r\right)^{L}\right)\|v w\|_{\mathcal{B}}$. Gathering this and (68), we obtain

$$
\left|I_{2}(t)\right| \ll\left(r^{-2 N}|t|^{\gamma+\varepsilon}+|t|\left(\theta_{0} / r^{2}\right)^{N}+\left(\theta_{0} / r\right)^{N}+r^{-N}\left(r^{-L}|t|^{\gamma+\varepsilon}+|t|\left(\theta_{0} / r\right)^{L}\right)\right)\|v w\|_{\mathcal{B}} .
$$

Recall that $N:=\left\lfloor(\gamma+\varepsilon) \frac{\log |t|}{\log \left(\theta_{0} / r\right)}\right\rfloor$, so that $\left(\theta_{0} / r\right)^{N} \sim|t|^{\gamma+\varepsilon}$. Recall also that $\theta_{0}=r^{\vartheta}$ with $\vartheta>2$ so that $\frac{2(\gamma+\varepsilon)}{\vartheta-1}<\varepsilon$ and $\frac{(\gamma+\varepsilon)(\vartheta-2)}{\vartheta-1}>\gamma-1$. Hence $\theta_{0} / r=r^{\vartheta-1}$ and $r^{-N} \sim|t|^{-\frac{\gamma+\varepsilon}{\vartheta-1}}$ and $\left(\theta_{0} / r^{2}\right)^{N}=r^{N(\vartheta-2)} \approx|t|^{\frac{(\gamma+\varepsilon)(\vartheta-2)}{\vartheta-1}}$ thus

$\left|I_{2}(t)\right| \ll\left(|t|^{\gamma+\varepsilon-2 \frac{\gamma+\varepsilon}{\vartheta-1}}+|t|^{1+\frac{(\gamma+\varepsilon)(\vartheta-2)}{\vartheta-1}}+|t|^{\gamma+\varepsilon}+|t|^{-\frac{\gamma+\varepsilon}{\vartheta-1}}\left(r^{-L}|t|^{\gamma+\varepsilon}+|t|\left(\theta_{0} / r\right)^{L}\right)\right)\|v w\|_{\mathcal{B}}$ 


$$
\ll\left(|t|^{\gamma}+|t|^{-\frac{\gamma+\varepsilon}{\vartheta-1}}\left(\left(\theta_{0} / r\right)^{-\frac{L}{\vartheta-1}}|t|^{\gamma+\varepsilon}+|t|\left(\theta_{0} / r\right)^{L}\right)\right)\|v w\|_{\mathcal{B}},
$$

due to our choice of $\vartheta$. Now, choose $L:=\left\lfloor\frac{(\gamma+\varepsilon-1) \log |t|}{\log \left(\theta_{0} / r\right)}\right\rfloor$, so, due to our choice of $\vartheta$,

$$
\left|I_{2}(t)\right| \ll\left(|t|^{\gamma}+|t|^{-\frac{\gamma+\varepsilon}{\vartheta-1}}\left(|t|^{\gamma+\varepsilon-\frac{\gamma+\varepsilon-1}{\vartheta-1}}+|t|^{\gamma+\varepsilon}\right)\right)\|v w\|_{\mathcal{B}} \ll|t|^{\gamma}\|v w\|_{\mathcal{B}} .
$$

5.5. Proof of the expansion of $\Pi_{t}$ : proofs of Propositions 5.4 and 5.3. We first provide the proof of Propositions 5.4 relying on our technical Lemmas 5.11 and 5.12, and then we prove Proposition 5.3.

Proof of Proposition 5.4. We proceed as in [2, Proof of Lemma 3.14] using our estimates. Since 1 is a simple eigenvalue of $\tilde{R}_{t}$, and since $Q_{t}\left(1_{Y}\right)$ and $1_{Y} \Pi_{t}(v w)$ are both eigenfunctions belonging to $\mathcal{B}_{1}$ of $\tilde{R}_{t}$ associated to 1 , these two vectors are proportional and so,

$$
1_{Y} \Pi_{t}(v w)=\mu_{Y}\left(1_{Y} \Pi_{t}(v w)\right) \frac{Q_{t}\left(1_{Y}\right)}{\mu_{Y}\left(Q_{t}\left(1_{Y}\right)\right)}=\left(\mu_{\Delta}(v w)+t \cdot c_{0}(v w)+\varepsilon_{t}^{(1)}\right) \frac{Q_{t}\left(1_{Y}\right)}{\mu_{Y}\left(Q_{t}\left(1_{Y}\right)\right)},
$$

with $\boldsymbol{\varepsilon}_{t}^{(1)}:=\mu_{Y}\left(1_{Y}\left(\Pi_{t}-\Pi_{0}-t \cdot c_{0}\right)(v w)\right)=O\left(|t|^{\gamma}\left(\|v w\|_{\mathcal{B}}+\|w\|_{\mathcal{B}_{0}}\|v\|_{L^{b}\left(\mu_{\Delta}\right)}\right)\right)$ in $\mathbb{C}$, by Lemma 5.12. Moreover, due to Lemma 5.11, $\boldsymbol{\varepsilon}_{t}^{(2)}:=\left(Q_{t}-Q_{0}-t \cdot Q_{0}^{\prime}\right)\left(1_{Y}\right)=O\left(|t|^{\gamma}\right)$ in $\mathcal{B}_{1}$. Hence, still in $\mathcal{B}_{1}$,

$$
\begin{aligned}
\frac{Q_{t}\left(1_{Y}\right)}{\mu_{Y}\left(Q_{t}\left(1_{Y}\right)\right)} & =\frac{1_{Y}+t \cdot Q_{0}^{\prime}\left(1_{Y}\right)+\varepsilon_{t}^{(1)}}{\mu_{Y}\left(1_{Y}+t \cdot Q_{0}^{\prime}\left(1_{Y}\right)+\varepsilon_{t}^{(1)}\right)}=1_{Y}+t \cdot\left(Q_{0}^{\prime}\left(1_{Y}\right)-\mu_{Y}\left(Q_{0}^{\prime}\left(1_{Y}\right)\right) 1_{Y}\right)+O\left(|t|^{\gamma}\right) \\
& =1_{Y}+t \cdot Q_{0}^{\prime}\left(1_{Y}\right)+O\left(|t|^{\gamma}\right),
\end{aligned}
$$

since the derivation at $t=0$ of $Q_{t}^{2}\left(1_{Y}\right)=Q_{t}\left(1_{Y}\right)$ combined with $Q_{0} 1_{Y}=1_{Y}$ leads to $\mu_{Y}\left(Q_{0}^{\prime} 1_{Y}\right)=Q_{0} Q_{0}^{\prime} 1_{Y}=0$. Combining the above estimate with (70), we conclude that

$$
1_{Y} \Pi_{t}(v w)=\mu_{\Delta}(v w)+t \cdot\left(c_{0}(v w)+\mu_{\Delta}(v w) Q_{0}^{\prime}\left(1_{Y}\right)\right)+O\left(|t|^{\gamma}\left(\|v w\|_{\mathcal{B}}+\|w\|_{\mathcal{B}_{0}}\|v\|_{L^{b}\left(\mu_{\Delta}\right)}\right)\right)
$$

which leads to (36).

Remark 5.13. By the same reasoning used in obtaining $\mu_{Y}\left(Q_{0}^{\prime} 1_{Y}\right)=Q_{0} Q_{0}^{\prime} 1_{Y}=0$ with $\Pi_{0}^{\prime}$ instead of $Q_{0}^{\prime}$ and 1 instead of $1_{Y}$ we have $\mu_{\Delta}\left(\Pi_{0}^{\prime} 1\right)=0$.

Using Proposition 5.4 and equation (44) we can complete

Proof of Proposition 5.3. To simplify notations, we write $\|(v, w)\|:=\|v w\|_{\mathcal{B}}+$ $\|w\|_{\mathcal{B}_{0}}\|v\|_{L^{b}\left(\mu_{\Delta}\right)}$. Starting from (44), $\Pi_{t}(v w)(x)=\lambda_{t}^{-\omega(x)} e^{i t \cdot \hat{\kappa}_{\omega(x)} \circ \pi_{0}(x)} \Pi_{t}(v w) \circ \pi_{0}(x)$. Using the fact that $\lambda_{0}=1$ and $\lambda_{0}^{\prime}=0$, we conclude that $t \mapsto \Pi_{t}(v w)(x)$ is differentiable at 0 , with derivative

$$
\Pi_{0}^{\prime}(v w)(x)=i\left[\hat{\kappa}_{\omega(x)} \Pi_{0}(v w)\right] \circ \pi_{0}(x)+\Pi_{0}^{\prime}(v w) \circ \pi_{0}(x),
$$


where we use the definition of $1_{Y} \Pi_{0}^{\prime}$ in (36). This provides the last formula of Proposition 5.3. With this definition, since $\Pi_{0}^{\prime}$ is uniformly bounded on $Y$, for every $\eta \in(1,2)$

$$
\begin{aligned}
\left\|\Pi_{0}^{\prime}(v w)\right\|_{L^{\eta}\left(\mu_{\Delta}\right)}^{\eta} & \ll\left\|1_{Y} \Pi_{0}^{\prime}(v w)\right\|_{\infty}^{\eta}+\sum_{n \geq 1} \int_{\{\sigma>n\}}\left|\hat{\kappa}_{n}\right|^{\eta} d \mu_{Y}\|v w\|_{L^{1}\left(\mu_{Y}\right)} \\
& \ll\left(1+\sum_{n \geq 1}\left(\mu_{Y}(\sigma>n)\right)^{\frac{2-\eta}{2+\eta}}\left\|\left|\hat{\kappa}_{n}\right|^{\eta}\right\|_{L^{\frac{\eta+2}{2 \eta}}\left(\mu_{Y}\right)}\right)\|(v, w)\| \\
& \ll\left(1+\sum_{n \geq 1} \theta_{1}^{\frac{2-\eta}{2+\eta} n} n^{\eta}\|\hat{\kappa}\|_{L^{\frac{\eta+2}{2}}\left(\mu_{\Delta}\right)}^{\eta}\right)\|(v, w)\|=O(\|(v, w)\|) .
\end{aligned}
$$

Formula (71) together with (44) implies that

$$
\begin{aligned}
\left(\Pi_{t}-\right. & \left.\Pi_{0}-t \cdot \Pi_{0}^{\prime}\right)(v w)(x)=\left[\left(e^{i t \cdot \hat{\kappa}_{\omega(x)}}-1-i t \cdot \hat{\kappa}_{\omega(x)}\right) \Pi_{0}(v w)\right] \circ \pi_{0}(x) \\
+ & {\left[\left(e^{i t \hat{\kappa}_{\omega(x)}}-1\right) t \cdot \Pi_{0}^{\prime}(v w)\right] \circ \pi_{0}\left[e^{i t \cdot \hat{\kappa}_{\omega(x)}}\left(\Pi_{t}-\Pi_{0}-t \cdot \Pi_{0}^{\prime}\right)(v w)\right] \circ \pi_{0}(x) } \\
& +\left(\lambda_{t}^{-\omega(x)}-1\right)\left[e^{i t \hat{\kappa}_{\omega(x)}} \Pi_{t}(v w)\right] \circ \pi_{0}(x) .
\end{aligned}
$$

This leads to $\left\|\left(\Pi_{t}-\Pi_{0}-t \cdot \Pi_{0}^{\prime}\right)(v w)\right\|_{L^{p^{\prime}}\left(\mu_{\Delta}\right)} \leq I_{1}(t)+I_{2}(t)+I_{3}(t)+I_{4}(t)$. First

$$
\begin{aligned}
\left(I_{1}(t)\right)^{p^{\prime}} & :=\int_{\Delta}\left|e^{i t \hat{\kappa}_{\omega(x)}}-1-i t \cdot \hat{\kappa}_{\omega(x)}\right|^{p^{\prime}} \circ \pi_{0}(x) d \mu_{\Delta}(x)\left(\int_{Y}|v w| d \mu_{Y}\right)^{p^{\prime}} \\
& \ll \int_{\Delta}\left|t \cdot \hat{\kappa}_{\omega(x)} \circ \pi_{0}(x)\right|^{p^{\prime} \gamma} d \mu_{\Delta}(x)\|v w\|_{L^{1}\left(\mu_{\Delta}\right)}^{p^{\prime}} \\
& \ll \sum_{n \geq 1} \int_{\sigma>n}\left|t \cdot \hat{\kappa}_{n}\right|^{p^{\prime} \gamma} d \mu_{Y}\|v w\|_{L^{1}\left(\mu_{\Delta}\right)}^{p^{\prime}}
\end{aligned}
$$

Thus $\left(I_{1}(t)\right)^{p^{\prime}} \ll \sum_{n \geq 1}|t|^{p^{\prime} \gamma} \mu_{Y}(\sigma \geq n)^{\frac{2-\gamma}{2+\gamma}}\left\|\hat{\kappa}_{n}^{p^{\prime} \gamma}\right\|_{L^{\frac{\gamma+2}{2 \gamma}}\left(\mu_{Y}\right)}\|v w\|_{L^{1}\left(\mu_{\Delta}\right)}^{p^{\prime}}$. It follows that

$$
\ll \sum_{n \geq 1}|t|^{p^{\prime} \gamma} \mu_{Y}(\sigma>n)^{\frac{2-\gamma}{2+\gamma}}\left\|\hat{\kappa}_{n}\right\|_{L^{p^{\prime} \frac{\gamma+2}{2}\left(\mu_{Y}\right)}}^{p^{\prime} \gamma}\|v w\|_{L^{1}\left(\mu_{\Delta}\right)}^{p^{\prime}} \ll|t|^{p^{\prime} \gamma}\|v w\|_{\mathcal{B}}^{p^{\prime}}
$$

for any $\gamma \in\left(1, \frac{4}{p^{\prime}}-2\right)$, since $p^{\prime} \frac{\gamma+2}{2} \in(1,2)$ and $\left\|\hat{\kappa}_{n}\right\|_{L^{p^{\prime}} \frac{\gamma+2}{2}}\left(\mu_{Y}\right) \leq n\|\hat{\kappa}\|_{L^{p^{\prime}} \frac{\gamma+2}{2}\left(\mu_{\Delta}\right)}$ and $\mu_{Y}(\sigma>n) \ll \theta_{1}^{n}$. Second, using $\left|e^{i y}-1\right| \leq|y|$, it comes

$$
\begin{aligned}
& \left(I_{2}(t)\right)^{p^{\prime}}:=|t|^{p^{\prime}} \int_{\Delta}\left|\left(e^{i t \cdot \hat{\kappa}_{\omega(x)}}-1\right) \Pi_{0}^{\prime}(v w)\right|^{p^{\prime}} \circ \pi_{0} d \mu_{\Delta}(x) \ll|t|^{2 p^{\prime}} \sum_{n \geq 1} \int_{\sigma>n}\left|\hat{\kappa}_{n} \Pi_{0}^{\prime}(v w)\right|^{p^{\prime}} d \mu_{Y} \\
& \ll|t|^{2 p^{\prime}}\left\|1_{Y} \Pi_{0}^{\prime}(v w)\right\|_{\infty} \sum_{n \geq 1} \mu(\sigma>n)^{\frac{2-p^{\prime}}{p^{\prime}+2}}\left\|\hat{\kappa}_{n}^{p^{\prime}}\right\|_{L^{\frac{p^{\prime}+2}{2 p^{\prime}}}\left(\mu_{\Delta}\right)} \\
& \ll|t|^{2 p^{\prime}}\left\|1_{Y} \Pi_{0}^{\prime}(v w)\right\|_{\infty} \ll|t|^{2 p^{\prime}}\|(v, w)\|,
\end{aligned}
$$


proceeding as for $I_{1}$ and using $\left\|1_{Y} \Pi_{0}^{\prime}(v w)\right\|_{\infty} \ll\|(v, w)\|$. Third

$$
\begin{aligned}
\left(I_{3}(t)\right)^{p^{\prime}} & :=\int_{\Delta}\left|e^{i t \cdot \hat{\kappa}_{\omega(x)}}\left(\Pi_{t}-\Pi_{0}-t \Pi_{0}^{\prime}\right)(v w)\right|^{p^{\prime}} \circ \pi_{0}(x) d \mu_{\Delta}(x) \\
& =O\left(|t|^{\gamma p^{\prime}}\left(\|v w\|_{\mathcal{B}}+\|w\|_{\mathcal{B}_{0}}\|v\|_{L^{b}\left(\mu_{\Delta}\right)}\right)^{p^{\prime}}\right)
\end{aligned}
$$

with $\gamma$ as in Proposition 5.4. For the last term, we observe that the expansion of $\lambda$ at 0 implies in particular that $1>\left|\lambda_{t}\right|>e^{-a_{0} \frac{|t|^{2} \log (1 /|| t \mid)}{2}}$ if $t$ is small enough and so

$$
\begin{aligned}
\left|I_{4}(t)\right|^{p^{\prime}} & =\int_{\Delta}\left|\left(\lambda_{t}^{-\omega(x)}-1\right) e^{i t \cdot \hat{\kappa}_{\omega(x)}} \Pi_{t}(v w)\right|^{p^{\prime}} \circ \pi_{0}(x) d \mu_{\Delta}(x) \\
& \leq \int_{\Delta}\left(\omega(x)\left|\lambda_{t}-1\right| e^{a_{0} \frac{|t|^{2} \log (1 /|t|)}{2}(\omega(x)-1)}\left\|1_{Y} \Pi_{t}(v w)\right\|_{\infty}\right)^{p^{\prime}} d \mu_{\Delta}(x) \\
& \ll\left(|t|^{2} \log (1 /|t|)\left\|1_{Y} \Pi_{t}(v w)\right\|_{\infty}\right)^{p^{\prime}} \sum_{n \geq 1} \int_{\sigma>n} n^{p^{\prime}} e^{a_{0} p^{\prime} \frac{n|t|^{2} \log (1 /|t|)}{2}} d \mu_{Y}
\end{aligned}
$$

and so $\left|I_{4}(t)\right|^{p^{\prime}} \ll\left(|t|^{2} \log (1 /|t|)\left\|1_{Y} \Pi_{t}(v w)\right\|_{\infty}\right)^{p^{\prime}} \sum_{n \geq 1} n^{p^{\prime}} e^{a_{0} p^{\prime} \frac{n|t|^{2} \log (1 /|t|)}{2}} \mu_{Y}(\sigma>$ $n)$. Thus

$$
\left|I_{4}(t)\right|^{p^{\prime}} \ll\left(|t|^{2} \log (1 /|t|)\|(v, w)\|\right)^{p^{\prime}}
$$

for small $|t|$ since $\mu_{Y}(\sigma>n) \ll \theta_{1}^{n}$ and $\left\|1_{Y} \Pi_{t}(v w)\right\|_{\infty} \ll\|(v, w)\|$ (see (70)). So, taking $\gamma$ as in Proposition 5.3, $\left\|\left(\Pi_{t}-\Pi_{0}-t \cdot \Pi_{0}^{\prime}\right)(v w)\right\|_{L^{p^{\prime}}\left(\mu_{\Delta}\right)} \ll$ $|t|^{\gamma}\left(\|v w\|_{\mathcal{B}}+\|w\|_{\mathcal{B}_{0}}\|v\|_{L^{b}\left(\mu_{\Delta}\right)}\right)$.

\section{Expansion of $\lambda_{t}$ : Proof of Proposition 3.3}

Let $v_{t}$ be the eigenfunction of $P_{t}$ associated with $\lambda_{t}$ so that $\mu_{\Delta}\left(v_{t}\right)=1$, that is $v_{t}=$ $\frac{\Pi_{t} 1_{\Delta}}{\mu_{\Delta}\left(\Pi_{t} 1_{\Delta}\right)}$. Using a classical argument (see, for instance, [2]), we write

$$
\begin{aligned}
1-\lambda_{t} & =\int_{\Delta}\left(P_{0}-P_{t}\right) v_{t} d \mu_{\Delta}=\int_{\Delta}\left(1-e^{i t \cdot \hat{\kappa}}\right) v_{t} d \mu_{\Delta} \\
& =\int_{\Delta}\left(1-e^{i t \cdot \hat{\kappa}}\right) d \mu_{\Delta}+\int_{\Delta}\left(1-e^{i t \cdot \hat{\kappa}}\right)\left(v_{t}-v_{0}\right) d \mu_{\Delta}:=\Psi(t)+V(t) .
\end{aligned}
$$

Estimates of $V(t)$ and $\Psi(t)$ are given respectively in Lemmas 6.1 and 6.2. $\Psi(t)$ is the pure scalar part which will be estimated as if dealing with the characteristic function of an i.i.d. process and for this we only need to exploit Lemma 4.2. The function $V(t)$ will be estimated via the estimates used in the proof of Proposition 5.3. We start with the latter.

Lemma 6.1. There exists $C>0$ such that for all $t \in B_{\delta}(0),|V(t)| \leq C t^{2}$. 
Proof. Note that $v_{t}-v_{0}=\frac{\Pi_{t}\left(1_{\Delta}\right)-\mu_{\Delta}\left(\Pi_{t}\left(1_{\Delta}\right)\right)}{\mu_{\Delta}\left(\Pi_{t}\left(1_{\Delta}\right)\right)}$. Therefore it is enough to prove that

$$
\left|\int_{\Delta}\left(1-e^{i t \cdot \hat{\kappa}}\right)\left(\Pi_{t}\left(1_{\Delta}\right)-\mu_{\Delta}\left(\Pi_{t}\left(1_{\Delta}\right)\right)\right) d \mu_{\Delta}\right| \ll t^{2} .
$$

We know from Proposition (5.3) that $\mu_{\Delta}\left(\left(\Pi_{t}-\Pi_{0}\right)\left(1_{\Delta}\right)\right)=O(t)$, which implies that

$$
\left|\int_{\Delta}\left(1-e^{i t \cdot \hat{\kappa}}\right) \mu_{\Delta}\left(\left(\Pi_{t}-\Pi_{0}\right)\left(1_{\Delta}\right)\right) d \mu_{\Delta}\right| \ll t^{2}\|\hat{\kappa}\|_{L^{1}\left(\mu_{\Delta}\right)} \ll t^{2} .
$$

It remains to estimate $V_{0}(t):=\int_{\Delta}\left(1-e^{i t \cdot \hat{\kappa}}\right)\left(\Pi_{t}-\Pi_{0}\right)\left(1_{\Delta}\right) d \mu_{\Delta}$. To this end, thanks to (44), we use the following decomposition similar to (72)

$$
\begin{aligned}
\left(\Pi_{t}-\Pi_{0}\right) 1_{\Delta}(x)= & \lambda_{t}^{-\omega(x)} e^{i t \hat{\kappa}_{\omega(x)}\left(\pi_{0}(x)\right)} \Pi_{t}\left(1_{\Delta}\right) \circ \pi_{0}(x)-1 \\
= & \left(e^{i t \cdot \hat{\kappa}_{\omega(x)}\left(\pi_{0}(x)\right)}-1\right)+\left[e^{i t \cdot \hat{\kappa}_{\omega(x)}}\left(\Pi_{t}-\Pi_{0}\right)\left(1_{\Delta}\right)\right] \circ \pi_{0}(x) \\
& +\left(\lambda_{t}^{-\omega(x)}-1\right)\left[e^{i t \cdot \hat{\kappa}_{\omega(x)}} \Pi_{t}\left(1_{\Delta}\right)\right] \circ \pi_{0}(x),
\end{aligned}
$$

which leads to

$$
\begin{aligned}
\left|\int_{\Delta}\left(1-e^{i t \cdot \hat{\kappa}}\right)\left(\Pi_{t}-\Pi_{0}\right)\left(1_{\Delta}\right) d \mu_{\Delta}\right| \leq J_{1}(t)+J_{2}(t)+J_{3}(t), \\
J_{1}(t):=\left|\int_{\Delta}\left(1-e^{i t \cdot \hat{\kappa}(x)}\right)\left(e^{i t \cdot \hat{\kappa}_{\omega(x)}\left(\pi_{0}(x)\right)}-1\right) d \mu_{\Delta}(x)\right|, \\
J_{2}(t):=\int_{\Delta}\left|\left(1-e^{i t \cdot \hat{\kappa}(x)}\right) e^{i t \hat{\kappa}_{\omega(x)}}\left(\Pi_{t}-\Pi_{0}\right)\left(1_{\Delta}\right)\right| \circ \pi_{0}(x) d \mu_{\Delta}(x), \\
J_{3}(t):=\int_{\Delta}\left|\left(1-e^{i t \cdot \hat{\kappa}(x)}\right)\left(\lambda_{t}^{-\omega(x)}-1\right) e^{i t \kappa_{\omega}(x)} \Pi_{t}\left(1_{\Delta}\right)\right| \circ \pi_{0}(x) d \mu_{\Delta}(x) .
\end{aligned}
$$

First let us prove that

$$
\begin{gathered}
J_{1}(t)=O\left(t^{2}\right) \text { as } t \rightarrow 0 \\
J_{1}(t)=\left|\int_{Y} \sum_{k=0}^{\sigma-1}\left(1-e^{i t \cdot \hat{\kappa} \circ f^{k}}\right)\left(1-e^{i t \cdot \hat{\kappa}_{k}}\right) d \mu_{\Delta}\right| \leq t^{2} \int_{Y} \sum_{k=1}^{\sigma-1}\left|\hat{\kappa} \circ f^{k}\right|\left|\hat{\kappa}_{k}\right| d \mu_{\Delta} .
\end{gathered}
$$

To prove that $J_{1}(t)=O\left(t^{2}\right)$, we will use the fact that, due to [30, Propositions 11-12] and [30, Lemma 16], there exists $\varepsilon>0$ such that, for every $V$,

$\mu_{\Delta}\left(A_{n, V}\right)=O\left(n^{-3-\varepsilon}\right) \quad$ with $A_{n, V}:=\left\{\hat{\kappa}=n, \quad \exists|j| \leq V \log (n+2),\left|\hat{\kappa} \circ f^{j}\right|>n^{4 / 5}\right\}$.

For any $y \in Y$, we write $\ell(y)$ for the largest integer in $\{1, \ldots, \sigma(y)-1\}$ such that

$$
N(y):=\sup _{k=1, \ldots, \sigma(y)-1}\left|\hat{\kappa} \circ f^{k}(y)\right|=\left|\hat{\kappa} \circ f^{\ell(y)}(y)\right| .
$$


Set $Y_{n}:=Y \cap\left\{\hat{\kappa} \circ f^{\ell(y)}=n, Y_{n}^{\prime}:=\left\{y \in Y_{n}: \sigma(y)<b \log (n+2)\right\}\right.$ and also $Y_{n}^{(0)}:=$ $\left\{y \in Y_{n}^{\prime}: \forall j<\sigma(y),\left|\hat{\kappa} \circ f^{j}\right| \leq n^{4 / 5}\right\}$. Notice that

$$
\begin{aligned}
& \int_{Y} \sum_{k=1}^{\sigma-1}\left|\hat{\kappa} \circ f^{k}\right|\left|\hat{\kappa}_{k}\right| d \mu_{\Delta} \leq \sum_{n \geq 0} \int_{Y_{n}^{\prime}} \sum_{k=1}^{b \log (n+2)-1}\left|\hat{\kappa} \circ f^{k}\right|\left|\hat{\kappa}_{k}\right| d \mu_{\Delta}+\sum_{n \geq 0} \int_{Y_{n} \backslash Y_{n}^{\prime}} n^{2} \sigma d \mu_{\Delta} \\
& \leq \sum_{n \geq 0} \int_{Y_{n}^{(0)}} b n^{9 / 5} \log (n+2)+\sum_{n \geq 0} \int_{Y_{n}^{\prime} \backslash Y_{n}^{(0)}} n^{2} b \log (n+2) d \mu_{\Delta}+\sum_{n \geq 0} n^{2} \mathbb{E}_{\mu_{\Delta}}\left[\sigma 1_{Y \backslash Y_{n}^{\prime}}\right] \\
& \leq \sum_{n \geq 0} b n^{9 / 5} \log (n+2) \mu_{\Delta}\left(Y_{n}^{\prime}\right)+\sum_{n \geq 0} n^{2} b \log (n+2) \mu_{\Delta}\left(Y_{n}^{\prime} \backslash Y_{n}^{(0)}\right) \\
& \quad+\sum_{n \geq 0} n^{2} \sum_{m \geq b \log (n+2)} \mu_{\Delta}(\sigma>m) .
\end{aligned}
$$

The last term of the above right hand side is less than $\sum_{n \geq 0} n^{2} \sum_{m \geq b \log (n+2)} C_{1} \theta_{1}^{m} \leq$ $\sum_{n \geq 0} O\left(n^{2} \theta_{1}^{b \log (n+2)}\right)<\infty$ by taking $b$ large enough. The other terms are dominated

$$
\begin{aligned}
& \sum_{n \geq 0} O\left(n^{9 / 5} \log (n+2) \sum_{m=0}^{b \log (n+2)} \mu_{\Delta}(\hat{\kappa}=n)\right)+\sum_{n \geq 0} n^{2} \log (n+2) \sum_{m=0}^{b \log (n+2)} \mu_{\Delta}\left(A_{n, b}\right) \\
& \leq \sum_{n \geq 0} O\left(n^{9 / 5-3}(\log (n+2))^{2}\right)+\sum_{n \geq 0} n^{2-3-\varepsilon}(\log (n+2))^{2}<\infty,
\end{aligned}
$$

Combined with (81), this ends the proof of (80). Second, due to Proposition 5.4

$$
\begin{aligned}
J_{2}(t) & =\int_{\Delta}\left|\left(1-e^{i t \cdot \hat{\kappa}(x)}\right) e^{i t \hat{\kappa}_{\omega(x)}}\left(\Pi_{t}-\Pi_{0}\right)\left(1_{\Delta}\right)\right| \circ \pi_{0}(x) d \mu_{\Delta}(x) \\
& \ll \int_{\Delta}\left|t \cdot \kappa(x)\left(\Pi_{t}-\Pi_{0}\right)\left(1_{\Delta}\right)\right| \circ \pi_{0}(x) d \mu_{\Delta}(x) \ll t^{2}\|\hat{\kappa}\|_{L^{1}\left(\mu_{\Delta}\right)},
\end{aligned}
$$

and finally, using the fact that $1>\left|\lambda_{t}\right|>e^{-\frac{t^{2} \log (1 /|t|)}{2}}$ if $t$ is small enough,

$$
\begin{aligned}
\left|J_{3}(t)\right| & =\int_{\Delta}\left|\left(1-e^{i t \cdot \hat{\kappa}(x)}\right)\left(\lambda_{t}^{-\omega(x)}-1\right) e^{i t \kappa_{\omega}(x)} \Pi_{t}\left(1_{\Delta}\right)\right| \circ \pi_{0}(x) d \mu_{\Delta}(x) \\
& \leq \int_{\Delta}|t \cdot \hat{\kappa}(x)| \omega(x)\left|\lambda_{t}-1\right| e^{\frac{t^{2} \log (1 /|t|)}{2}(\omega(x)-1)}\left\|1_{Y} \Pi_{t}\left(1_{\Delta}\right)\right\|_{\infty} d \mu_{\Delta}(x) \\
& \ll t^{3} \log (1 /|t|) \sum_{n \geq 1} \int_{Y \cap\{\sigma>n\}}\left|\hat{\kappa} \circ f^{n}\right| n e^{\frac{n t^{2} \log (1 /|t|)}{2}} d \mu_{Y}
\end{aligned}
$$

Thus $\left|J_{3}(t)\right| \ll t^{3} \log (1 /|t|) \sum_{n \geq 1} n e^{\frac{n t^{2} \log (1 /|t|)}{2}}\|\hat{\kappa}\|_{L^{p}\left(\mu_{\Delta}\right)}\left(\mu_{Y}(\sigma>n)\right)^{1 / q}$ and it follows that

$$
\left|J_{3}(t)\right| \ll t^{3} \log (1 /|t|) \sum_{n \geq 1} n e^{\frac{n t^{2} \log (1 /|t|)}{2}}\|\hat{\kappa}\|_{L^{p}\left(\mu_{\Delta}\right)}\left(C_{1} \theta_{1}^{n}\right)^{1 / q}=O\left(t^{2}\right),
$$

by taking $p<2$ close to 2 and using the fact that for $|t|$ small enough $e^{\frac{t^{2} \log (1 /|t|)}{2}} \theta_{1}<1$. Combining (77), (79), (80), (82) and (83), we obtain (76) and conclude. 
To complete the proof of Proposition 3.3, we still need to estimate $\Psi(t)$ in (75). The next lemma can be viewed as an extension of the asymptotic of the pure scalar quantity in [1, Proof of Theorem 3.1] under the conclusion of Lemma 4.2 via the arguments used in $[19,31]$.

Lemma 6.2. Let $\hat{\kappa}_{0}: \Delta \rightarrow \mathbb{Z}^{d}$ be a $\mu_{\Delta}$-centered function. Set $\Psi(t):=\int_{\Delta}(1-$ $\left.e^{i t \cdot \hat{\kappa}_{0}}\right) d \mu_{\Delta}$. Assume that there exists $M_{0}>0$ such that $\left\{\left|\hat{\kappa}_{0}\right| \geq M_{0}\right\}=\bigcup_{(L, w) \in \mathcal{S}}\left\{\hat{\kappa}_{0} \in\right.$ $L+\mathbb{N} w\}$ with $\mathcal{S}$ a finite subset of $\left(\mathbb{Z}^{2}\right)^{2}$ such that the lattices $L+\mathbb{N} w \subset \mathbb{Z}^{d}$ are disjoint for distinct $(L, w) \in \mathcal{S}$ outside the open ball $B\left(0, M_{0}\right)$. Assume moreover that

$$
\forall(L, w) \in \mathcal{S}, \quad \mu_{\Delta}\left(\hat{\kappa}_{0}=L+n w\right)=\frac{c_{L, w}}{n^{3}}+O\left(n^{-4}\right), \quad \text { as } n \rightarrow+\infty .
$$

Then, as $t \rightarrow 0, \Psi(t)=\left.\sum_{(L, w) \in \mathcal{S}} \frac{c_{L, w}}{2}(t \cdot w)^{2} \log || t\right|^{-1} \mid+O\left(t^{2}\right)$.

Proof. We start by writing $\Psi(t)=\int_{\Delta}\left(1-e^{i t \cdot \hat{\kappa}_{0}}\right) d \mu_{\Delta}=\int_{\Delta}\left(1+i t \cdot \hat{\kappa}_{0}-e^{i t \cdot \hat{\kappa}_{0}}\right) d \mu_{\Delta}$. So

$$
\begin{aligned}
\Psi(t) & =\sum_{(L, w) \in \mathcal{S}} \sum_{n=1}^{\left\lfloor|t|^{-1}\right\rfloor}\left(1+i t \cdot(L+n w)-e^{i t \cdot(L+n w)}\right) \mu_{\Delta}\left(\hat{\kappa}_{0}=L+n w\right)+O\left(t^{2}\right) \\
& =\sum_{(L, w) \in \mathcal{S}} \sum_{n=1}^{\left\lfloor|t|^{-1}\right\rfloor}\left(1+i t \cdot(L+n w)-e^{i t \cdot(L+n w)}\right)\left(\frac{c_{L, w}}{n^{3}}+O\left(n^{-4}\right)\right)+O\left(t^{2}\right) \\
& =\sum_{(L, w) \in \mathcal{S}} \sum_{n=1}^{\left\lfloor|t|^{-1}\right\rfloor}\left(1+i t \cdot(L+n w)-e^{i t \cdot(L+n w)}\right) \frac{c_{L, w}}{n^{3}}+O\left(t^{2}\right),
\end{aligned}
$$

using the properties of $M_{0}, \mathcal{S}$. Setting $a_{L, w, n}:=\frac{c_{L, w}}{n^{3}}, A_{L, w, n}:=\sum_{k \geq n} a_{L, w, k}$ and $b_{L, w, n}:=1+i t \cdot(L+n w)-e^{i t \cdot(L+n w)}$, using the Abel transform, we obtain

$$
\begin{aligned}
\Psi(t) & =O\left(t^{2}\right)+\sum_{(L, w) \in \mathcal{S}} \sum_{n=1}^{\left\lfloor|t|^{-1}\right\rfloor} a_{L, w, n} b_{L, w, n} \\
& =O\left(t^{2}\right)+\sum_{(L, w) \in \mathcal{S}}\left\{A_{L, w, 1} b_{L, w, 1}-A_{L, w,\left\lfloor|t|^{-1}\right\rfloor+1} b_{L, w,\left\lfloor|t|^{-1}\right\rfloor}+\sum_{n=2}^{\left\lfloor|t|^{-1}\right\rfloor} A_{L, w, n}\left(b_{L, w, n}-b_{L, w, n-1}\right)\right\} \\
& =O\left(t^{2}\right)+\sum_{n=2}^{\left\lfloor|t|^{-1}\right\rfloor} \sum_{(L, w) \in \mathcal{S}}\left\{\left(\frac{c_{L, w}}{2 n^{2}}+O\left(n^{-3}\right)\right)\left(i t \cdot w+e^{i t \cdot(L+(n-1) w)}-e^{i t \cdot(L+n w)}\right)\right\}
\end{aligned}
$$

and thus

$$
\begin{aligned}
\Psi(t) & =O\left(t^{2}\right)+\sum_{n=2}^{\left\lfloor|t|^{-1}\right\rfloor} \sum_{(L, w) \in \mathcal{S}}\left\{\left(\frac{c_{L, w}}{2 n^{2}}+O\left(n^{-3}\right)\right)\left(i t \cdot w\left(1-e^{i t \cdot(L+n w)}\right)+O\left(t^{2}\right)\right)\right\} \\
& =O\left(t^{2}\right)+\sum_{n=2}^{\left\lfloor|t|^{-1}\right\rfloor} \sum_{(L, w) \in \mathcal{S}}\left\{\left(\frac{c_{L, w}}{2 n^{2}}+O\left(n^{-3}\right)\right)(i t \cdot w)\left(-i t \cdot(L+n w)+O\left(t^{2} n^{2}\right)\right)\right\}
\end{aligned}
$$




$$
=O\left(t^{2}\right)+(t \cdot w)^{2} \sum_{(L, w) \in \mathcal{S}} \sum_{n=2}^{\left\lfloor|t|^{-1}\right\rfloor} \frac{c_{L, w}}{2 n}=O\left(t^{2}\right)+\left.\sum_{(L, w) \in \mathcal{S}} \frac{c_{L, w}}{2}(t \cdot w)^{2} \log || t\right|^{-1} \mid .
$$

Proof of Proposition 3.3. We first observe that Lemma 4.2 ensures that the general conditions of Lemma 6.2 are satisfied with $c_{L, w}:=\sum_{(A, B) \in E_{L, w}} \frac{d_{A}^{2} \mathfrak{a}_{(A, B)}}{2|\partial \bar{Q}||w|}$, and with $\mathcal{S}$ is the set of $(w, L) \in\left(\mathbb{Z}^{2}\right)^{2}$, with $w$ with co-prime coordinates and $L \in \mathcal{E}_{w}$ (where $\mathcal{E}_{w}$ is the set of $L \in \mathbb{Z}^{2}$ such that $\left.L \cdot w \geq 0>(L-w) \cdot w\right)$ for which there exist $(A, B) \in E_{L, w}$. The finiteness of $\mathcal{S}$ comes then from the finiteness of $\mathcal{A}$ and the finiteness of the possibilities for $B$ once $(A, w)$ is fixed, the finiteness of $L$ once $A, w, B$ are fixed comes from our constraint on $L$. The disjointness assumption of Lemma 6.2 comes from our first conditions on $w$ and $L$. Since $1-\lambda_{t}=\Psi(t)+V(t)$, due to Lemmas 6.1 and 6.2, we know that

$$
1-\lambda_{t}=\left.\sum_{(L, w) \in \mathcal{S}} \frac{c_{L, w}}{2}(t \cdot w)^{2} \log || t\right|^{-1} \mid+O\left(t^{2}\right) .
$$

For any prime $w \in \mathbb{Z}^{2}$, due to Lemma 4.2 and Corollary 4.3 ,

$$
\sum_{L:(L, w) \in \mathcal{S}} c_{L, w}=\sum_{C \in \mathcal{C}: w_{C}= \pm w} \frac{\mathfrak{d}_{C}^{2}}{|\partial \bar{Q}|\left|w_{C}\right|} .
$$

Therefore $1-\lambda_{t}=\left.2 \sum_{C \in \mathcal{C}} \frac{\mathfrak{d}_{C}^{2}}{2|\partial \bar{Q}|\left|w_{C}\right|}\left(t \cdot w_{C}\right)^{2} \log || t\right|^{-1} \mid+O\left(t^{2}\right)$.

\section{Expansions in the Local Limit Theorem and of Decorrelation Rate}

7.1. Expansion in the local limit theorem in a general context. Let $\left(\Delta, f, \mu_{\Delta}\right)$ be a probability preserving dynamical system with transfer operator $P$. Let $p, p_{0}, p_{1} \in$ $[1,+\infty]$ with $p_{0} \leq p_{1}$. Set $\hat{\kappa}_{n}:=\sum_{k=0}^{n-1} \hat{\kappa} \circ f^{k}$, with $\hat{\kappa}: \Delta \rightarrow \mathbb{Z}^{d}$ integrable with zero mean. Assume that, for every $t \in[-\pi, \pi]^{d}$, the operator $P_{t}: f \mapsto P\left(e^{i t \cdot \hat{\kappa}} f\right)$ acts on a complex Banach space $\mathcal{B}$ of functions $f: \Delta \rightarrow \mathbb{C}$ and satisfies the following properties. Assume that $\hat{\kappa} \in L^{p}\left(\mu_{\Delta}\right)$, that $\mathcal{B} \hookrightarrow L^{p_{1}}\left(\mu_{\Delta}\right)$ and that there exists $\beta \in(0, \pi)$ such that for every $t \in[-\beta, \beta]^{d}$,

$$
P_{t}^{n}=\lambda_{t}^{n} \Pi_{t}+N_{t}^{n},
$$

Assume moreover that there exists an invertible positive symmetric matrix $A$ such that

$$
\lambda_{t}=1-A t \cdot t \log (1 /|t|)+O\left(t^{2}\right) \text {, i.e. } \lambda_{t}=e^{-A t \cdot t \log (1 /|t|)+O\left(t^{2}\right)} .
$$

Moreover assume either that there exists $\gamma \in(0,1]$ such that

$$
\Pi_{t}:=\mathbb{E}_{\mu_{\Delta}}[\cdot] \mathbf{1}_{\Delta}+O\left(|t|^{\gamma}\right) \text { in } \mathcal{L}\left(\mathcal{B}, L^{p_{0}}\left(\mu_{\Delta}\right)\right)
$$


or that there exists $\gamma^{\prime} \in(0,1]$ such that

$$
\exists \Pi_{0}^{\prime} \in \mathcal{L}\left(\mathcal{B}, L^{p_{0}}\left(\mu_{\Delta}\right)\right), \Pi_{t}:=\mathbb{E}_{\mu_{\Delta}}[\cdot] \mathbf{1}_{\Delta}+t \cdot \Pi_{0}^{\prime}+O\left(t^{1+\gamma^{\prime}}\right) \text { in } \mathcal{L}\left(\mathcal{B}, L^{p_{0}}\left(\mu_{\Delta}\right)\right) .
$$

Note that, due to Proposition 3.1, under (12), both (87) and (88) are satisfied for the tower $\left(\Delta, f, \mu_{\Delta}\right)$ associated to the discrete infinite horizon Lorentz gas. Let $q_{0}, q_{1} \in[1,+\infty]$ be such that $\frac{1}{q_{i}}+\frac{1}{p_{i}}=1$. Let us define

$$
\begin{aligned}
& I_{0}(X):=\frac{e^{-\frac{1}{2} A^{-1} X \cdot X}}{\sqrt{(2 \pi)^{d} \operatorname{det} A}}, \quad I_{2}(X):=-\frac{A^{-1} X \cdot X-d}{\sqrt{(2 \pi)^{d} \operatorname{det} A}} e^{-\frac{1}{2} A^{-1} X \cdot X}, \\
& I_{1}(X):=-i \frac{A^{-1} X}{\sqrt{(2 \pi)^{d} \operatorname{det}(A)}} e^{-\frac{1}{2} A^{-1} X \cdot X}, \quad I_{3}(X):=-i \frac{A^{-1} X\left(d+2-A^{-1} X \cdot X\right)}{\sqrt{(2 \pi)^{d} \operatorname{det}(A)}} e^{-\frac{1}{2} A^{-1} X \cdot X} .
\end{aligned}
$$

Under these assumptions, we state the following general local limit theorem with expansion, that can be read first considering $k=0$. The generalization to $k=O(n / \log n)$ will be useful in the proof of our main results (Theorems 2.2 and 2.4) due to approximations of observables $\phi, \psi$ using functions that are constant on every stable curve. We set $a_{n}:=\sqrt{n \log n}$.

Proposition 7.1. Assume (84)-(86).

If (87) holds, then, for every $h \in \mathcal{B}$, for $g \in L^{q_{0}}\left(\mu_{\Delta}\right)$, uniformly in $N \in \mathbb{Z}^{d}$

$$
\begin{aligned}
& \mathbb{E}_{\mu_{\Delta}}\left[h \mathbf{1}_{\left\{\hat{\kappa}_{n} \circ f^{k}=N\right\}} g \circ f^{n}\right]=\frac{\mathbb{E}_{\mu_{\Delta}}[h] \mathbb{E}_{\mu_{\Delta}}[g]}{a_{n}^{d}}\left(I_{0}\left(\frac{N}{a_{n}}\right)-\frac{I_{2}\left(\frac{N}{a_{n}}\right) \log \log n+O(1)}{2 \log n}\right) \\
& +O\left(a_{n}^{-d}\left(a_{n}^{-\gamma}+\left(k / a_{n}\right)^{\min \left(1, \frac{p}{q_{1}}, \frac{p}{p_{0}}\right)}\right)\|g\|_{L^{q_{0}\left(\mu_{\Delta}\right)}}\left(\sup _{t}\left\|P_{t}^{k} P^{k} h\right\|_{\mathcal{B}}\right),\right.
\end{aligned}
$$

for $k=0$ (without additional assumption on $p_{1}$ ) and uniformly in $k \leq C n / \log n$. If (88) holds, then, for every $h \in \mathcal{B}$, for $g \in L^{q_{0}}\left(\mu_{\Delta}\right)$, uniformly in $N \in \mathbb{Z}^{d}$,

$$
\begin{aligned}
\mathbb{E}_{\mu_{\Delta}} & {\left[h \mathbf{1}_{\left\{\hat{\kappa}_{n} \circ f^{k}=N\right\}} g \circ f^{n}\right] } \\
= & \frac{1}{a_{n}^{d}} \mathbb{E}_{\mu_{\Delta}}[h] \mathbb{E}_{\mu_{\Delta}}[g]\left(I_{0}\left(\frac{N}{a_{n}}\right)-\frac{I_{2}\left(\frac{N}{a_{n}}\right) \log \log n+O(1)}{2 \log n}\right) \\
& +\frac{C_{k}(g, h)}{a_{n}^{d+1}}\left(I_{1}\left(\frac{N}{a_{n}}\right)-\frac{I_{3}\left(\frac{N}{a_{n}}\right)}{2} \frac{\log \log n}{\log n}\right)+O\left(\frac{\|g\|_{L^{q_{0}}\left(\mu_{\Delta}\right)} \sup _{u}\left\|P_{u}^{k} P^{k} h\right\|_{\mathcal{B}}}{a_{n}^{d+1} \log n}\right) \\
& +O\left(\frac{1}{(2 \pi)^{d}\left(a_{n}\right)^{d+1}} \int_{\left[-\beta a_{n}, \beta a_{n}\right]^{d}} \frac{\left.|u| e^{-\frac{c_{0} \min \left(|u|^{2-\epsilon},|u|^{2+\epsilon}\right)}{2}}\left|\mathbb{E}_{\mu_{\Delta}}\left[g \Pi_{0}^{\prime}\left(P_{u / a_{n}}^{k} P^{k} h-P^{2 k} h\right)\right]\right| d u\right),}{}\right.
\end{aligned}
$$

with $C_{k}(g, h):=\mathbb{E}_{\mu_{\Delta}}\left[g \Pi_{0}^{\prime} P^{2 k} h\right]+i \mathbb{E}_{\mu_{\Delta}}\left[\hat{\kappa}_{k} g\right] \mathbb{E}_{\mu_{\Delta}}[h]+i \mathbb{E}_{\mu_{\Delta}}[g] \mathbb{E}_{\mu_{\Delta}}\left[\hat{\kappa}_{k} P^{k} h\right]$, for $k=0$ (without additional assumption on $p_{1}, p_{0}, q_{0}$ ) and uniformly in $k \leq$ $C a_{n}^{\frac{\tilde{\gamma}}{1+\tilde{\gamma}}} /(\log n)^{1 /(1+\tilde{\gamma})}$ with $\tilde{\gamma}=\min \left(1, \frac{p\left(q_{0}-1\right)-q_{0}}{q_{0}}, \frac{p-q_{1}}{q_{1}}\right)$ if $q_{1}<p, q_{0}<+\infty$, $\frac{1}{q_{0}}+\frac{1}{p}<1$. 
When $k=0$, one can take $p_{0}=p_{1}=1, q_{0}=q_{1}=\infty$ and that the last error term in the second estimate of Proposition 7.1 vanishes.

Remark 7.2. If $k=0$ and $\mathbb{E}_{\mu_{\Delta}}[g] \mathbb{E}_{\mu_{\Delta}}[h]=0$, the second estimate of Proposition 7.1 provides an estimate in $O\left(a_{n}^{-d-1} / \log n\right)$ if $N$ is fixed or uniformly bounded, but provides an estimate with a leading term (non null in general) of order $a_{n}^{-d-1}$ if $N$ has order $a_{n}$.

Proof of Proposition 7.1. We assume that $\beta<1$, that

$$
\forall t \in[-\beta, \beta]^{d}, \quad e^{-2 A t \cdot t \log (1 /|t|)} \leq\left|\lambda_{t}\right| \leq e^{-\frac{1}{2} A t \cdot t \log (1 /|t|)}
$$

for $|\cdot|$ the supremum norm on $\mathbb{R}^{d}$ and that $\forall y>x>\beta^{-1}, \frac{1}{2}(x / y)^{\epsilon} \leq \log (x) / \log (y) \leq$ $2(y / x)^{\varepsilon}$ (using for example Karamata's representation of slowly varying functions). This last condition will imply that, for every $n$ large enough (so that $a_{n}>\beta^{-1}$ ) and for every $|u| \in\left[-\beta a_{n}, \beta a_{n}\right]$, the following inequalities hold true

$$
\frac{1}{2} \min \left(|u|^{\varepsilon},|u|^{-\varepsilon}\right) \leq \log \left(a_{n} /|u|\right) / \log a_{n} \leq 2 \max \left(|u|^{\varepsilon},|u|^{-\varepsilon}\right) .
$$

Let $h, g$ be as in the statement of Proposition 7.1. Note that $P_{t}^{n}\left(H . G \circ f^{n}\right)=G P_{t}^{n}(H)$ (since $\left.\int_{\Delta} h P(H \cdot G \circ f) d \mu_{\Delta}=\int_{\Delta}(h \circ f) H(G \circ f) d \mu_{\Delta}=\int_{\Delta} h G P(H) d \mu_{\Delta}\right)$. Thus,

$$
\begin{aligned}
\mathbb{E}_{\mu_{\Delta}} & {\left[h \mathbf{1}_{\left\{\hat{\kappa}_{n} \circ f^{k}=N\right\}} g \circ f^{n}\right]=\frac{1}{(2 \pi)^{d}} \int_{[-\pi, \pi]^{d}} e^{-i t \cdot N} \mathbb{E}_{\mu_{\Delta}}\left[h e^{i t \cdot \hat{\kappa}_{n} \circ f^{k}} g \circ f^{n}\right] d t } \\
= & \frac{1}{(2 \pi)^{d}} \int_{[-\pi, \pi]^{d}} e^{-i t \cdot N} \mathbb{E}_{\mu_{\Delta}}\left[P^{n+k}\left(h e^{i t \cdot \hat{\kappa}_{n} \circ f^{k}} g \circ f^{n}\right)\right] d t \\
= & \frac{1}{(2 \pi)^{d}} \int_{[-\pi, \pi]^{d}} e^{-i t \cdot N} \mathbb{E}_{\mu_{\Delta}}\left[P^{k}\left(P^{n}\left(h e^{i t \cdot \hat{\kappa}_{n-k} \circ f^{k}}\left(g e^{i t \cdot \hat{\kappa}_{k}}\right) \circ f^{n}\right)\right)\right] d t
\end{aligned}
$$

Thus $\mathbb{E}_{\mu_{\Delta}}\left(h \mathbf{1}_{\left\{\hat{\kappa}_{n} \circ f^{k}=N\right\}} g \circ f^{n}\right)=\frac{1}{(2 \pi)^{d}} \int_{[-\pi, \pi]^{d}} e^{-i t \cdot N} \mathbb{E}_{\mu_{\Delta}}\left[P_{t}^{k}\left(g P^{n}\left(h e^{i t \cdot \hat{\kappa}_{n-k} \circ f^{k}}\right)\right)\right] d t$ and so

$$
\mathbb{E}_{\mu_{\Delta}}\left[h \mathbf{1}_{\left\{\hat{\kappa}_{n} \circ f^{k}=N\right\}} g \circ f^{n}\right]=\frac{1}{(2 \pi)^{d}} \int_{[-\pi, \pi]^{d}} e^{-i t \cdot N} \mathbb{E}_{\mu_{\Delta}}\left[P_{t}^{k}\left(g P_{t}^{n-2 k} P_{t}^{k} P^{k} h\right)\right] d t .
$$

Now, due to (84) and (85), $\mathbb{E}_{\mu_{\Delta}}\left(h \mathbf{1}_{\left\{\hat{\kappa}_{n} \circ f^{k}=N\right\}} g \circ f^{n}\right)$ is equal to

$$
\begin{aligned}
& \frac{1}{(2 \pi)^{d}} \int_{[-\beta, \beta]^{d}} e^{-i t \cdot N} \lambda_{t}^{n-2 k} \mathbb{E}_{\mu_{\Delta}}\left[e^{i t \cdot \hat{\kappa}_{k}} g \Pi_{t} P_{t}^{k} P^{k} h\right] d t+O\left(\theta^{n-2 k}\|g\|_{L} q_{0}\left(\mu_{\Delta}\right) \sup _{t}\left\|P_{t}^{k} P^{k} h\right\|_{\mathcal{B}}\right) \\
& \quad=\frac{1}{\left(2 \pi a_{n}\right)^{d}} \int_{\left[-\beta a_{n}, \beta a_{n}\right]^{d}} e^{-i \frac{u}{a_{n}} \cdot N} \lambda_{u / a_{n}}^{n-2 k} \mathbb{E}_{\mu_{\Delta}}\left[e^{i \frac{u}{a_{n}} \cdot \hat{\kappa}_{k}} g \Pi_{u / a_{n}} P_{u / a_{n}}^{k} P^{k} h\right] d u \\
& \quad+O\left(\theta^{n-2 k}\|g\|_{L^{q_{0}}\left(\mu_{\Delta}\right)} \sup _{t}\left\|P_{t}^{k} P^{k} h\right\|_{\mathcal{B}}\right) .
\end{aligned}
$$

Observe that $a_{n}^{2} \sim 2 n \log \left(a_{n}\right)$. This, combined with (92) and (93), ensures that there exists $c_{0}, c_{0}^{\prime}>0$ such that, for every $n$ large enough and for every $u \in\left[-\beta a_{n}, \beta a_{n}\right]^{d}$, $e^{-c_{0}^{\prime} \max \left(|u|^{2-\epsilon},|u|^{2+\epsilon}\right)} \leq e^{-\frac{2 n}{a_{n}^{2}} A u \cdot u \log \left(a_{n} /|u|\right)} \leq\left|\lambda_{u / a_{n}}^{n}\right| \leq e^{-\frac{n}{2 a_{n}^{2}} A u \cdot u \log \left(a_{n} /|u|\right)} \leq e^{-c_{0} \min \left(|u|^{2-\epsilon},|u|^{2+\epsilon}\right)}$. 
Therefore, using (87), we obtain that

$$
\left\|\lambda_{u / a_{n}}^{n-2 k}\left(\Pi_{u / a_{n}}-\mathbb{E}_{\mu_{\Delta}}[\cdot] \mathbf{1}_{\Delta}\right)\right\|_{\mathcal{L}\left(\mathcal{B}, L^{p_{0}}\left(\mu_{\Delta}\right)\right)} \leq K_{1} e^{-c_{0} \min \left(|u|^{2+\epsilon},|u|^{2-\epsilon}\right)}\left(u / a_{n}\right)^{\gamma},
$$

and so

$$
\begin{aligned}
\mathbb{E}_{\mu_{\Delta}} & \left(h \mathbf{1}_{\left\{\hat{\kappa}_{n} \circ f^{k}=N\right\}} g \circ f^{n}\right)=O\left(a_{n}^{-d-\gamma}\|g\|_{L^{q_{0}}\left(\mu_{\Delta}\right)} \sup _{t}\left\|P_{t}^{k} P^{k} h\right\|_{\mathcal{B}}\right) \\
& +\frac{1}{\left(2 \pi a_{n}\right)^{d}} \int_{\left[-\beta a_{n}, \beta a_{n}\right]^{d}} \mathbb{E}_{\mu_{\Delta}}\left[e^{i \frac{u}{a_{n}} \cdot \hat{\kappa}_{k}} g\right] \mathbb{E}_{\mu_{\Delta}}\left[e^{i \frac{u}{a_{n}} \cdot \hat{\kappa}_{k} \circ f^{k}} h\right] e^{-i \frac{u}{a_{n}} \cdot N} \lambda_{u / a_{n}}^{n-2 k} d u \\
= & \frac{1}{\left(2 \pi a_{n}\right)^{d}} \mathbb{E}_{\mu_{\Delta}}[g] \mathbb{E}_{\mu_{\Delta}}[h] \int_{\left[-\beta a_{n}, \beta a_{n}\right]^{d}} e^{-i \frac{u}{a_{n}} \cdot N} \lambda_{u / a_{n}}^{n-2 k} d u \\
& +O\left(a_{n}^{-d}\left(a_{n}^{-\gamma}+\left(k / a_{n}\right)^{\min \left(1, \frac{p}{q_{1}}\right)}+\left(k / a_{n}\right)^{\min \left(1, \frac{p}{p_{0}}\right)}\right)\|g\|_{\mathbb{L}^{q_{0}}\left(\mu_{\Delta}\right)} \sup _{t}\left\|P_{t}^{k} P^{k} h\right\|_{\mathcal{B}}\right),
\end{aligned}
$$

due to (95) combined with the two following estimates

$$
\begin{aligned}
\mathbb{E}_{\mu_{\Delta}}\left[\left(e^{i \frac{u}{a_{n}} \cdot \hat{\kappa}_{k}}-1\right) g\right] & =O\left(|u|^{\gamma_{0}} \frac{k^{\gamma_{0}}\|\hat{\kappa}\|_{L^{p}\left(\mu_{\Delta}\right)}^{\gamma_{0}}\|g\|_{\mathbb{L}^{q_{0}}\left(\mu_{\Delta}\right)}}{\left(a_{n}\right)^{\gamma_{0}}}\right) \\
\mathbb{E}_{\mu_{\Delta}}\left[\left(e^{i \frac{u}{a_{n}} \cdot \hat{\kappa}_{k} \circ f^{k}}-1\right) P^{k} h\right] & =O\left(|u|^{\gamma_{0}} \frac{k^{\gamma_{0}}\|\hat{\kappa}\|_{L^{p}\left(\mu_{\Delta}\right)}^{\gamma_{0}}\|h\|_{\mathbb{L}^{p_{1}}\left(\mu_{\Delta}\right)}}{\left(a_{n}\right)^{\gamma_{0}}}\right),
\end{aligned}
$$

with $\gamma_{0}:=\min \left(1, \frac{p}{p_{0}}, \frac{p}{q_{1}}\right)$. Here we have used Hölder inequality, the fact that $\left|e^{i x}-1\right| \leq$ $2|x|^{\gamma_{0}}$ and the following inequality applied with $s=\gamma_{0}$ and $r \in\left\{p_{0}, q_{1}\right\}$

$$
\forall r \geq 1, \quad \forall s \geq 1 / r, \quad\left\|\left|\hat{\kappa}_{k}\right|^{s}\right\|_{L^{r}\left(\mu_{\Delta}\right)}=\left\|\hat{\kappa}_{k}\right\|_{L^{r s}\left(\mu_{\Delta}\right)}^{S} \leq k^{s}\|\hat{\kappa}\|_{L^{r s}\left(\mu_{\Delta}\right)}^{s},
$$

where we used the triangular inequality for $\|\cdot\|_{L^{r s}\left(\mu_{\Delta}\right)}$ and the fact that $\left\|\hat{\kappa} \circ f^{m}\right\|_{L^{r s}\left(\mu_{\Delta}\right)}=$ $\|\hat{\kappa}\|_{L^{r s}\left(\mu_{\Delta}\right)}$ by $f$-invariance of $\mu_{\Delta}$. Moreover, due to (86) and (95) and since $k=$ $O(n / \log n)$,

$$
\begin{aligned}
\lambda_{u / a_{n}}^{n-2 k}= & \lambda_{u / a_{n}}^{n} \lambda_{u / a_{n}}^{-2 k}=e^{-\frac{n}{a_{n}^{2}} A u \cdot u\left(\log \left(a_{n} /|u|\right)\right)+O\left(n|u|^{2} / a_{n}^{2}\right)} e^{O\left(\frac{k}{n} \max \left(|u|^{2-\epsilon},|u|^{2+\epsilon}\right)\right)} \\
= & e^{-\frac{1}{2 \log n} A u \cdot u\left(\log n+\log \left(\log n /|u|^{2}\right)\right)+O\left(\max \left(|u|^{2-\epsilon},|u|^{2+\epsilon}\right) / \log n\right)} \\
= & e^{-\frac{1}{2} A u \cdot u\left(1+\frac{\log \left(\log n /|u|^{2}\right)}{\log n}\right)} \\
& +O\left(e^{-\frac{c_{0} \min \left(|u|^{2-\epsilon},|u|^{2+\epsilon}\right)}{2}} \frac{\max \left(|u|^{2-\varepsilon},|u|^{2+\varepsilon}\right)}{\log n}\right)
\end{aligned}
$$

Therefore

$$
\begin{aligned}
& \int_{\left[-\beta a_{n}, \beta a_{n}\right]^{d}} u^{\ell} e^{-i \frac{u}{a_{n}} \cdot N} \lambda_{u / a_{n}}^{n-2 k} d u=\int_{\mathbb{R}^{d}} u^{\ell} e^{-i \frac{u}{a_{n}} \cdot N} e^{-\frac{1}{2} A u \cdot u\left(1+\frac{\log \left(\log n /|u|^{2}\right)}{\log n}\right)} d u+O\left(\frac{1}{\log n}\right) \\
& \quad=\int_{\mathbb{R}^{d}} u^{\ell} e^{-i \frac{u}{a_{n}} \cdot N} e^{-\frac{1}{2} A u \cdot u}\left(1-\frac{1}{2} A u \cdot u \frac{\log \left(\log n /|u|^{2}\right)}{\log n}\right.
\end{aligned}
$$




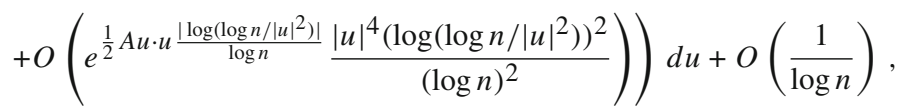

where we used $e^{x}=1+x+O\left(e^{|x|} x^{2}\right)$. Thus

$$
\begin{aligned}
& \int_{\left[-\beta a_{n}, \beta a_{n}\right]^{d}} u^{\ell} e^{-i \frac{u}{a_{n}} \cdot N} \lambda_{u / a_{n}}^{n-2 k} d u \\
& =\int_{\mathbb{R}^{d}} u^{\ell} e^{-i \frac{u}{a_{n}} \cdot N} e^{-\frac{1}{2} A u \cdot u}\left(1-\frac{1}{2} A u \cdot u \frac{\log \left(\log n /|u|^{2}\right)}{\log n}\right) \\
& +O\left(|u|^{4+\ell} e^{-\frac{1}{2} A u \cdot u\left(1-\frac{\log \log n}{\log n}-2 \frac{|\log | u||}{\log n}\right)} \frac{(\log \log n)^{2}+|\log | u||^{2}}{(\log n)^{2}}\right) d u+O\left(\frac{1}{\log n}\right), \\
& \int_{\left[-\beta a_{n}, \beta a_{n}\right]^{d}} u^{\ell} e^{-i \frac{u}{a_{n}} \cdot N} \lambda_{u / a_{n}}^{n-2 k} d u \\
& =\int_{\mathbb{R}^{d}} u^{\ell} e^{-i \frac{u}{a_{n}} \cdot N} e^{-\frac{1}{2} A u \cdot u}\left(1-\frac{1}{2} A u \cdot u \frac{\log \left(\log n /|u|^{2}\right)}{\log n}\right) \\
& +O\left(|u|^{4+\ell} e^{-\frac{1}{2} A u \cdot u\left(\frac{1}{2}-2 \max \left(|u|,|u|^{-1}\right)\right)} \frac{(\log \log n)^{2}+|\log | u||^{2}}{(\log n)^{2}}\right) d u+O\left(\frac{1}{\log n}\right) \\
& =\int_{\mathbb{R}^{d}} u^{\ell} e^{-i \frac{u}{a_{n}} \cdot N} e^{-\frac{1}{2} A u \cdot u}\left(1-\frac{1}{2} A u \cdot u \frac{\log \log n}{\log n}\right) d u+O\left(\frac{1}{\log n}\right) .
\end{aligned}
$$

Applying this formula with $\ell=0$ and using $\left(k / a_{n}\right)^{\min \left(1 / p_{1}, 1 / q_{1}\right)}=O\left((\log n)^{-1}\right)$, (96) becomes (91) with $I_{2 k}(x):=\frac{1}{(2 \pi)^{d}} \int_{\mathbb{R}^{d}} e^{-i u \cdot x}(A u \cdot u)^{k} e^{-\frac{1}{2} A u \cdot u} d u$, which coincide with (89). Indeed, with the change of variable $v=A^{1 / 2} u, I_{0}(x)=\frac{\Phi\left(A^{-1 / 2} x\right)}{\sqrt{\operatorname{det} A}}$ and $I_{2}(X)=-\frac{(\Delta \Phi)\left(A^{-1 / 2} x\right)}{\sqrt{\operatorname{det} A}}$ where $\Delta$ is the Laplacian operator and with $\Phi(x):=$ $\frac{1}{(2 \pi)^{d}} \int_{\mathbb{R}^{d}} e^{-i u \cdot x} e^{-\frac{1}{2} u \cdot u} d u=\frac{e^{-\frac{1}{2} x \cdot x}}{\sqrt{(2 \pi)^{d}}}$ the standard $d$-dimensional Gaussian density. This ends the proof of the first assertion of Proposition 7.1.

Assume from now on (88). Then,

$$
\begin{aligned}
\mathbb{E}_{\mu_{\Delta}}\left[P_{t}^{k}\left(g \Pi_{t} P_{t}^{k} P^{k} h\right)\right]= & \mathbb{E}_{\mu_{\Delta}}\left[e^{i t \cdot \hat{\kappa}_{k}} g\right] \mathbb{E}_{\mu_{\Delta}}\left[P_{t}^{k} P^{k} h\right]+t \cdot \mathbb{E}_{\mu_{\Delta}}\left[e^{i t \cdot \hat{\kappa}_{k}} g \Pi_{0}^{\prime} P_{t}^{k} P^{k} h\right] \\
& +O\left(t^{1+\gamma^{\prime}}\|g\|_{L^{q_{0}}\left(\mu_{\Delta}\right)}\left\|P_{t}^{k} P^{k} h\right\|_{\mathcal{B}}\right),
\end{aligned}
$$

and so (94) leads to

$$
\begin{aligned}
& \mathbb{E}_{\mu_{\Delta}}\left[h \mathbf{1}_{\left\{\hat{\kappa}_{n} \circ f^{k}=N\right\}} g \circ f^{n}\right]=\frac{1}{\left(2 \pi a_{n}\right)^{d}} \int_{\left[-\beta a_{n}, \beta a_{n}\right]^{d}} e^{-i \frac{u}{a_{n}} \cdot N} \lambda_{u / a_{n}}^{n-2 k} \\
& \quad\left(\mathbb{E}_{\mu_{\Delta}}\left[e^{i \frac{u}{a_{n} \cdot \hat{\kappa}_{k}}} g\right] \mathbb{E}_{\mu_{\Delta}}\left[e^{i \frac{u}{a_{n}} \cdot \hat{\kappa}_{k} \circ f^{k}} h\right]+\frac{u}{a_{n}} \cdot \mathbb{E}_{\mu_{\Delta}}\left[e^{i \frac{u}{a_{n}} \cdot \hat{\kappa}_{k}} g \Pi_{0}^{\prime} P_{\frac{u}{a_{n}}}^{k} P^{k} h\right]\right) d u \\
& +O\left(a_{n}^{-d-1-\gamma^{\prime}}\|g\|_{L^{q_{0}}\left(\mu_{\Delta}\right)} \sup _{t}\left\|P_{t}^{k} P^{k} h\right\|_{\mathcal{B}}\right) .
\end{aligned}
$$

If $k=0$, we go directly to (100). Otherwise we proceed as follows. Since $\left|e^{i x}-1-i x\right| \leq$ $2|x|^{1+\widetilde{\gamma}}$ for $\tilde{\gamma}:=\min \left(1, \frac{p\left(q_{0}-1\right)}{q_{0}}-1, \frac{p}{q_{1}}-1\right) \in(0,1]$ (since $\frac{1}{q_{0}}+\frac{1}{p}<1$ and $q_{1}<p$ ),

$$
\mathbb{E}_{\mu_{\Delta}}\left[\left(e^{i \frac{u}{a_{n}} \cdot \hat{\kappa}_{k}}-1-i \frac{u}{a_{n}} \cdot \hat{\kappa}_{k}\right) g\right]=O\left(\frac{1}{a_{n}^{1+\widetilde{\gamma}}} \mathbb{E}_{\mu_{\Delta}}\left[\left|u \cdot \hat{\kappa}_{k}\right|^{1+\widetilde{\gamma}}|g|\right]\right)
$$




$$
=O\left(\frac{|u|^{1+\widetilde{\gamma}}}{a_{n}^{1+\widetilde{\gamma}}}\left\|\left|\hat{\kappa}_{k}\right|^{1+\tilde{\gamma}}\right\|_{L^{\frac{q_{0}}{q_{0}-1}\left(\mu_{\Delta}\right)}}\|g\|_{L^{q_{0}}\left(\mu_{\Delta}\right)}\right)
$$

due to the Hölder inequality, noting that $\frac{q_{0}}{q_{0}-1}(1+\tilde{\gamma}) \leq p$. Thus, using (97) with $s=1+\tilde{\gamma}$ and $r=\frac{q_{0}}{q_{0}-1}$, we obtain

$$
\begin{aligned}
& \mathbb{E}_{\mu_{\Delta}}\left[\left(e^{i \frac{u}{a_{n}} \cdot \hat{\kappa}_{k}}-1-i \frac{u}{a_{n}} \cdot \hat{\kappa}_{k}\right) g\right]=O\left(|u|^{1+\widetilde{\gamma}} \frac{k^{1+\tilde{\gamma}}\|\hat{\kappa}\|^{1+\tilde{\gamma}} \frac{q_{0}(1+\tilde{\gamma})}{L_{0}^{-1}\left(\mu_{\Delta}\right)}\|g\|_{\mathbb{L}^{q_{0}}\left(\mu_{\Delta}\right)}}{a_{n}^{1+\widetilde{\gamma}}}\right), \\
& \mathbb{E}_{\mu_{\Delta}}\left[\left(e^{i \frac{u}{a_{n}} \cdot \hat{\kappa}_{k}}-1-i \frac{u}{a_{n}} \cdot \hat{\kappa}_{k}\right) P^{k} h\right]=O\left(|u|^{1+\widetilde{\gamma}} \frac{k^{1+\widetilde{\gamma}}\left\|\hat{\kappa}^{1+\widetilde{\gamma}}\right\|_{L^{q_{1}}\left(\mu_{\Delta}\right)}\left\|P^{k} h\right\|_{\mathbb{L}^{p_{1}}\left(\mu_{\Delta}\right)}}{a_{n}^{1+\widetilde{\gamma}}}\right),
\end{aligned}
$$

noticing that $(1+\tilde{\gamma}) \frac{q_{0}}{q_{0}-1} \leq p$ and $(1+\tilde{\gamma}) q_{1} \leq p$. Therefore

$$
\begin{aligned}
& \mathbb{E}_{\mu_{\Delta}}\left[h \mathbf{1}_{\left\{\hat{\kappa}_{n} \circ f^{k}=N\right\}} g \circ f^{n}\right]=\frac{1}{\left(2 \pi a_{n}\right)^{d}} \mathbb{E}_{\mu_{\Delta}}[g] \mathbb{E}_{\mu_{\Delta}}[h] \int_{\left[-\beta a_{n}, \beta a_{n}\right]^{d}} e^{-i \frac{u}{a_{n}} \cdot N} \lambda_{u / a_{n}}^{n-2 k} d u \\
& \quad+\frac{1}{(2 \pi)^{d}\left(a_{n}\right)^{d+1}} \int_{\left[-\beta a_{n}, \beta a_{n}\right]^{d}} u \cdot D_{n, k}(g, h) e^{-i \frac{u}{a_{n}} \cdot N} \lambda_{u / a_{n}}^{n-2 k} d u \\
& \quad+O\left(a_{n}^{-d-1} / \log n\|g\|_{L^{q_{0}}\left(\mu_{\Delta}\right)} \sup _{t}\left\|P_{t}^{k} P^{k} h\right\|_{\mathcal{B}}\right),
\end{aligned}
$$

with $D_{n, k}(g, h):=\left(\mathbb{E}_{\mu_{\Delta}}\left[i \hat{\kappa}_{k}\left(g \Pi_{0}(h)+\Pi_{0}(g) P^{k} h\right)+e^{i \frac{u}{a_{n}} \cdot \hat{\kappa}_{k} \circ f^{k}} g \Pi_{0}^{\prime} P_{\frac{u}{a_{n}}}^{k} P^{k} h\right]\right)$, since $k^{1+\widetilde{\gamma}} \ll a_{n}^{\tilde{\gamma}} / \log n$ due to our assumption on $k$. Recall that $\Pi_{0}^{\prime} \in \mathcal{L}\left(\mathcal{B}, L^{p_{0}}\left(\mu_{\Delta}\right)\right)$. Choosing $\gamma_{2}=\min \left(1, \frac{p_{0}}{q_{0}}\right) \in(0,1]$ (since $\left.q_{0}<\infty\right)$ so that $1 \leq \gamma_{2} q_{0} \leq p_{0}$,

$$
\begin{aligned}
\left|\mathbb{E}_{\mu_{\Delta}}\left[\left(e^{i \frac{u}{a_{n}} \cdot \hat{\kappa}_{k} \circ f^{k}}-1\right) g \Pi_{0}^{\prime} P_{u / a_{n}}^{k} P^{k} h\right]\right| & \leq 2\left|u / a_{n}\right|^{\gamma_{2}} \mathbb{E}_{\mu_{\Delta}}\left[\left|\hat{\kappa}_{k}\right|^{\gamma_{2}} \Pi_{0}^{\prime} P_{u / a_{n}}^{k} P^{k} h\right] \\
& \leq 2\left|u / a_{n}\right|^{\gamma_{2}} k^{\gamma_{2}}\|\hat{\kappa}\|_{L^{\gamma_{2} q_{0}}\left(\mu_{\Delta}\right)}^{\gamma_{2}}\left\|\Pi_{0}^{\prime} P_{u / a_{n}}^{k} P^{k} h\right\|_{L^{p_{0}}\left(\mu_{\Delta}\right)},
\end{aligned}
$$

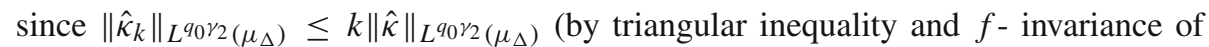
$\left.\mu_{\Delta}\right)$. So

$$
\begin{aligned}
& \mathbb{E}_{\mu_{\Delta}}\left(h \mathbf{1}_{\left\{\hat{S}_{n}=N\right\}} g \circ f^{n}\right)=\frac{1}{\left(2 \pi a_{n}\right)^{d}} \int_{\left[-\beta a_{n}, \beta a_{n}\right]^{d}} e^{-i \frac{u}{a_{n}} \cdot N} \lambda_{u / a_{n}}^{n-2 k} d u \mathbb{E}_{\mu_{\Delta}}[g] \mathbb{E}_{\mu_{\Delta}}[h] \\
& +\frac{1}{(2 \pi)^{d}\left(a_{n}\right)^{d+1}} \int_{\left[-\beta a_{n}, \beta a_{n}\right]^{d}} u e^{-i \frac{u}{a_{n}} \cdot N} \lambda_{u / a_{n}}^{n-2 k} \mathcal{E}_{k}\left(g, h, u / a_{n}\right) d u+O\left(a_{n}^{-d-1} / \log n\right),
\end{aligned}
$$

since $\left(k / a_{n}\right)^{\gamma_{2}} \ll(\log n)^{-1}$ and with

$$
\mathcal{E}_{k}(g, h, t):=\mathbb{E}_{\mu_{\Delta}}\left[g \Pi_{0}^{\prime} P_{t}^{k} P^{k} h\right]+i \mathbb{E}_{\mu_{\Delta}}\left[\hat{\kappa}_{k} g\right] \mathbb{E}_{\mu_{\Delta}}[h]+i \mathbb{E}_{\mu_{\Delta}}[g] \mathbb{E}_{\mu_{\Delta}}\left[\hat{\kappa}_{k} P^{k} h\right] .
$$

Therefore

$$
\mathbb{E}_{\mu_{\Delta}}\left(h \mathbf{1}_{\left\{\hat{S}_{n}=N\right\}} g \circ f^{n}\right)=\frac{1}{\left(2 \pi a_{n}\right)^{d}} \int_{\left[-\beta a_{n}, \beta a_{n}\right]^{d}} e^{-i \frac{u}{a_{n}} \cdot N} \lambda_{u / a_{n}}^{n-2 k} d u \mathbb{E}_{\mu_{\Delta}}[g] \mathbb{E}_{\mu_{\Delta}}[h]
$$




$$
\begin{aligned}
& +\frac{\mathcal{E}_{k}(g, h, 0)}{(2 \pi)^{d}\left(a_{n}\right)^{d+1}} \cdot \int_{\left[-\beta a_{n}, \beta a_{n}\right]^{d}} u e^{-i \frac{u}{a_{n}} \cdot N} \lambda_{u / a_{n}}^{n-2 k} d u+O\left(a_{n}^{-d-1} / \log n\right) \\
& +O\left(\frac{1}{(2 \pi)^{d}\left(a_{n}\right)^{d+1}} \int_{\left[-\beta a_{n}, \beta a_{n}\right]^{d}}|u| e^{-\frac{c_{0} \min \left(|u|^{2-\epsilon},|u|^{2+\epsilon}\right)}{2}}\left|\mathbb{E}_{\mu_{\Delta}}\left[g \Pi_{0}^{\prime}\left(P_{u / a_{n}}^{k} P^{k} h-P^{2 k} h\right)\right]\right| d u\right),
\end{aligned}
$$

due to (95). Due to (99) for $\ell=0$ and $\ell=1, \mathbb{E}_{\mu_{\Delta}}\left(h \mathbf{1}_{\left\{\hat{S}_{n}=N\right\}} g \circ f^{n}\right)$ is equal to

$$
\begin{aligned}
& \frac{1}{\left(2 \pi a_{n}\right)^{d}}\left(I_{0}\left(\frac{N}{a_{n}}\right)-\frac{1}{2} I_{2}\left(\frac{N}{a_{n}}\right) \frac{\log \log n}{\log n}+O\left(\frac{1}{\log n}\right)\right) \mathbb{E}_{\mu_{\Delta}}[g] \mathbb{E}_{\mu_{\Delta}}[h] \\
& \quad+\frac{\mathcal{E}_{k}(g, h, 0)}{a_{n}^{d+1}} \mathbb{E}_{\mu_{\Delta}}\left(I_{1}\left(\frac{N}{a_{n}}\right)-\frac{1}{2} I_{3}\left(\frac{N}{a_{n}}\right) \frac{\log \log n}{\log n}\right)+O\left(a_{n}^{-d-1} / \log n\right) \\
& \quad+O\left(\frac{1}{(2 \pi)^{d}\left(a_{n}\right)^{d+1}} \int_{\left[-\beta a_{n}, \beta a_{n}\right]^{d}}|u| e^{-\frac{c_{0} \min \left(|u|^{2-\epsilon},|u|^{2+\epsilon}\right)}{2}}\left|\mathbb{E}_{\mu_{\Delta}}\left[g \Pi_{0}^{\prime}\left(P_{u / a_{n}}^{k} P^{k} h-P^{2 k} h\right)\right]\right| d u\right),
\end{aligned}
$$

with $I_{2 k+1}(x):=\frac{1}{(2 \pi)^{d}} \int_{\mathbb{R}^{d}} u(A u \cdot u)^{k} e^{-i u \cdot x} e^{-\frac{1}{2} A u \cdot u} d u=i(-1)^{k} \frac{\left(\Delta_{1, k} \Phi\right)\left(A^{-\frac{1}{2}} x\right)}{\sqrt{\operatorname{det} A}}$, with $\Delta_{1, k} \Phi(x):=A^{-\frac{1}{2}}\left(\sum_{j=1}^{d} \frac{\partial^{2 k+1}}{\partial x_{i} \partial^{2 k} x_{j}} \Phi(x)\right)_{i=1, \ldots, d}$, which leads to (90), ending the proof of the Proposition.

7.2. Decorrelation expansion for $\mathbb{Z}^{d}$-extensions. We are now interested in decorrelation expansion for $\mathbb{Z}^{d}$-extensions satisfying the set up of Proposition 7.1. In this subsection, we consider the $\mathbb{Z}^{d}$-extension $(\Omega, v, S)$ of $\left(\Delta, f, \mu_{\Delta}\right)$ with step function $\hat{\kappa}: \Delta \rightarrow \mathbb{Z}^{d}$, where $\Omega=\Delta \times \mathbb{Z}^{d}$, the transformation $S$ is defined by $S(x, L)=(f x, L+\hat{\kappa}(x))$ and preserves the infinite measure $v=\mu_{\Delta} \otimes \mathfrak{m}_{d}$ with $\mathfrak{m}_{d}$ being the counting measure on $\mathbb{Z}^{d}$.

Corollary 7.3. Assume (84)-(86). Suppose that (87) holds with $\gamma>0$. Let $\phi, \psi: \Delta \times$ $\mathbb{Z}^{d} \rightarrow \mathbb{R}$ be two observables such that $\sum_{N \in \mathbb{Z}^{d}}\left[\|\phi(\cdot, N)\|_{\mathcal{B}}+\|\psi(\cdot, N)\|_{L^{q_{2}}\left(\mu_{\Delta}\right)}\right]<\infty$ and $\sum_{N \in \mathbb{Z}^{d}}|N|^{\gamma}\left[\left|\int_{\Delta} \phi(\cdot, N) d \mu\right|+\left|\int_{\Delta} \psi(\cdot, N) d \mu\right|\right]<\infty$. Then

$$
\int_{\Omega} \phi \cdot \psi \circ S^{n} d \nu=\frac{I_{0}(0)}{(n \log n)^{\frac{d}{2}}}\left(1-\frac{d}{2} \frac{\log \log n}{\log n}\right) \int_{\Omega} \phi d \mu \int_{\Omega} \psi d \mu+O\left(\frac{1}{(n \log n)^{\frac{d}{2}} \log n}\right) .
$$

Suppose that (88) (instead of (87)) holds with $\gamma^{\prime}>0$ and assume that there exists $\gamma_{0}>0$ such that $\sum_{N \in \mathbb{Z}^{d}}\left(1+|N|^{\gamma_{0}}\right)\left(\|\psi(\cdot, N)\|_{L^{q_{0}}\left(\mu_{\Delta}\right)}+\|\phi(\cdot, N)\|_{\mathcal{B}}\right)<\infty$. Then

$$
\begin{aligned}
& \int_{\Omega} \phi \cdot \psi \circ S^{n} d v=\frac{I(0)}{(n \log n)^{\frac{d}{2}}}\left(1-\frac{d \log \log n+O(1)}{2 \log n}\right) \int_{\Omega} \phi d \nu \\
& \int_{\Omega} \psi d v+O\left(\frac{1}{(n \log n)^{\frac{d+1}{2}} \log n}\right) .
\end{aligned}
$$

Proof. Applying Proposition 7.1 to the couples $\left(\phi\left(\cdot, N_{1}\right), \psi\left(\cdot, N_{2}\right)\right)$ with $k=0$ leads to

$$
\int_{\Omega} \phi \cdot \psi \circ S^{n} d \nu=\sum_{N_{1}, N_{2} \in \mathbb{Z}^{d}} \mathbb{E}_{\mu_{\Delta}}\left[\phi\left(\cdot, N_{1}\right) \mathbf{1}_{\left\{\hat{\kappa}_{n}=N_{2}-N_{1}\right\}} \psi\left(f^{n}(\cdot), N_{2}\right)\right]
$$




$$
\begin{aligned}
& =\sum_{N_{1}, N_{2} \in \mathbb{Z}^{d}} \frac{1}{a_{n}^{d}} \mathbb{E}_{\mu_{\Delta}}\left[\phi\left(\cdot, N_{1}\right)\right] \mathbb{E}_{\mu_{\Delta}}\left[\phi\left(\cdot, N_{2}\right)\right]\left(I_{0}\left(\frac{N_{2}-N_{1}}{a_{n}}\right)-\frac{I_{2}\left(\frac{N_{2}-N_{1}}{a_{n}}\right)}{2} \frac{\log \log n}{\log n}\right) \\
& \quad+O\left(\frac{1}{a_{n}^{d} \log n}\right) .
\end{aligned}
$$

Thus $\int_{\Omega} \phi . \psi \circ S^{n} d v=\frac{1}{a_{n}^{d}} \sum_{N_{1}, N_{2} \in \mathbb{Z}^{d}} \int_{M_{N_{1}}} \psi d \mu \int_{M_{N_{2}}} \phi d \mu I_{0}(0)\left(1-\frac{d}{2} \frac{\log \log n}{\log n}\right)+$ $O\left(\frac{1}{a_{n}^{d} \log n}\right)$. We used the fact that for every $\gamma \in(0,2]$, there exists $C_{\gamma}$ such that, for every $X \in \mathbb{R}^{2},\left|I_{0}(X)-I_{0}(0)\right|+\left|I_{2}(X)-I_{2}(0)\right| \leq C_{\gamma}|X|^{\gamma}$, that $\left|N_{2}-N_{1}\right| \leq\left|N_{2}\right|+\left|N_{1}\right|$ combined with our assumption on $\phi$ and $\psi$. Setting $\tilde{I}_{k}(n, x):=I_{k}(x)-\frac{I_{k+2}(x)}{2} \frac{\log \log n}{\log n}$ and applying the second point of Proposition 7.1, we obtain that $\int_{\Omega} \phi . \psi \circ S^{n} d v$ is equal to

$$
\begin{aligned}
& \frac{1}{a_{n}^{d}} \sum_{N_{1}, N_{2} \in \mathbb{Z}^{d}} \mathbb{E}_{\mu_{\Delta}}\left[\phi\left(\cdot, N_{1}\right)\right] \mathbb{E}_{\mu_{\Delta}}\left[\psi\left(\cdot, N_{2}\right)\right]\left(\tilde{I}_{0}\left(n, \frac{N_{2}-N_{1}}{a_{n}}\right)+O\left(\frac{1}{\log n}\right)\right) \\
& \quad+\frac{1}{\left(a_{n}\right)^{d+1}} \sum_{N_{1}, N_{2} \in \mathbb{Z}^{d}} \mathbb{E}_{\mu_{\Delta}}\left[\psi\left(\cdot, N_{2}\right) \Pi_{0}^{\prime} \phi\left(\cdot, N_{1}\right)\right] \tilde{I}_{1}\left(n, \frac{N_{2}-N_{1}}{a_{n}}\right)+O\left(\frac{1}{a_{n}^{d+1} \log n}\right) \\
& =\frac{I_{0}(0)}{a_{n}^{d}} \sum_{N_{1}, N_{2} \in \mathbb{Z}^{d}} \mathbb{E}_{\mu_{\Delta}} \\
& \quad\left[\phi\left(\cdot, N_{1}\right)\right] \mathbb{E}_{\mu_{\Delta}}\left[\psi\left(\cdot, N_{2}\right)\right]\left(1-\frac{d}{2} \frac{\log \log n}{\log n}+O\left(\frac{1}{\log n}\right)\right)+O\left(\frac{1}{a_{n}^{d+1} \log n}\right),
\end{aligned}
$$

where we used the same argument as before with $\gamma \in(1,2]$ combined with the fact that $I_{1}$ and $I_{3}$ are uniformly $\gamma_{0}$-Hölder with $\gamma_{0} \in(0,1)$ and that $I_{1}(0)=I_{3}(0)=0$.

Observe that when $\phi$ or $\psi$ has null integral, then Corollary 7.3 only provides an upper bound (given by the term in $O(\cdot)$ ). Nevertheless, the method we used to establish Proposition 7.1 and Corollary 7.3 enables the establishment of explicit decorrelation rates

for some specific but natural null integral observables of the $\mathbb{Z}^{d}$-extension, including coboundaries. Before stating these decorrelation results, let us introduce the following notations. Set

$$
\begin{aligned}
I_{\ell_{1}, \ell_{2}, N}(x) & :=\frac{1}{(2 \pi)^{d}} \int_{\mathbb{R}^{d}}(-i u \cdot N)^{\ell_{1}}(-A u \cdot u)^{\ell_{2}} e^{-i u \cdot x} e^{-\frac{1}{2} A u \cdot u} d u \\
& =\frac{1}{(2 \pi)^{d} \sqrt{\operatorname{det} A}} \int_{\mathbb{R}^{d}}\left(-i u \cdot A^{-1 / 2} N\right)^{\ell_{1}}(-u \cdot u)^{\ell_{2}} e^{-i u \cdot A^{-1 / 2} x} e^{-\frac{1}{2} u \cdot u} d u \\
& =\frac{\Delta_{\ell_{1}, \ell_{2}} \Phi\left(A^{-\frac{1}{2}} x, N\right)}{\sqrt{\operatorname{det} A}}
\end{aligned}
$$

with $\Delta_{\ell_{1}, \ell_{2}} \Phi(x, N):=\left(A^{-\frac{1}{2}} N \cdot \nabla\right)^{\ell_{1}} \Delta^{\ell_{2}} \Phi\left(A^{-1 / 2} x\right)$ with $\Phi(x)=\frac{e^{-\frac{1}{2} x \cdot x}}{(2 \pi)^{d / 2}}$ and where $\nabla$ and $\Delta$ are the usual differential gradient and Laplacian operators. Recall that, for $\psi: \mathbb{R}^{d} \rightarrow \mathbb{C} ; \nabla \psi=\left(\frac{\partial}{\partial x_{i}} \psi\right)_{i=1, \ldots, d}$ and that $\Delta \psi=(\nabla \cdot \nabla) \psi=\sum_{i=1}^{d} \frac{\partial^{2}}{\partial^{2} x_{i}} \psi$.

Proposition 7.4. Assume assumptions of Proposition 7.1 with (87). 
(A) (Coboundary) If $\phi(x, \ell)=h_{\ell}(x)$ and $\psi(x, \ell)=g_{\ell}(x)$, with $\sum_{N \in \mathbb{Z}^{d}}$ $\left(\left\|g_{N}\right\|_{L^{q_{2}}\left(\mu_{\Delta}\right)}+\left\|h_{N}\right\|_{\mathcal{B}}\right)<\infty$, and $\sum_{N \in \mathbb{Z}^{d}}|N|^{\delta}\left(\left\|g_{N}\right\|_{L^{1}\left(\mu_{\Delta}\right)}+\left\|h_{N}\right\|_{L^{1}\left(\mu_{\Delta}\right)}\right)<\infty$ for some $\delta \in(0,1)$, then, for all integer $m \geq 1, \int_{\Omega} \phi \circ(i d-S)^{m} . \psi \circ S^{n} d v$ is equal to

$$
\begin{aligned}
& \frac{(\log n)^{m}+m \log \log n(\log n)^{m-1}}{2^{m} a_{n}^{d+2 m}}\left(I_{0, m, 0}(0)+I_{0, m+1,0}(0) \frac{\log \log n}{2 \log n}\right) \\
& \times \int_{\Omega} g d v \int_{\Omega} h d v+O\left(a_{n}^{-d-2 m}(\log n)^{m-1}\right) .
\end{aligned}
$$

(B) Let $N \in \mathbb{Z}^{d}$ be fixed. If $\phi(x, \ell)=h(x)\left(1_{\ell=N}+1_{\ell=-N}-2 \times 1_{\ell=0}\right)$ and $\psi(x, \ell)=g(x)$ with $g \in L^{q_{2}}\left(\mu_{\Delta}\right)$ and $h \in \mathcal{B}$, then

$$
\begin{aligned}
\int_{\Omega} \phi . \psi \circ S^{n} d v= & -\frac{A^{-1} N \cdot N}{a_{n}^{d+2} \sqrt{(2 \pi)^{d} \operatorname{det}(A)}} \mathbb{E}_{\mu_{\Delta}}[h] \mathbb{E}_{\mu_{\Delta}}[g]\left(1-\frac{(d+2) \log \log n}{2 \log n}+O\left((\log n)^{-1}\right)\right) \\
& +O\left(a_{n}^{-d-2-\min (\delta, \gamma)}\right) .
\end{aligned}
$$

Let us observe that item (B) of Proposition 7.4 (with $h=g=1_{\Delta}$ ) implies in particular that $\mu_{\Delta}\left(\hat{\kappa}_{n}=1\right)+\mu_{\Delta}\left(\hat{\kappa}_{n}=-1\right)-2 \mu_{\Delta}\left(\hat{\kappa}_{n}=0\right) \approx a_{n}^{-d-2}$, as $n \rightarrow+\infty$.

Proof of Proposition 7.4. Set $J_{\ell_{1}, \ell_{2}, n, N, N_{0}}:=\frac{1}{(2 \pi)^{d}} \int_{-\left[\beta a_{n}, \beta a_{n}\right]^{d}} e^{-i u \cdot N / a_{n}}\left(-i u \cdot N_{0}\right)^{\ell_{1}}$ $(-A u \cdot u)^{\ell_{2}} \lambda_{u / a_{n}}^{n-m} d u$, with $m=0$ in item (B). Observe first that, due to (98)

$$
\forall \delta_{0} \in(0,1], \quad J_{\ell_{1}, \ell_{2}, n, N, N_{0}}:=J_{\ell_{1}, \ell_{2}, n, 0, N_{0}}+O\left(\frac{N_{0}^{\ell_{1}} N^{\delta_{0}}}{a_{n}^{\delta_{0}}}\right)
$$

using $\left|e^{i x}-1\right| \leq 2|x|^{\delta_{0}}$, and, moreover, due to (99)

$$
J_{\ell_{1}, \ell_{2}, n, 0, N_{0}}=I_{\ell_{1}, \ell_{2}, N_{0}}(0)+I_{\ell_{1}, \ell_{2}+1, N_{0}}(0) \frac{\log \log n}{2 \log n}+O\left(\frac{N_{0}^{\ell_{1}}}{\log n}\right),
$$

for every $\delta \in[0,1]$. Note that $I_{\ell_{1}, \ell_{2}, N}$ has same parity as $\ell_{1}$ and thus that $I_{\ell_{1}, \ell_{2}, N}(0)=0$ if $\ell_{1}$ is an odd number.

- Assume the assumptions of (A). Then

$$
\begin{gathered}
\int_{\Omega} \phi \circ(i d-S)^{m} \cdot \psi \circ S^{n} d v=\sum_{r=0}^{m} \frac{m !(-1)^{r}}{r !(m-r) !} \int_{\Omega} \phi \circ S^{r} . \psi \circ S^{n} d \nu \\
=\sum_{N_{1}, N_{2} \in \mathbb{Z}^{d}} \sum_{r=0}^{m} \frac{m !(-1)^{r}}{r !(m-r) !} \mathbb{E}_{\mu_{\Delta}}\left[h_{N_{1}} 1_{\left\{\hat{\kappa}_{n-r}=N_{2}-N_{1}\right\}} g_{N_{2}} \circ f^{n-r}\right] .
\end{gathered}
$$

Thus, due to (94) with $k=0$ and since $\sum_{r=0}^{m} \frac{m !(-1)^{r} \lambda_{t}^{n-r}}{r !(m-r) !}=(-1)^{m} \lambda_{t}^{n-m}\left(1-\lambda_{t}\right)^{m}$, $\int_{\Omega} \phi \circ(i d-S)^{m} \cdot \psi \circ S^{n} d v$ is equal to 


$$
\begin{aligned}
& \frac{(-1)^{m}}{(2 \pi)^{d} a_{n}^{d}} \sum_{N_{1}, N_{2} \in \mathbb{Z}^{d}} \int_{\left[-\beta a_{n}, \beta a_{n}\right]^{d}} e^{-i u \cdot\left(N_{2}-N_{1}\right) / a_{n}} \lambda_{u / a_{n}}^{n-m}\left(1-\lambda_{u / a_{n}}\right)^{m} \mathbb{E}_{\mu_{\Delta}}\left[g_{N_{2}} \Pi_{u / a_{n}} h_{N_{1}}\right] d u \\
& \quad+O\left(\sum_{N_{1}, N_{2} \in \mathbb{Z}^{d}} \theta^{n}\left\|g_{N_{2}}\right\|_{L^{q_{1}}\left(\mu_{\Delta}\right)}\left\|h_{N_{1}}\right\|_{\mathcal{B}}\right)
\end{aligned}
$$

that can be rewritten

$$
\begin{aligned}
& \frac{(-1)^{m}}{(2 \pi)^{d} a_{n}^{d+2 m}} \sum_{N_{1}, N_{2} \in \mathbb{Z}^{d}} \int_{\left[-\beta a_{n}, \beta a_{n}\right]^{d}} e^{-i u \cdot\left(N_{2}-N_{1}\right) / a_{n}} \lambda_{u / a_{n}}^{n-m}\left(A u \cdot u \log \left(\left|a_{n} / u\right|\right)\right)^{m} \mathbb{E}_{\mu_{\Delta}}\left[g \Pi_{u / a_{n}} h\right] d u \\
& +O\left(\sum_{N_{1}, N_{2} \in \mathbb{Z}^{d}} a_{n}^{-d-2 m}\left\|g_{N_{2}}\right\|_{L^{q_{1}}\left(\mu_{\Delta}\right)}\left\|h_{N_{1}}\right\|_{\mathcal{B}}\left(1+\int_{\left[-\beta a_{n}, \beta a_{n}\right]^{d}} e^{-c_{0} \min \left(|u|^{2-\epsilon},|u|^{2+\epsilon}\right)}|u|^{2 m}\left(\log \left(\left|a_{n} / u\right|\right)\right)^{m-1} d u\right)\right)
\end{aligned}
$$

where we used (86) and (95). Now writing $\log \left(\left|a_{n} / u\right|\right)=\log a_{n}+O(\log |u|)$ and using (87), we obtain that $\int_{\Omega} \phi \circ(i d-S)^{m} . \psi \circ S^{n} d \nu$ is equal to

$$
\sum_{N_{1}, N_{2} \in \mathbb{Z}^{d}} \frac{\left(\log a_{n}\right)^{m}}{a_{n}^{d+2 m}} J_{0, m, n, 0,0} \mathbb{E}_{\mu_{\Delta}}\left[g_{N_{2}}\right] \mathbb{E}_{\mu_{\Delta}}\left[h_{N_{1}}\right]+O\left(a_{n}^{-d-2 m}\left(\log a_{n}\right)^{m-1} \sum_{N_{1}, N_{2}}\left\|g_{N_{2}}\right\|_{L^{q_{2}}\left(\mu_{\Delta}\right)}\left\|h_{N_{1}}\right\|_{\mathcal{B}}\right),
$$

due to (102) with $\delta_{0}=\delta$, to $\Pi_{0}=\mathbb{E}_{\mu_{\Delta}}[\cdot] 1_{\Delta}$ and to our summability assumptions. This ends the proof of (A) since $\int_{\Omega} \phi d v=\sum_{N \in \mathbb{Z}^{d}} \mathbb{E}_{\mu_{\Delta}}\left[h_{N}\right]$ and $\int_{\Omega} \psi d v=$ $\sum_{N \in \mathbb{Z}^{d}} \mathbb{E}_{\mu_{\Delta}}\left[g_{N}\right]$.

- Assume the assumptions of (B). Then, due to (94) with $k=0$, we obtain

$$
\begin{aligned}
& \int_{\Omega} \phi \cdot \psi \circ S^{n} d v=\mathbb{E}_{\mu_{\Delta}}\left[h\left(1_{\left\{\hat{\kappa}_{n} \circ f^{k}=N\right\}}+1_{\left\{\hat{K}_{n} \circ f^{k}=N\right\}}-2 \times 1_{\left\{\hat{K}_{n} \circ f^{k}=0\right\}}\right) g \circ f^{n}\right] \\
& \quad=\frac{1}{(2 \pi)^{d} a_{n}^{d}} \int_{\left[-\beta a_{n}, \beta a_{n}\right]^{d}}\left(e^{-i u \cdot N / a_{n}}+e^{i u \cdot N / a_{n}}-2\right) \lambda_{u / a_{n}}^{n} \mathbb{E}_{\mu_{\Delta}}\left[g \Pi_{u / a_{n}} h\right] d t+O\left(\theta^{n}\|g\|_{L^{q_{1}}\left(\mu_{\Delta}\right)}\|h\|_{\mathcal{B}}\right) .
\end{aligned}
$$

Therefore $\int_{\Omega} \phi . \psi \circ S^{n} d v$ is equal to

$$
\begin{aligned}
& \frac{1}{(2 \pi)^{d} a_{n}^{d+2}} \int_{\left[-\beta a_{n}, \beta a_{n}\right]^{d}}(i u \cdot N)^{2} \lambda_{u / a_{n}}^{n} \mathbb{E}_{\mu_{\Delta}}\left[g \Pi_{u / a_{n}} h\right] d t+O\left(a_{n}^{-d-4}\|g\|_{L^{q_{1}}\left(\mu_{\Delta}\right)}\|h\|_{\mathcal{B}}\right) \\
& =\frac{1}{a_{n}^{d+2}} \mathbb{E}_{\mu_{\Delta}}[g] \mathbb{E}_{\mu_{\Delta}}[h] J_{2,0, n, 0, N}+O\left(a_{n}^{-d-2-\gamma}\|g\|_{L^{q_{1}\left(\mu_{\Delta}\right)}}\|h\|_{\mathcal{B}}\right),
\end{aligned}
$$

due to (87). We end the proof of (B) by using (103) combined with

$$
I_{2,0, N}(0)=-\frac{A^{-1} N \cdot N}{\sqrt{(2 \pi)^{d} \operatorname{det}(A)}}, \quad I_{2,1, N}(0)=\frac{(d+2) A^{-1} N \cdot N}{\sqrt{(2 \pi)^{d} \operatorname{det}(A)}} .
$$


7.3. Decorrelation for the $\mathbb{Z}^{d}$-periodic billiard map. We consider the setting of Sect. 2 with (12). Recall that the billiard map $T$ can be represented as the $\mathbb{Z}^{d}$-extension of $(\bar{M}, \bar{T}, \bar{\mu})$ with step function $\kappa: \bar{M} \rightarrow \mathbb{Z}^{d}$ (the step function of the one-dimensional case corresponding to the first coordinate of the step function of the two dimensional case). Therefore these two billiard maps can be represented as the $\mathbb{Z}^{d}$-extension of $(\bar{M}, \bar{\mu}, \bar{T})$ by a step function $\kappa: \bar{M} \rightarrow \mathbb{Z}^{d}$ with $d \in\{1,2\}$ such that there exists $\hat{\kappa}: \Delta \rightarrow \mathbb{Z}^{d}$ satisfying $\hat{\kappa} \circ \mathfrak{p}_{2}=\kappa \circ \mathfrak{p}_{1}$. We treat together these two models in the following. We consider the Banach spaces $\mathcal{B}_{0}$ and $\mathcal{B}$ defined in Sect. 5.1. As recalled in Sect. 5.1, with these choices, (15) and (16) hold. Since $\mathcal{B}_{0}$ is continuously embedded in $\mathcal{B},(84)$ and (85) holds true with the Banach space $\mathcal{B}_{0}$ and for any $p_{1} \in[1,+\infty)$. Moreover (86) has been proved in Proposition 3.3 and, due to Proposition 5.3 (the fact that the symmetric matrix is invertible follows from the assumption of total dimension of the horizon), (88) (and so holds true (87) with any $\gamma \in(0,1])$ for $\mathcal{B}_{0}$ for any $p_{0} \in\left(1, \frac{4}{3}\right)$.

Remark 7.5. The second item of Proposition 7.1 applies providing a mixing local limit theorem for $\mathbb{E}_{\mu}\left(\phi \mathbf{1}_{\left\{\kappa_{n}=N\right\}} \psi \circ \bar{T}^{n}\right)$ with $\phi$ and $\psi$ both constant on stable curves, with $\phi$ dynamically Hölder and $\psi \in L^{q_{0}}(\bar{\mu})$. Corollary 7.3 applies analogously.

Our goal is to prove Theorems 2.2 and 2.4 for general dynamically Hölder observables. To this end, we will use approximations by functions on $\Delta$ and Proposition 7.1 with $k=k(n) \rightarrow+\infty$. Recall that we have defined $\xi_{k}^{k^{\prime}}, \xi_{k}^{\infty}$ before Theorem 2.2. For any $\phi: M \rightarrow \mathbb{C}$ or $\phi: \bar{M} \rightarrow \mathbb{C}$ and $-\infty<k \leq k^{\prime} \leq \infty$, we define the local variation $\omega_{k}^{k^{\prime}}(\phi, x):=\sup _{y \in \xi_{k}^{k^{\prime}}(x)}|\phi(x)-\phi(y)|$, where $\xi_{k}^{k^{\prime}}(x)$ is the element of $\xi_{k}^{k^{\prime}}$ containing $x$. We start with a local limit theorem (generalizing Theorem 2.2). Set $\kappa_{n}:=\sum_{k=0}^{n-1} \kappa \circ \bar{T}^{k}$.

Theorem 7.6. (a) Let $(k(n))_{n}$ be a sequence of integers diverging to $+\infty$ such that $k(n)=$ $O\left(a_{n} / \log n\right)$. Let $N \in \mathbb{Z}^{d}$ and $\phi, \psi: \bar{M} \rightarrow \mathbb{C}$ be two bounded measurable functions, then

$$
\begin{aligned}
& \int_{\bar{M}} \phi 1_{\left\{\kappa_{n}=N\right\}} \psi \circ \bar{T}^{n} d \bar{\mu}=\frac{1}{a_{n}^{d}}\left(I_{0}\left(\frac{N}{a_{n}}\right)-\frac{1}{2} I_{2}\left(\frac{N}{a_{n}}\right) \frac{\log \log n}{\log n}\right) \int_{\bar{M}} \phi d \bar{\mu} \int_{\bar{M}} \psi d \bar{\mu} \\
& \left.\left.\quad+O\left(a_{n}^{-d} I_{0}\left(\frac{N}{2 a_{n}}\right)\left(\|\phi\|_{\infty}\left\|\omega_{-k(n)}^{\infty}(\psi, \cdot)\right\|_{L^{1}(\bar{\mu})}\right)+\|\psi\|_{\infty}\left\|\omega_{-k(n)}^{k(n)}(\phi, \cdot)\right\|_{L^{1}(\bar{\mu})}\right)\right)+\frac{\|\phi\|_{\infty}\|\psi\|_{\infty}}{a_{n}^{d} \log n}\right),
\end{aligned}
$$

uniformly in $N \in \mathbb{Z}^{d}$. In particular, if $\left.\lim _{k \rightarrow+\infty} \| \omega_{-k}^{k}(\phi, \cdot)+\omega_{-k}^{\infty}(\psi, \cdot)\right) \|_{L^{1}(\bar{\mu})}=0$, then the error term in (106) is in $o\left(a_{n}^{-d}\right)$, and if $\int_{\bar{M}}\left(\omega_{-k(n)}^{k(n)}(\phi, \cdot)+\omega_{-k(n)}^{\infty}(\psi, \cdot)\right) d \bar{\mu}=$ $O\left((\log n)^{-1}\right)$, then the error in (106) is in $O\left(\frac{1}{a_{n}^{d} \log n}\right)$.

(b) Assume that $k(n)=O\left(a_{n}^{\frac{1}{2}-u}\right)$ for some $u \in(0,1)$ and that there exists $p_{2}>2$ such that, for $h \in\{\phi, \psi\},\left\|\omega_{-k(n)}^{k(n)}(h, \cdot)\right\|_{\infty}=O\left((\log n)^{-1}\right)$, and $\left\|\omega_{-k(n)}^{k(n)}(h, \cdot)\right\|_{L^{1}(\bar{\mu})}=$ $O\left(\left(a_{n} \log n\right)^{-1}\right)$ and $\left\|\omega_{-k(n)}^{k(n)}(h, \cdot)\right\|_{L^{p_{2}(\bar{\mu})}}=o\left(k(n)^{-1} / \log n\right)$ and $\sum_{j \geq k(n)} \| \omega_{-j}^{j}$ $(h, \cdot) \|_{L^{p_{2}}(\bar{\mu})}=O\left((\log n)^{-1}\right)$, then the numeric series $A_{+}(\phi):=\sum_{j \geq 0} \mathbb{E}_{\bar{\mu}}\left[\kappa \circ \bar{T}^{j} . \phi\right]$ and $A_{-}(\psi):=\sum_{j \leq-1} \mathbb{E}_{\bar{\mu}}\left[\kappa \circ \bar{T}^{j} . \psi\right]$ converge absolutely and $\int_{\bar{M}} \phi 1_{\left\{\kappa_{n}=N\right\}} \psi \circ$ $\bar{T}^{n} d \bar{\mu}$ is equal to 


$$
\begin{aligned}
& \frac{1}{a_{n}^{d}}\left(I_{0}\left(\frac{N}{a_{n}}\right)-\frac{1}{2} I_{2}\left(\frac{N}{a_{n}}\right) \frac{\log \log n}{\log n}+O\left(\frac{1}{\log n}\right)\right) \mathbb{E}_{\bar{\mu}}[\phi] \mathbb{E}_{\bar{\mu}}[\psi] \\
& +i \frac{\mathbb{E}_{\bar{\mu}}[\psi] A_{+}(\phi)+\mathbb{E}_{\bar{\mu}}[\phi] A_{-}(\psi)}{a_{n}^{d+1}}\left(I_{1}\left(\frac{N}{a_{n}}\right)-\frac{I_{3}\left(\frac{N}{a_{n}}\right)}{2} \frac{\log \log n}{\log n}\right)+O\left(\frac{a_{n}^{-d-1}}{\log n}\right) .
\end{aligned}
$$

Proof. For the first assertion, we use the first part of Proposition 7.1 with $p>2$ (close to 2$), p_{1}=\infty\left(q_{1}=1\right), p_{0} \in(1,4 / 3)\left(p_{0}<p\right)$ and $q_{0}>4$ so that $\min \left(1, \frac{p}{q_{1}}, \frac{p}{p_{0}}\right)=1$. Moreover $k / a_{n} \ll(\log n)^{-1}$. We assume from now on, without loss of generality, that $\phi, \psi$ take their values in $\mathbb{R}$. To simplify notations, we write $k=k(n)$. We define $\phi^{(k)}$ and $\psi^{(k)}$ by

$$
\phi^{(k)}(\bar{x}):=\inf _{\xi_{-k}^{k}(x)} \phi_{+}-\sup _{\xi_{-k}^{k}(x)} \phi_{-} \text {and } \psi^{(k)}(\bar{x}):=\inf _{\xi_{-k}^{\infty}(x)} \psi_{+}-\sup _{\xi_{-k}^{\infty}(x)} \psi_{-},
$$

where $\phi_{+}=\max (\phi, 0), \psi_{+_{-}}=\max (\psi, 0), \phi_{-}=\max (-\phi, 0), \psi_{-}=\max (-\psi, 0)$. Observe that, for every $x \in \bar{M},\left|\phi^{(k)}(x)\right| \leq|\phi(x)|$ and $\left|\psi^{(k)}(x)\right| \leq|\psi(x)|$,

$$
\left|\phi^{(k)}(x)-\phi(x)\right| \leq \omega_{-k}^{k}(\phi, x) \leq 2\|\phi\|_{\infty} \text { and }\left|\psi^{(k)}(x)-\psi(x)\right| \leq \omega_{-k}^{\infty}(\psi, x) \leq 2\|\psi\|_{\infty} .
$$

Since $\phi^{(k)} \circ \bar{T}^{k}$ and $\psi^{(k)} \circ \bar{T}^{k}$ are constant on the stable curves, there exist $\tilde{\phi}^{(k)}, \tilde{\psi}^{(k)}$ : $\Delta \rightarrow \mathbb{C}$ such that $\tilde{\phi}^{(k)} \circ \mathfrak{p}_{2}=\phi^{(k)} \circ \bar{T}^{k} \circ \mathfrak{p}_{1}, \tilde{\psi}^{(k)} \circ \mathfrak{p}_{2}=\psi^{(k)} \circ \bar{T}^{k} \circ \mathfrak{p}_{1}$. Observe that $\left\|\tilde{\psi}^{(k)}\right\|_{\infty} \leq\left\|\psi^{(k)}\right\|_{\infty}$. Note that $\tilde{\phi}^{(k)}: \Delta \rightarrow \mathbb{C}$ is constant on balls of the form $\{y \in \Delta: s(x, y) \geq 2 k\}$ for every $x \in \Delta$. This will be useful to show that

$$
\sup _{u}\left\|P_{u}^{k} P^{k} \tilde{\phi}^{(k)}\right\|_{\mathcal{B}_{0}} \ll\left\|\phi^{(k)}\right\|_{\infty} .
$$

To this end, due to (32), for every $x_{1}, x_{2} \in \Delta$ such that $s_{0}\left(x_{1}, x_{2}\right) \geq 1$

$$
\begin{aligned}
& \left|P_{u}^{k} P^{k} \tilde{\phi}^{(k)}\left(x_{1}\right)-P_{u}^{k} P^{k} \tilde{\phi}^{(k)}\left(x_{2}\right)\right| \\
& \leq \sum_{\left(y_{1}, y_{2}\right): s\left(y_{1}, y_{2}\right) \geq 2 k+1, f^{2 k}\left(y_{i}\right)=x_{i}}\left|e^{-\alpha_{2 k}\left(y_{1}\right)+i u \hat{\kappa}_{k}\left(y_{1}\right)} \tilde{\phi}^{(k)}\left(y_{1}\right)-e^{-\alpha_{2 k}\left(y_{2}\right)+i u \hat{\kappa}_{k}\left(y_{2}\right)} \tilde{\phi}^{(k)}\left(y_{2}\right)\right| \\
& =\sum_{\left(y_{1}, y_{2}\right): s\left(y_{1}, y_{2}\right) \geq 2 k+1, f^{2 k}\left(y_{i}\right)=x_{i}}\left|\left(e^{-\alpha_{2 k}\left(y_{1}\right)}-e^{-\alpha_{2 k}\left(y_{2}\right)}\right) e^{i u \hat{\kappa}_{k}\left(y_{1}\right)} \tilde{\phi}^{(k)}\left(y_{1}\right)\right|,
\end{aligned}
$$

where we used the notation $\alpha_{m}:=\sum_{k=0}^{m-1} \alpha \circ f^{k}$. We end the proof of (110) by noticing that

$$
\begin{aligned}
\left|e^{-\alpha_{2 k}\left(y_{1}\right)}-e^{-\alpha_{2 k}\left(y_{2}\right)}\right| & \leq\left(e^{-\alpha_{2 k}\left(y_{1}\right)}+e^{-\alpha_{2 k}\left(y_{2}\right)}\right)\left|\alpha_{2 k}\left(y_{1}\right)-\alpha_{2 k}\left(y_{2}\right)\right| \\
& \leq\left(e^{-\alpha_{2 k}\left(y_{1}\right)}+e^{-\alpha_{2 k}\left(y_{2}\right)}\right) \sum_{m=0}^{2 k-1} C_{\alpha} \beta^{s\left(y_{1}, y_{2}\right)+1-m} \\
& \leq\left(e^{-\alpha_{2 k}\left(y_{1}\right)}+e^{-\alpha_{2 k}\left(y_{2}\right)}\right) C_{\alpha} \beta^{s\left(x_{1}, x_{2}\right)}(1-\beta)^{-1} .
\end{aligned}
$$

Applying the first item of Proposition 7.1 to the Banach space $\mathcal{B}_{0}$ and to the couples $(h, g)=\left(\tilde{\phi}^{(k)}, \tilde{\psi}^{(k)}\right)$ with $q_{1}>2$, we obtain that 


$$
\begin{aligned}
& \int_{\bar{M}} \phi^{(k)} 1_{\left\{\kappa_{n}=N\right\}} \psi^{(k)} \circ \bar{T}^{n} d \bar{\mu} \\
& \quad=\frac{1}{a_{n}^{d}}\left(I_{0}\left(\frac{N}{a_{n}}\right)-I_{2}\left(\frac{N}{a_{n}}\right) \frac{\log \log n}{2 \log n}\right) \int_{\bar{M}} \phi^{(k)} d \bar{\mu} \int_{\bar{M}} \psi^{(k)} d \bar{\mu}+O\left(\frac{\|\psi\|_{\infty}\|\phi\|_{\infty}}{\left(a_{n}\right)^{d} \log n}\right),
\end{aligned}
$$

where we used the fact that $\left|\psi^{(k)}\right| \leq|\psi|,\left|\phi^{(k)}\right| \leq|\phi|$ and $k / a_{n} \ll(\log n)^{-1}$. Moreover

$$
\left|\int_{\bar{M}} \phi d \bar{\mu} \int_{\bar{M}} \psi d \bar{\mu}-\int_{\bar{M}} \phi^{(k)} d \bar{\mu} \int_{\bar{M}} \psi^{(k)} d \bar{\mu}\right| \leq\|\psi\|_{\infty}\left\|\omega_{-k}^{k}(\phi, \cdot)\right\|_{L^{1}(\bar{\mu})}+\|\phi\|_{\infty}\left\|\omega_{-k}^{\infty}(\psi, \cdot)\right\|_{L^{1}(\bar{\mu})}
$$

and, setting $\phi^{(k,+)}(x):=\sup _{\xi_{-k}^{k}(x)}|\phi|$ and $\psi^{(k,+)}(x):=\sup _{\xi_{-k}^{\infty}(x)}|\psi|$,

$$
\begin{aligned}
& \int_{\bar{M}} 1_{\left\{\kappa_{n}=N\right\}}\left|\phi . \psi \circ \bar{T}^{n}-\phi^{(k)} \cdot \psi^{(k)} \circ \bar{T}^{n}\right| d \bar{\mu} \\
& \quad \leq \int_{\bar{M}} 1_{\left\{\kappa_{n}=N\right\}}\left(|\phi| \cdot\left|\psi-\psi^{(k)}\right| \circ \bar{T}^{n}+\left|\phi-\phi^{(k)}\right| \cdot\left|\psi^{(k)}\right| \circ \bar{T}^{n}\right) d \bar{\mu} \\
& \leq \int_{\bar{M}} 1_{\left\{\kappa_{n}=N\right\}}\left(\phi^{(k,+)} \cdot \omega_{-k}^{+\infty}(\psi, \cdot) \circ \bar{T}^{n}+\omega_{-k}^{k}(\phi, \cdot) \cdot \psi^{(k,+)} \circ \bar{T}^{n}\right) d \mu
\end{aligned}
$$

which is in

$$
O\left(I_{0}\left(\frac{N}{2 a_{n}}\right) a_{n}^{-d}\left(\left|\bar{\mu}\left(\phi^{(k,+)}\right)\right| \bar{\mu}\left(\omega_{-k}^{\infty}(\psi, \cdot)\right)+\bar{\mu}\left(\omega_{-k}^{k}(\phi, \cdot)\right)\left|\bar{\mu}\left(\psi^{(k,+)}\right)\right|\right)+\frac{\log \log n}{\left(a_{n}\right)^{d} \log n}\|\phi\|_{\infty}\|\psi\|_{\infty}\right)
$$

applying (111) with $\phi, \psi$ replaced respectively by $\left(\phi^{(k,+)}, \omega_{-k}^{\infty}(\psi)\right)$ and by $\left(\omega_{-k}^{k}(\phi), \psi^{(k,+)}\right)$, and using $\left|I_{0}(x)+I_{2}(x)\right| \ll I_{0}(x / 2)$. This ends the proof of $(106)$.

Assume now the assumptions of (b) and let us prove (107). We replace (108) by

$$
\phi^{(k)}(\bar{x}):=\mathbb{E}_{\bar{\mu}}\left[\phi \mid \xi_{-k}^{k}(x)\right] \text { and } \psi^{(k)}(\bar{x}):=\mathbb{E}_{\bar{\mu}}\left[\phi \mid \xi_{-k}^{k}(x)\right] .
$$

Observe that $\mathbb{E}_{\bar{\mu}}\left[\phi^{(k)}\right]=\mathbb{E}_{\bar{\mu}}[\phi], \mathbb{E}_{\bar{\mu}}\left[\psi^{(k)}\right]=\mathbb{E}_{\bar{\mu}}[\psi]$ and

$$
\mathbb{E}_{\mu_{\Delta}}\left[\hat{\kappa}_{k} P^{k} \tilde{\phi}^{(k)}\right]=\mathbb{E}_{\bar{\mu}}\left[\kappa_{k} \circ \bar{T}^{k} \phi^{(k)} \circ \bar{T}^{k}\right]=\mathbb{E}_{\bar{\mu}}\left[\kappa_{k} \phi^{(k)}\right]=\mathbb{E}_{\bar{\mu}}\left[\kappa_{k} \phi\right],
$$

due to (114) since $\kappa_{k}$ is $\xi_{-k}^{k}$-measurable and similarly, setting $\kappa_{-k}:=\sum_{m=-k}^{-1} \kappa \circ \bar{T}^{m}$, $\mathbb{E}_{\mu_{\Delta}}\left[\hat{\kappa}_{k} \tilde{\psi}^{(k)}\right]=\mathbb{E}_{\bar{\mu}}\left[\kappa_{k} \psi^{(k)} \circ \bar{T}^{k}\right]=\mathbb{E}_{\bar{\mu}}\left[\kappa_{k} \circ \bar{T}^{-k} \psi^{(k)}\right]=\mathbb{E}_{\bar{\mu}}\left[\kappa_{-k} \psi^{(k)}\right]=\mathbb{E}_{\bar{\mu}}\left[\kappa_{-k} \psi\right]$.

To prove (107), we apply the second item of Proposition 7.1 to the Banach space $\mathcal{B}_{0}$ and to the couples $(h, g)=\left(\tilde{\phi}^{(k)}, \tilde{\psi}^{(k)}\right)$ with $p<2$ (close to 2$), q_{0}>4$ (large), $p_{1}=\infty$, $q_{1}=1$ so that the condition $k(n) \leq C a_{n}^{\frac{\tilde{\gamma}}{1+\tilde{\gamma}}} /(\log n)^{1 /(1+\tilde{\gamma})}$ of Proposition 7.1 holds. We obtain that

$$
\begin{aligned}
\int_{\bar{M}} \phi^{(k)} 1_{\left\{\kappa_{n}=N\right\}} \psi^{(k)} \circ \bar{T}^{n} d \bar{\mu}=J_{1}+J_{2}+J_{3}+O\left(\frac{\left.\|\psi\|_{L^{q_{0}}(\bar{\mu})\|\phi\|_{\infty}}^{a_{n}^{d+1} \log n}\right)}{J_{1}}:=\frac{1}{a_{n}^{d}}\left(I_{0}\left(\frac{N}{a_{n}}\right)-\frac{1}{2} I_{2}\left(\frac{N}{a_{n}}\right) \frac{\log \log n}{\log n}+O\left(\frac{1}{\log n}\right)\right) \mathbb{E}_{\bar{\mu}}[\phi] \mathbb{E}_{\bar{\mu}}[\psi],\right. \\
J_{2}:=\frac{C_{k}\left(\tilde{\psi}^{(k)}, \tilde{\phi}^{(k)}\right)}{\left(a_{n}\right)^{d+1}}\left(I_{1}\left(\frac{N}{a_{n}}\right)-\frac{I_{3}\left(\frac{N}{a_{n}}\right)}{2} \frac{\log \log n}{\log n}\right)+O\left(a_{n}^{-d-1}(\log n)^{-1}\right),
\end{aligned}
$$




$$
J_{3}:=O\left(\frac{1}{(2 \pi)^{d}\left(a_{n}\right)^{d+1}} \int_{\left[-\beta a_{n}, \beta a_{n}\right]^{d}}|u| e^{-\frac{c_{0} \min \left(|u|^{2-\epsilon},|u|^{2+\epsilon)}\right.}{2}}\left|\mathbb{E}_{\mu_{\Delta}}\left[\tilde{\psi}^{(k)} \Pi_{0}^{\prime}\left(P_{u / a_{n}}^{k} P^{k} \tilde{\phi}^{(k)}-P^{2 k} \tilde{\phi}^{(k)}\right)\right]\right| d u\right) .
$$

Note that $\phi=\phi_{0}+\bar{\mu}(\phi)$ and $\psi=\psi_{0}+\bar{\mu}(\psi)$, with $\phi_{0}=\phi-\bar{\mu}(\phi)$ and $\psi_{0}=\psi-\bar{\mu}(\psi)$. We can resume the rest of the proof to estimating $J_{2}$ and $J_{3}$. To study these two terms, we shall exploit the expression $\Pi_{0}^{\prime}$ obtained in Propositions 5.3 and 5.4. For $J_{3}$ we exploit that

$$
\Pi_{0}^{\prime} w=\mathbb{E}_{\bar{\mu}}[w] H+i \sum_{j \geq 0} \mathbb{E}_{\mu_{\Delta}}\left[\hat{\kappa} \circ f^{j} w\right] 1_{\Delta}
$$

with $H(x):=Q_{0}^{\prime}\left(1_{Y}\right) \circ \pi_{0}(x)+i \sum_{m=0}^{\omega(x)-1} \hat{\kappa} \circ f^{m} \circ \pi_{0}(x)+\frac{i}{\mu_{\Delta}(Y)} \sum_{j \geq 0} \mathbb{E}_{\mu_{\Delta}}\left[\hat{\kappa} 1_{Y} \circ f^{j+1}\right]$.

Writing $t$ for $u / a_{n}$ in the expression of $J_{3}$ and using the above formula for $\Pi_{0}^{\prime}$ we note that

$$
\begin{aligned}
& \left|\mathbb{E}_{\mu_{\Delta}}\left[\tilde{\psi}^{(k)} \Pi_{0}^{\prime}\left(P_{t}^{k} P^{k}-P^{2 k}\right) \tilde{\phi}^{(k)}\right]\right| \\
& \quad=\left|\mathbb{E}_{\bar{\mu}}\left[\left(e^{i t \kappa_{k}}-1\right) \phi^{(k)}\right] \mathbb{E}_{\mu_{\Delta}}\left[\tilde{\psi}^{(k)} H\right]+i \mathbb{E}_{\bar{\mu}}\left[\psi^{(k)}\right] \sum_{j \geq 0} \mathbb{E}_{\mu_{\Delta}}\left[\hat{\kappa} P^{j}\left(P_{t}^{k} P^{k}-P^{2 k}\right) \tilde{\phi}^{(k)}\right]\right| \\
& \quad \leq|t| k\|\kappa\|_{L^{1}(\bar{\mu})}\|\phi\|_{\infty}\|\psi\|_{\infty}\|H\|_{L^{1}(\bar{\mu})}+\|\psi\|_{\infty} E_{k}, \\
& E_{k}=\sum_{j=0}^{\log n-1}\left|\mathbb{E}_{\bar{\mu}}\left[\kappa \circ \bar{T}^{k+j}\left(e^{i t \kappa_{k}}-1\right) \phi^{(k)}\right]\right|+\sum_{j \geq \log n}\|\hat{\kappa}\|_{L^{q 1}} \theta^{j}\left\|\left(P_{t}^{k} P^{k}-P^{2 k}\right) \tilde{\phi}^{(k)}\right\|_{\mathcal{B}_{0}} \\
& \quad \ll \sum_{j=0}^{\log n-1}\left\|\kappa \circ \bar{T}^{k+j}\left(t \kappa_{k}\right)^{p-1}\right\|_{L^{1}(\bar{\mu})}\|\phi\|_{\infty}+\sum_{j \geq \log n} \theta^{j}\|\phi\|_{\infty},
\end{aligned}
$$

$E_{k} \ll \log n\|\kappa\|_{L^{p}\left(\mu_{\Delta}\right)}\left\|t \kappa_{k}\right\|_{L^{p}(\bar{\mu})}^{p-1}\|\phi\|_{\infty}+\theta^{\log n}\|\phi\|_{\infty} \ll\left(|t k|^{p-1} \log n+\theta^{\log n}\right)\|\phi\|_{\infty}$.

This together with equation (117) and $k(n) \ll a_{n}^{\frac{1}{2}-u}$ implies that $J_{3}=O\left(a_{n}^{-d-1} / \log n\right)$. The rest of the proof is allocated to the study of $J_{2}$. We will use

$$
\mathbb{E}_{\mu_{\Delta}}\left[\tilde{\psi}^{(k)} \Pi_{0}^{\prime} P^{2 k} \tilde{\phi}^{(k)}\right]=\mathbb{E}_{\bar{\mu}}\left[\phi^{(k)}\right] \mathbb{E}_{\mu_{\Delta}}\left[\tilde{\psi}^{(k)} H\right]+i \mathbb{E}_{\bar{\mu}}\left[\psi^{(k)}\right] \sum_{j \geq 0} \mathbb{E}_{\bar{\mu}}\left[\kappa \circ \bar{T}^{k+j} \phi^{(k)}\right],
$$

obtained from (116) with $w=\tilde{\phi}^{(k)}$, after multiplication with $\tilde{\psi}^{(k)}$ and integration. Thus

$$
J_{2}=\frac{\mathfrak{C}_{k(n)}(\phi, \psi)}{\left(a_{n}\right)^{d+1}}\left(I_{1}\left(\frac{N}{a_{n}}\right)-\frac{I_{3}\left(\frac{N}{a_{n}}\right)}{2} \frac{\log \log n}{\log n}\right)+O\left(a_{n}^{-d-1} / \log n\right),
$$

with $\mathfrak{C}_{k}(\phi, \psi):=C_{k}\left(\tilde{\psi}^{(k)}, \tilde{\phi}^{(k)}\right)$, that is

$$
\mathfrak{C}_{k}(\phi, \psi)=\mathbb{E}_{\mu_{\Delta}}\left[\tilde{\psi}^{(k)} \Pi_{0}^{\prime} P^{2 k} \tilde{\phi}^{(k)}\right]+i \mathbb{E}_{\bar{\mu}}\left[\kappa_{-k} \psi^{(k)}\right] \mathbb{E}_{\mu_{\Delta}}\left[\phi^{(k)}\right]+i \mathbb{E}_{\bar{\mu}}\left[\psi^{(k)}\right] \mathbb{E}_{\bar{\mu}}\left[\kappa_{k} \phi^{(k)}\right]
$$




$$
=\mathbb{E}_{\bar{\mu}}[\phi]\left(\mathbb{E}_{\mu_{\Delta}}\left[\tilde{\psi}^{(k)} H\right]+i \mathbb{E}_{\bar{\mu}}\left[\kappa_{-k} \psi^{(k)}\right]\right)+i \mathbb{E}_{\bar{\mu}}[\psi] \sum_{j \geq 0} \mathbb{E}_{\bar{\mu}}\left[\kappa \circ \bar{T}^{j} \phi^{(k)}\right] .
$$

Thus

$$
\begin{aligned}
& \int_{\bar{M}} \phi^{(k)} 1_{\left\{\kappa_{n}=N\right\}} \psi^{(k)} \circ \bar{T}^{n} d \bar{\mu} \\
& =\frac{1}{a_{n}^{d}}\left(I_{0}\left(\frac{N}{a_{n}}\right)-\frac{1}{2} I_{2}\left(\frac{N}{a_{n}}\right) \frac{\log \log n}{\log n}+O\left(\frac{1}{\log n}\right)\right) \mathbb{E}_{\bar{\mu}}[\phi] \mathbb{E}_{\bar{\mu}}[\psi] \\
& +\frac{\mathfrak{C}_{k(n)}(\phi, \psi)}{\left(a_{n}\right)^{d+1}}\left(I_{1}\left(\frac{N}{a_{n}}\right)-\frac{I_{3}\left(\frac{N}{a_{n}}\right)}{2} \frac{\log \log n}{\log n}\right)+O\left(a_{n}^{-d-1} / \log n\right) .
\end{aligned}
$$

In particular, since $\left|\psi^{(k)}\right| \leq \psi^{(k,+)} \leq|\psi|+\omega_{-k}^{k}(\psi, \cdot)$ with $\psi^{(k,+)}(x):=\sum_{y \in \xi_{-k}^{k}(x)}|\psi(y)|$,

$$
\begin{aligned}
& \left|\int_{\bar{M}} 1_{\left\{\kappa_{n}=N\right\}}\left(\phi \cdot \psi \circ \bar{T}^{n}-\phi^{(k)} \cdot \psi^{(k)} \circ \bar{T}^{n}\right) d \bar{\mu}\right| \\
& \quad \leq \int_{\bar{M}} 1_{\left\{\kappa_{n}=N\right\}}\left(\left|\phi \cdot \omega_{-k}^{k}(\psi, \cdot) \circ \bar{T}^{n}\right|+\left|\omega_{-k}^{k}(\phi, \cdot) \cdot \psi^{(k,+)} \circ \bar{T}^{n}\right|\right) d \bar{\mu}
\end{aligned}
$$

which is dominated, up to a multiplicative constant by

$$
\begin{aligned}
\ll & \frac{\|\phi\|_{\infty}\left\|\omega_{-k}^{k}(\psi, \cdot)\right\|_{L^{1}(\bar{\mu})}+\left\|\psi^{(k,+)}\right\|_{\infty}\left\|\omega_{-k}^{k}(\phi, \cdot)\right\|_{L^{1}(\bar{\mu})}}{a_{n}^{d}} \\
& +\frac{\left.\mathfrak{C}_{k(n)}\left(|\phi|, \omega_{-k}^{k}(\psi, \cdot)\right)+\mathfrak{C}_{k(n)}\left(\omega_{-k}^{k}(\phi, \cdot),\left|\psi^{(k,+)}\right|\right)\right)}{\left(a_{n}\right)^{d+1}}+O\left(a_{n}^{-d-1} / \log n\right) \\
= & O\left(a_{n}^{-d-1} / \log n\right),
\end{aligned}
$$

since for $(h, g)=\left(|\phi|, \omega_{-k}^{k}(\psi, \cdot)\right)$ or $(h, g)=\left(\omega_{-k}^{k}(\phi, \cdot), \psi^{(k,+)}\right)$,

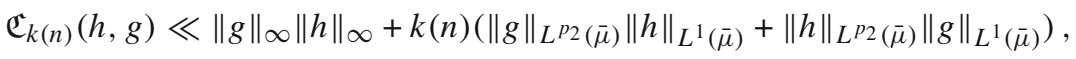

(since $\left.\kappa \in L^{p_{2} /\left(p_{2}-1\right)(\bar{\mu})}\right)$ and using our assumptions. Therefore

$$
\begin{aligned}
\int_{\bar{M}} \phi 1_{\left\{\kappa_{n}=N\right\}} \psi \circ \bar{T}^{n} d \bar{\mu} \\
=\frac{1}{a_{n}^{d}}\left(I_{0}\left(\frac{N}{a_{n}}\right)-\frac{1}{2} I_{2}\left(\frac{N}{a_{n}}\right) \frac{\log \log n}{\log n}+O\left(\frac{1}{\log n}\right)\right) \int_{\bar{M}} \phi d \bar{\mu} \int_{\bar{M}} \psi d \bar{\mu} \\
\quad+\frac{\mathfrak{C}_{k(n)}(\phi, \psi)}{\left(a_{n}\right)^{d+1}}\left(I_{1}\left(\frac{N}{a_{n}}\right)-\frac{I_{3}\left(\frac{N}{a_{n}}\right)}{2} \frac{\log \log n}{\log n}\right)+O\left(a_{n}^{-d-1} / \log n\right) .
\end{aligned}
$$

Note that $\phi=\phi_{0}+\bar{\mu}(\phi)$ and $\psi=\psi_{0}+\bar{\mu}(\psi)$, with $\phi_{0}=\phi-\bar{\mu}(\phi)$ and $\psi_{0}=\psi-\bar{\mu}(\psi)$. So

$$
\int_{\bar{M}} \phi 1_{\left\{\kappa_{n}=N\right\}} \psi \circ \bar{T}^{n} d \bar{\mu}
$$




$$
=\int_{\bar{M}} \phi_{0} 1_{\left\{\kappa_{n}=N\right\}} \psi \circ \bar{T}^{n} d \bar{\mu}+\bar{\mu}(\phi) \int_{\bar{M}} 1_{\left\{\kappa_{n}=N\right\}} \psi_{0} \circ \bar{T}^{n} d \bar{\mu}+\bar{\mu}(\phi) \bar{\mu}(\psi) \bar{\mu}\left(\kappa_{n}=N\right) .
$$

Applying (121) with $\left(1_{\bar{M}}, 1_{\bar{M}}\right)$ instead of $(\phi, \psi)$ leads to

$$
\bar{\mu}\left(\kappa_{n}=N\right)=\frac{1}{a_{n}^{d}}\left(I_{0}\left(\frac{N}{a_{n}}\right)-\frac{1}{2} I_{2}\left(\frac{N}{a_{n}}\right) \frac{\log \log n}{\log n}+O\left(\frac{1}{\log n}\right)\right)+O\left(a_{n}^{-d-1} / \log n\right),
$$

since $\mathbb{E}_{\mu_{\Delta}}\left[\Pi_{0}^{\prime} 1\right]=0$ (see Remark 5.13). Now let us study $\int_{\bar{M}} \phi_{0} 1_{\left\{\kappa_{n}=N\right\}} \psi \circ \bar{T}^{n} d \bar{\mu}$. In what follows we show that $\mathfrak{C}_{k(n)}\left(\phi_{0}, \psi\right)$ converges as $n \rightarrow+\infty$ with rate in $O\left((\log n)^{-1}\right)$. Note that

$$
\mathfrak{C}_{k(n)}\left(\phi_{0}, \psi\right)=i \mathbb{E}_{\bar{\mu}}[\psi] \sum_{j \geq 0} \mathbb{E}_{\bar{\mu}}\left[\kappa \circ \bar{T}^{j} \phi^{(k(n))}\right] .
$$

Let us prove the two following estimates

$$
\begin{aligned}
& \left|\sum_{j \geq 0} \mathbb{E}_{\bar{\mu}}\left[\kappa \circ \bar{T}^{j} \phi^{(k(n))}\right]-\sum_{j \geq 0} \mathbb{E}_{\bar{\mu}}\left[\kappa \circ \bar{T}^{j} \phi\right]\right|=O\left((\log n)^{-1}\right), \\
& \sum_{j \geq 0} \mid \mathbb{E}_{\bar{\mu}\left[\kappa \circ \bar{T}^{j} \phi\right] \mid<\infty .} .
\end{aligned}
$$

- Proof of (126). First, for every integers $j, k_{j} \geq 0$, we have

$$
\begin{aligned}
& \left|\mathbb{E}_{\bar{\mu}}\left[\kappa \circ \bar{T}^{j} \phi\right]\right| \leq\left|\mathbb{E}_{\bar{\mu}}\left[\kappa \circ \bar{T}^{j} \phi^{\left(k_{j}\right)}\right]\right|+\left|\mathbb{E}_{\bar{\mu}}\left[\kappa \circ \bar{T}^{j}\left(\phi-\phi^{\left(k_{j}\right)}\right)\right]\right| \\
& \leq \mid \mathbb{E}_{\mu_{\Delta}}\left[\hat{\kappa} P^{j+k_{j}}\left(\tilde{\phi}^{\left(k_{j}\right)}-\mu_{\Delta}\left(\tilde{\phi}^{\left(k_{j}\right)}\right)\right] \mid+\|\kappa\|_{L^{\frac{p_{2}}{p^{-1}}(\bar{\mu})}}\left\|\omega_{-k_{j}}^{k_{j}}(\phi, \cdot)\right\|_{L^{p_{2}(\bar{\mu})}} .\right.
\end{aligned}
$$

Now using the fact that $\left\|P^{2 k_{j}} \tilde{\phi}^{\left(k_{j}\right)}-\mu_{\Delta}\left(\tilde{\phi}^{\left(k_{j}\right)}\right)\right\|_{\mathcal{B}_{0}} \ll\|\phi\|_{\infty}$ (which comes from (110)) and using (16) for $t=0$, we conclude that

$$
\left|\mathbb{E}_{\bar{\mu}}\left[\kappa \circ \bar{T}^{j} \phi\right]\right| \leq C\|\hat{\kappa}\|_{L^{q_{1}}\left(\mu_{\Delta}\right)} \theta^{j-k_{j}}\|\phi\|_{\infty}+\|\kappa\|_{L^{\frac{p_{2}}{p_{2}-1}(\bar{\mu})}}\left\|\omega_{-k_{j}}^{k_{j}}(\phi, \cdot)\right\|_{L^{p_{2}}(\bar{\mu})} .
$$

Taking $k_{j}=j / 2$, we obtain

$$
\sum_{j \geq 0}\left|\mathbb{E}_{\bar{\mu}}\left[\kappa \circ \bar{T}^{j} \phi\right]\right| \ll \sum_{j \geq 0}\left(\|\hat{\kappa}\|_{L^{\frac{p}{p-1}}} \theta^{j / 2}\|\phi\|_{\infty}+\left\|\omega_{-\lfloor j / 2\rfloor}^{L j / 2\rfloor}(\phi, \cdot)\right\|_{L^{p_{2}}(\bar{\mu})}\right)<\infty .
$$

This ends the proof of (126).

- Proof of (125). Recall that $\left\|\mathbb{E}_{\bar{\mu}}\left[\psi^{(k)}-\psi\right]\right\| \leq\left\|\omega_{-k}^{k}(\psi, \cdot)\right\|_{L^{1}(\bar{\mu})}=O\left((\log n)^{-1}\right)$.

Observe that, due to (127) with $k_{j}:=\lfloor j / 2\rfloor$ and $k=k(n)$,

$$
\begin{aligned}
& \sum_{j \geq 0}\left|\mathbb{E}_{\bar{\mu}}\left[\kappa \circ \bar{T}^{j}\left(\phi^{(k)}-\phi\right)\right]\right|=\sum_{j=0}^{2 k-1}\left|\mathbb{E}_{\bar{\mu}}\left[\kappa \circ \bar{T}^{j}\left(\phi^{(k)}-\phi\right)\right]\right|+\sum_{j \geq 2 k}\left|\mathbb{E}_{\bar{\mu}}\left[\kappa \circ \bar{T}^{j}\left(\phi^{(k)}-\phi\right)\right]\right| \\
& \leq 2 k\|\kappa\|_{L^{\frac{p_{2}}{p_{2}-1}}(\bar{\mu})}\left\|\omega_{-k}^{k}(\phi, \cdot)\right\|_{L^{p_{2}}(\bar{\mu})}
\end{aligned}
$$

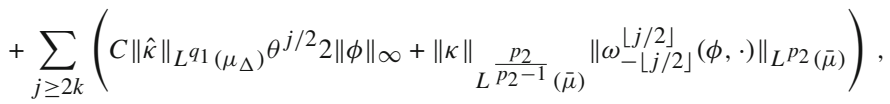

which is $O\left((\log n)^{-1}\right)$ due to our assumptions. This ends the proof of (125). 
Applying (124) combined with (125) and (126), we obtain

$$
\mathfrak{C}_{k}\left(\phi_{0}, \psi\right)=i \mathbb{E}_{\bar{\mu}}[\psi] \sum_{j \geq 0} \mathbb{E}_{\bar{\mu}}\left[\kappa \circ \bar{T}^{j} \phi\right]+O\left((\log n)^{-1}\right)
$$

and thus, due to (121),

$$
\int_{\bar{M}} \phi_{0} 1_{\left\{\kappa_{n}=N\right\}} \psi \circ \bar{T}^{n} d \bar{\mu}=\frac{i \mathbb{E}_{\bar{\mu}}[\psi] \sum_{j \geq 0} \mathbb{E}_{\bar{\mu}}\left[\kappa \circ \bar{T}^{j} \phi\right]}{\left(a_{n}\right)^{d+1}} \tilde{I}_{1}\left(n, \frac{N}{a_{n}}\right)+O\left(\frac{a_{n}^{-d-1}}{\log n}\right),
$$

with $\tilde{I}_{1}(n, x):=I_{1}(x)-\frac{I_{3}(x)}{2} \frac{\log \log n}{\log n}$. Now let us prove that a similar formula holds for $\left(1, \psi_{0}\right)$, where $\psi_{0}=\psi-\bar{\mu}(\psi)$ thanks to time reversibility. Let $\tau: \bar{M} \rightarrow \bar{M}$ be given by $\tau(q, \vec{v})=\left(q, 2\left(\vec{n}_{q}, \vec{v}\right) \vec{n}_{q}-\vec{v}\right)$ where $\vec{n}_{q}$ is the unit normal vector to $\partial Q$ directed in $Q$. Observe that $\tau$ preserves $\bar{\mu}$ and satisfies the following relations: $\tau \circ \tau=i d$, $\tau \circ \bar{T}^{n} \circ \tau=\bar{T}^{-n}$ and $\kappa \circ \bar{T}^{k} \circ \tau:=-\kappa \circ \bar{T}^{-k-1}$. In particular, $\kappa_{n} \circ \tau \circ \bar{T}^{n}=-\kappa_{n}$. Therefore

$$
\begin{aligned}
& \int_{\bar{M}} 1_{\left\{\kappa_{n}=N\right\}} \psi_{0} \circ \bar{T}^{n} d \bar{\mu}=\int_{\bar{M}} 1_{\left\{\kappa_{n} \circ \tau=N\right\}} \psi_{0} \circ \bar{T}^{n} \circ \tau d \bar{\mu} \\
& =\int_{\bar{M}} \tau 1_{\left\{\kappa_{n} \circ \tau=N\right\}} \psi_{0} \circ \tau \circ \bar{T}^{-n} d \bar{\mu}=\int_{\bar{M}} 1_{\left\{\kappa_{n} \circ \tau \circ \bar{T}^{n}=N\right\}} \psi_{0} \circ \tau d \bar{\mu} \\
& =\int_{\bar{M}} \psi_{0} \circ \tau 1_{\left\{\kappa_{n}=-N\right\}} d \bar{\mu} .
\end{aligned}
$$

Observe that $\omega_{-k}^{k}\left(\psi_{0} \circ \tau, x\right)=\omega_{-k}^{k}\left(\psi_{0}, \tau(x)\right)$ and so, the composition by $\tau$ preserves the $L^{r}$ norms of $\omega_{-k}^{k}$. Thus, applying (130) to the couple $\left(\psi_{0} \circ \tau, 1\right)$ instead of $\left(\phi_{0}, \psi\right)$, we obtain

$$
\begin{aligned}
& \int_{\bar{M}} 1_{\left\{\kappa_{n}=N\right\}} \psi_{0} \circ \bar{T}^{n} d \bar{\mu}=\frac{i \sum_{j \geq 0} \mathbb{E}_{\bar{\mu}}\left[\kappa \circ \bar{T}^{j} \psi \circ \tau\right]}{\left(a_{n}\right)^{d+1}} \tilde{I}_{1}\left(n,-\frac{N}{a_{n}}\right)+O\left(\frac{a_{n}^{-d-1}}{\log n}\right) \\
& =\frac{i \sum_{j \leq-1} \mathbb{E}_{\bar{\mu}}\left[\kappa \circ \bar{T}^{j} \psi\right]}{\left(a_{n}\right)^{d+1}} \tilde{I}_{1}\left(n, \frac{N}{a_{n}}\right)+O\left(\frac{a_{n}^{-d-1}}{\log n}\right) .
\end{aligned}
$$

since $\mathbb{E}_{\bar{\mu}}\left[\kappa \circ \bar{T}^{j} \psi \circ \tau\right]=\mathbb{E}_{\bar{\mu}}\left[-\kappa \circ \bar{T}^{-j-1} \psi\right],-\tilde{I}_{1}(n,-x)=\tilde{I}_{1}(n, x)$. The claimed estimate follows from this last estimate combined with (122), (123) and (130).

For all $N \in \mathbb{Z}^{d}$, we set $\phi_{N}:=\phi 1_{M_{N}}$. Observe that $\omega_{-k}^{\infty}(h, \cdot) \leq \omega_{-k}^{k}(h, \cdot) \leq L_{h, \eta} \eta^{k}$. Therefore, Theorem 2.2 (resp. Theorem 2.4) is a direct consequence of the previous (resp. following) theorem (for Theorem 2.4 we also use the remark just after its statement).

Theorem 7.7. Let $(k(n))_{n}$ be a sequence of integers diverging to $+\infty$ such that $k(n)=$ $O\left(a_{n} / \log n\right)$. Let $\phi, \psi: M \rightarrow \mathbb{C}$ be two measurable functions.

(I) If $\sum_{N \in \mathbb{Z}^{d}}\left(\left\|\phi_{N}\right\|_{\infty}+\left\|\psi_{N}\right\|_{\infty}\right)<\infty$ and $\lim _{k \rightarrow+\infty} \int_{M}\left(\omega_{-k}^{k}(\phi, \cdot)+\omega_{-k}^{\infty}(\psi, \cdot)\right) d \mu=$ 0. Then

$$
\int_{M} \phi . \psi \circ T^{n} d \mu=\frac{I_{0}(0)}{a_{n}^{d}} \int_{M} \phi d \mu \int_{M} \psi d \mu+o\left(a_{n}^{-d}\right) .
$$


(II) If moreover there exists $\gamma \in(0,1)$ such that $\sum_{N}|N|^{\gamma}\left(\left\|\phi_{N}\right\|_{\infty}+\left\|\psi_{N}\right\|_{\infty}\right)<\infty$, and if $\int_{M}\left(\omega_{-k(n)}^{k(n)}(\phi, \cdot)+\omega_{-k(n)}^{\infty}(\psi, \cdot)\right) d \mu=O\left((\log n)^{-1}\right)$, then

$$
\int_{M} \phi \cdot \psi \circ T^{n} d \mu=\frac{I_{0}(0)}{a_{n}^{d}}\left(1-\frac{d \log \log n}{2 \log n}\right) \int_{M} \phi d \mu \int_{M} \psi d \mu+O\left(\frac{1}{a_{n}^{d} \log n}\right) .
$$

(III) If moreover $k(n) \ll a_{n}^{\frac{1}{2}-u}$ for some $u \in(0,1)$ and if there exists $\gamma \in(0,1)$ such that $\sum_{N}|N|^{1+\gamma}\left(\left\|\phi_{N}\right\|_{\infty}+\left\|\psi_{N}\right\|_{\infty}\right)<\infty$, and $\int_{M}\left(\omega_{-k(n)}^{k(n)}(\phi, \cdot)+\omega_{-k(n)}^{k(n)}(\psi, \cdot)\right) d \mu=$ $O\left(\left(a_{n} \log n\right)^{-1}\right)$, then

$$
\int_{M} \phi \cdot \psi \circ T^{n} d \mu=\frac{I_{0}(0)}{a_{n}^{d}}\left(1-\frac{d \log \log n+O(1)}{2 \log n}\right) \int_{M} \phi d \mu \int_{M} \psi d \mu+O\left(\frac{1}{a_{n}^{d+1} \log n}\right) .
$$

Proof. For every $x \in M$ we write $\bar{x} \in \bar{M}$ for the class of $x$ modulo $\mathbb{Z}^{d}$ for the position. Let us write $\bar{\phi}_{N}(\bar{x})=\phi_{N}(x)$, for every $x \in M_{N}$. Observe that

$$
\int_{M} \phi . \psi \circ T^{n} d \mu=\sum_{N_{1}, N_{2} \in \mathbb{Z}^{2}} \int_{\bar{M}} \bar{\phi}_{N_{1}} \mathbf{1}_{\left\{\kappa_{n}=N_{2}-N_{1}\right\}} \bar{\psi}_{N_{2}} \circ \bar{T}^{n} d \bar{\mu} .
$$

Now, setting again $\tilde{I}_{0}(n, x):=I_{0}(x)-\frac{I_{2}(x)}{2} \frac{\log \log n}{\log n}$ and applying (106), we obtain

$$
\begin{aligned}
& \int_{M} \phi . \psi \circ T^{n} d \mu=\sum_{N_{1}, N_{2} \in \mathbb{Z}^{d}} \frac{1}{a_{n}^{d}} \tilde{I}_{0}\left(n, \frac{N_{2}-N_{1}}{a_{n}}\right) \int_{M} \phi_{N_{1}} d \mu \int_{M} \psi_{N_{2}} d \mu \\
& \left.+O\left(a_{n}^{-d} \sum_{N_{1}, N_{2} \in \mathbb{Z}^{d}} I_{0}\left(\frac{N_{2}-N_{1}}{2 a_{n}}\right)\left\|\phi_{N_{1}}\right\|_{\infty}\left\|\omega_{-k(n)}^{\infty}\left(\psi_{N_{2}}, \cdot\right)\right\|_{L^{1}(\bar{\mu})}\right)\right) \\
& \left.+O\left(a_{n}^{-d} \sum_{N_{1}, N_{2} \in \mathbb{Z}^{d}} I_{0}\left(\frac{N_{2}-N_{1}}{2 a_{n}}\right)\left\|\psi_{N_{2}}\right\| \infty\left\|\omega_{-k(n)}^{k(n)}\left(\phi_{N_{1}}, \cdot\right)\right\|_{L^{1}(\bar{\mu})}\right)\right)+O\left(\frac{\left\|\phi_{N_{1}}\right\| \infty\left\|\psi_{N_{2}}\right\|_{\infty}}{a_{n}^{d} \log n}\right) .
\end{aligned}
$$

(131) follows from (133) and the Lebesgue dominated convergence theorem, since $I_{0}$ and $I_{2}$ are bounded and since $I_{0}$ is continuous at 0 .

Assume now the assumptions of (II). Since $\left|I_{0}(X)-I_{0}(0)\right|+\left|I_{2}(X)-I_{2}(0)\right|=$ $O\left(X^{\gamma}\right)$, replacing $I_{0}\left(\frac{N_{2}-N_{1}}{a_{n}}\right)-\frac{1}{2} I_{2}\left(\frac{N_{2}-N_{1}}{a_{n}}\right) \ldots$ by $I_{0}(0)-\frac{1}{2} I_{2}(0) \ldots$ in (133) leads to an error term in $O\left(a_{n}^{-d-\gamma}\right)$. Since $I_{0}$ is bounded and to our assumptions, the first error term in (133) is in $O\left(a_{n}^{-d} / \log n\right)$. This completes the proof of (132).

Finally, we assume the assumptions of (III). We start from (115) for $\left(\bar{\phi}_{N_{1}}, \bar{\psi}_{N_{2}}, N_{2}-\right.$ $\left.N_{1}\right)$ instead of $(\phi, \psi, N)$. Our assumptions combined with $\left|I_{2 j}(x)-I_{2 j}(0)\right| \ll x^{1+\gamma}$ ensure that we can replace $I_{0}\left(N / a_{n}\right)$ and $I_{2}\left(N / a_{n}\right)$ in $J_{1}$ up to an error (after summation over $\left.N_{1}, N_{2} \in \mathbb{Z}^{2}\right)$ in $O\left(a_{n}^{-d-1-\gamma}\right)$. Our assumptions ensure that we can replace $\phi^{(k)}$ and $\psi^{(k)}$ by respectively $\phi$ and $\psi$ in $J_{1}$ up to a total error (after summation) in $O\left(a_{n}^{-d-1} / \log n\right)$. The fact that $\left|I_{2 j+1}(x)\right| \ll x^{\gamma}$, combined with our conditions, implies that the contribution (after summation) of $J_{2}$ is in $O\left(a_{n}^{-d-1} / \log n\right)$. We prove as in the previous result that the contribution (after summation) of $J_{3}$ is in $O\left(a_{n}^{-d-1} / \log n\right)$. We conclude using $I_{2}(0)=d I_{0}(0)$. 
Proof of Theorem 2.5. We proceed as in the proof of Proposition 7.4 with same notation $I_{\ell_{1}, \ell_{2}, N}$ introduced just before Proposition 7.4 with $A=\Sigma^{2}$ and

$$
J_{\ell_{1}, \ell_{2}, n, N, N_{0}}:=\int_{-\left[\beta a_{n}, \beta a_{n}\right]^{d}} e^{-i u \cdot N / a_{n}}\left(-i u \cdot N_{0}\right)^{\ell_{1}}(-A u \cdot u)^{\ell_{2}} \lambda_{u / a_{n}}^{n-2 k} d u
$$

Using again (98) and (99), we observe that

$$
\begin{aligned}
& \forall \delta_{0} \in(0,1], \quad:=J_{\ell_{1}, \ell_{2}, n, 0, N_{0}}+O\left(\frac{N_{0}^{\ell_{1}} N^{\delta_{0}}}{a_{n}^{\delta_{0}}}\right), \\
& J_{\ell_{1}, \ell_{2}, n, 0, N_{0}}=I_{\ell_{1}, \ell_{2}, N_{0}}(0)+I_{\ell_{1}, \ell_{2}+1, N_{0}}(0) \frac{\log \log n}{2 \log n}+O\left(\frac{N_{0}^{\ell_{1}}}{\log n}\right) .
\end{aligned}
$$

For every $N \in \mathbb{Z}^{d}$, we consider the functions $h_{N}, g_{N}: \bar{M} \rightarrow \mathbb{C}$ such that, for any $x \in M_{N}, h_{N}(\bar{x}):=\phi_{N}(x)$ and $g_{N}(\bar{x}):=\psi_{N}(x)$ where $\bar{x} \in \bar{M}$ is the class of $x$ modulo $\mathbb{Z}^{2}$ for the position. We take $k=k(n):=\lfloor(d+m)(\log n) /|\log \eta|\rfloor$ with $m:=3 / 2$ in the setting of the second item (which implies that $\eta^{k(n)} \ll a_{n}^{-d-2 m}$ ) and define the approximating functions $\phi^{(k)}, \psi^{(k)}: M \rightarrow \mathbb{R}$ ang $h_{N}^{(k)}, g_{N}^{(k)}: \bar{M} \rightarrow \mathbb{C}$ given by $\phi^{(k)}(x):=$ $\inf _{\xi_{-k}^{k}(x)} \phi_{+}-\sup _{\xi_{-k}^{k}(x)} \phi_{-}, \psi^{(k)}(x):=\inf _{\xi_{-k}^{\infty}(x)} \psi_{+}-\sup _{\xi_{-k}^{\infty}(x)} \psi_{-}, h_{N}^{(k)}(\bar{x}):=$ $\inf _{\xi_{-k}^{k}(\bar{x})}\left(h_{N}\right)_{+}-\sup _{\xi_{-k}^{k}(\bar{x})}\left(h_{N}\right)_{-} g_{N}^{(k)}(\bar{x}):=\inf _{\xi_{-k}^{k}(\bar{x})}\left(g_{N}\right)_{+}-\sup _{\xi_{-k}^{k}(\bar{x})}\left(g_{N}\right)_{-}$. Due to our choice of $k$ and to our assumptions on $\phi, \psi$,

$$
\int_{M} \phi \cdot \psi \circ(i d-T)^{m} \circ T^{n} d \mu=\int_{M} \phi^{(k)} \cdot \psi^{(k)} \circ(i d-T)^{m} \circ T^{n} d \mu+O\left(\eta^{k(n)}\right) \text {. }
$$

The error term in the previous formula is in $O\left(a_{n}^{-d-2 m}\right)$. So we focus on the following integral

$$
\int_{M} \phi^{(k)} \cdot \psi^{(k)} \circ T^{n} d \mu=\sum_{N_{1}, N_{2} \in \mathbb{Z}^{d}} \mathbb{E}_{\bar{\mu}}\left[h_{N_{1}}^{(k)} 1_{\left\{\kappa=N_{2}-N_{1}\right\}} g_{N_{2}}^{(k)} \circ \bar{T}^{n}\right]
$$

Recall, from the proof of Theorem 7.6 that, due the definition of $h_{N}^{(k)}, g_{N}^{(k)}$ there exist $\tilde{h}_{N}^{(k)}, \tilde{g}_{N}^{(k)}: \Delta \rightarrow \mathbb{R}$ such that $h_{N}^{(k)} \circ T^{k} \circ \mathfrak{p}_{1}=\tilde{h}_{N}^{(k)} \circ \mathfrak{p}_{2}, g_{N}^{(k)} \circ T^{k} \circ \mathfrak{p}_{1}=\tilde{g}_{N}^{(k)} \circ \mathfrak{p}_{2}$,

$$
\left\|\tilde{g}_{N}^{(k)}\right\|_{\infty} \leq\left\|g_{N}\right\|_{\infty} \text { and } \sup _{t}\left\|P_{t}^{k} P_{t} \tilde{h}_{N}^{(k)}\right\|_{\mathcal{B}_{0}} \leq\left\|h_{N}\right\|_{\infty},
$$

and thus, as seen at the begining of the proof of Proposition 7.1,

$$
\begin{aligned}
\int_{M} \phi^{(k)} \cdot \psi^{(k)} \circ T^{n} d \mu & =\sum_{N_{1}, N_{2} \in \mathbb{Z}^{d}} \mathbb{E}_{\mu_{\Delta}}\left[\tilde{h}_{N_{1}}^{(k)} 1_{\left\{\hat{\kappa}_{n} \circ f^{k}=N_{2}-N_{1}\right\}} \tilde{g}_{N_{2}}^{(k)} \circ \bar{T}^{n}\right] \\
& =\sum_{N_{1}, N_{2} \in \mathbb{Z}^{d}} \frac{1}{(2 \pi)^{d}} \int_{[-\pi, \pi]^{d}} e^{-i t \cdot\left(N_{2}-N_{1}\right)} \mathbb{E}_{\mu_{\Delta}}\left[P_{t}^{k}\left(\tilde{g}_{N_{2}}^{(k)} P_{t}^{n-2 k} P_{t}^{k} P^{k} \tilde{h}_{N_{1}}^{(k)}\right)\right] .
\end{aligned}
$$


- Let us assume the assumptions of item (a) of the theorem. Then

$$
\begin{aligned}
& \int_{M} \phi^{(k)} \cdot \psi^{(k)} \circ(I-T)^{m} \circ T^{n} d \mu=\sum_{j=0}^{m} \frac{m !}{j !(m-j) !}(-1)^{j} \int_{M} \phi^{(k)} \cdot \psi^{(k)} \circ T^{n+j} d \mu \\
& =\sum_{N_{1}, N_{2} \in \mathbb{Z}^{d}} \frac{1}{(2 \pi)^{d}} \int_{[-\pi, \pi]^{d}} e^{-i t \cdot\left(N_{2}-N_{1}\right)} \sum_{j=0}^{m} \frac{m !}{j !(m-j) !}(-1)^{j} \mathbb{E}_{\mu_{\Delta}}\left[P_{t}^{k}\left(\tilde{g}_{N_{2}}^{(k)} P_{t}^{n+j-2 k} P_{t}^{k} P^{k} \tilde{h}_{N_{1}}^{(k)}\right)\right] \\
& =\sum_{N_{1}, N_{2} \in \mathbb{Z}^{d}} \frac{1}{(2 \pi)^{d}} \int_{[-\pi, \pi]^{d}} e^{-i t \cdot\left(N_{2}-N_{1}\right)} \mathbb{E}_{\mu_{\Delta}}\left[P_{t}^{k}\left(\tilde{g}_{N_{2}}^{(k)}\left(I-P_{t}\right)^{m} P_{t}^{n-2 k} P_{t}^{k} P^{k} \tilde{h}_{N_{1}}^{(k)}\right)\right]
\end{aligned}
$$

Now, as seen in the proof of the first item of Proposition 7.4 combined with (136)

$$
\begin{aligned}
& \int_{M} \phi^{(k)} \cdot \psi^{(k)} \circ(I-T)^{m} \circ T^{n} d \mu=\frac{1}{(2 \pi)^{d} a_{n}^{d}} \sum_{N_{1}, N_{2} \in \mathbb{Z}^{d}} \int_{\left[-\beta a_{n}, \beta a_{n}\right]^{d}} e^{-i u \cdot\left(N_{2}-N_{1}\right) / a_{n}} \lambda_{u / a_{n}}^{n-2 k}\left(1-\lambda_{u / a_{n}}\right)^{m} \\
& \quad \times \mathbb{E}_{\mu_{\Delta}}\left[P_{u / a_{n}}^{k}\left(\tilde{g}_{N_{2}}^{(k)} \Pi_{u / a_{n}} P_{u / a_{n}}^{k} P_{u / a_{n}} \tilde{h}_{N_{1}}^{(k)}\right)\right] d u+O\left(\theta^{n}\left\|g_{N_{2}}\right\|_{\infty}\left\|h_{N_{1}}\right\|_{\infty}\right) \\
& =-\frac{(\log n+\log \log n)^{m}}{2^{m} a_{n}^{d+2 m}}\left(O\left((\log n)^{m-1}\right)+\sum_{N_{1}, N_{2} \in \mathbb{Z}^{d}} J_{0, m, n, 0,0} \mathbb{E}_{\mu_{\Delta}}\left[P_{u / a_{n}}^{k} \tilde{g}_{N_{2}}^{(k)}\right] \mathbb{E}_{\mu_{\Delta}}\left[P_{u / a_{n}}^{k} P_{u / a_{n}} \tilde{h}_{N_{1}}^{(k)}\right]\right),
\end{aligned}
$$

where we used (86), (95), (134), (87) and $\log \left(\left|a_{n} / u\right|\right)=\log a_{n}+O(\log |u|)$ exactly as we obtained (104) in the proof of Proposition 7.4. Moreover

$$
\begin{aligned}
\mathbb{E}_{\mu_{\Delta}}\left[P_{u / a_{n}}^{k} P_{u / a_{n}} \tilde{h}_{N_{1}}^{(k)}\right] & =\mathbb{E}_{\mu_{\Delta}}\left[\tilde{h}_{N_{1}}^{(k)}\right]+O\left(\frac{k}{a_{n}}\|\kappa\|_{L^{1}(\bar{\mu})}\left\|h_{N_{1}}\right\|_{\infty}\right) \\
& =\mathbb{E}_{\bar{\mu}}\left[h_{N_{1}}\right]+O\left(\frac{k}{a_{n}}\|\kappa\|_{L^{1}(\bar{\mu})}\left\|h_{N_{1}}\right\|_{(\eta)}\right), \\
& \text { and } \mathbb{E}_{\mu_{\Delta}}\left[P_{u / a_{n}}^{k} \tilde{g}_{N_{2}}^{(k)}\right]=\mathbb{E}_{\bar{\mu}}\left[g_{N_{2}}\right]+O\left(\frac{k}{a_{n}}\|\kappa\|_{L^{1}(\bar{\mu})}\left\|g_{N_{2}}\right\|_{(\eta)}\right),
\end{aligned}
$$

Combining this with (135), (138), with our summability assumptions and with (134), up to an error in $O\left(a_{n}^{-d-2 m}(\log n)^{m-1}\right)$, we obtain a dominating term in

$$
-\frac{(\log (n \log n))^{m}}{2^{m}(n \log n)^{\frac{d}{2}+m}}\left(I_{0, m, 0}(0)+I_{0, m+1,0}(0) \frac{\log \log n}{2 \log n}\right) \int_{M} \phi d \mu \int_{M} \psi d \mu .
$$

But, due to (101), $I_{0, k, 0}=\frac{\Delta^{k} \Phi(0)}{\sqrt{\operatorname{det} \Sigma^{2}}}$ and so

$$
\begin{aligned}
I_{0, k, 0} & =\sum_{i_{1}, \ldots, i_{k}=1}^{d} \frac{\partial^{2 k}}{\partial^{2} x_{i_{1}} \cdots \partial^{2} x_{i_{k}}} \frac{\Phi}{\sqrt{\operatorname{det} \Sigma^{2}}}(0)=\sum_{k_{1}+\cdots+k_{d}=k}^{d} \frac{k !}{k_{1} ! \ldots k_{d} !} \frac{\partial^{2 k}}{\partial^{2 k_{1}} x_{1} \cdots \partial^{2 k_{d} x_{d}}} \frac{\Phi}{\sqrt{\operatorname{det} \Sigma^{2}}}(0) \\
& =\sum_{k_{1}+\cdots+k_{d}=k}^{d} \frac{k ! \Phi(0)}{k_{1} ! \ldots k_{d} ! \sqrt{\operatorname{det} \Sigma^{2}}} \prod_{j=1}^{d}(-1)^{k_{j}} \mathbb{E}\left[Z_{j}^{2 k_{j}}\right]=\frac{(-1)^{k} \Phi(0) \mathbb{E}\left[\left(Z_{1}^{2}+\cdots+Z_{d}^{2}\right)^{k}\right]}{\sqrt{\operatorname{det} \Sigma^{2}}} \\
& =(-1)^{k} \Phi_{\Sigma^{2}}(0) d(d+2) \ldots(d+2 k-2),
\end{aligned}
$$

where $Z_{j}$ are independent standard gaussian random variables since $\frac{\partial^{2 k}}{\partial^{2 k} x_{j}} \Phi(x)=$ $P_{2 k}\left(x_{j}\right) \Phi(x)$, with $P_{2 k}$ is a polynomial such that $P_{2 k}(0)=\frac{\partial^{2 k}}{\partial^{2 k} x_{j}} \Phi(0)=\frac{(-1)^{k}(2 k) !}{2^{k} k !}=$ $(-1)^{k} \mathbb{E}\left[Z_{j}^{2 k}\right]$ (we use also the well-known moments of the chi-squared distribution of $Z_{1}^{2}+\cdots+Z_{d}^{2}$ ). So $I_{0, m, 0}(0)+I_{0, m+1,0}(0) \frac{\log \log n}{2 \log n}=I_{0, m, 0}(0)\left(1-\frac{(d+2 m) \log \log n}{2 \log n}\right)$ and we conclude by the comments after the statement of Theorem 2.5. 
- Let us prove item (b):

$$
\begin{aligned}
& \int_{M} \phi \cdot \psi \circ T^{n} d \mu=\int_{M} \phi^{(k)} \cdot \psi^{(k)} \circ T^{n} d \mu+O\left(\eta^{k}\right) \\
& =\sum_{N_{2} \in \mathbb{Z}^{d}} \mathbb{E}_{\mu_{\Delta}}\left[\tilde{h}_{0}^{k}\left(1_{\left\{\hat{\kappa}_{n} \circ f^{k}=N_{2}-N\right\}}+1_{\left\{\hat{\kappa}_{n} \circ f^{k}=N_{2}+N\right\}}-2 \times 1_{\left\{\hat{\kappa}_{n} \circ f^{k}=N\right\}}\right) \tilde{g}_{N_{2}}^{(k)} \circ f^{n}\right]+O\left(\eta^{k}\right) \\
& =\sum_{N_{2} \in \mathbb{Z}^{d}} \frac{1}{(2 \pi)^{d}} \int_{[-\pi, \pi]^{d}} e^{i t \cdot N_{2}}\left(e^{-i t \cdot N}+e^{i t \cdot N}-2\right) \mathbb{E}_{\mu_{\Delta}}\left[P_{t}^{k}\left(\tilde{g}_{N_{2}}^{(k)} P_{t}^{n-2 k} P_{t}^{k} P^{k} \tilde{h}_{0}^{(k)}\right)\right] d t, \\
& \quad \int_{M} \phi \cdot \psi \circ T^{n} d \mu=O\left(\theta^{n} \sum_{N_{2} \in \mathbb{Z}^{d}}\left\|g_{N_{2}}\right\|_{L^{q}\left(\mu_{\Delta}\right)}\left\|h_{0}\right\|_{\infty}\right) \\
& \quad+\sum_{N_{2} \in \mathbb{Z}^{d}} \frac{1}{(2 \pi)^{d}} \int_{[-\beta, \beta]^{d}} e^{i t \cdot N_{2}}\left(e^{-i t \cdot N}+e^{i t \cdot N}-2\right) \lambda_{t}^{n-2 k} \mathbb{E}_{\mu_{\Delta}}\left[P_{t}^{k}\left(\tilde{g}_{N_{2}}^{(k)} \Pi_{t} P_{t}^{k} P^{k} \tilde{h}_{0}^{(k)}\right)\right] d t .
\end{aligned}
$$

Now using the change of variable $t=u / a_{n}$, the expansion of the exponential and expansion (87) of $\Pi$ with $\gamma=1$, we obtain that $\int_{M} \phi . \psi \circ T^{n} d \mu$ is equal to

$$
\begin{aligned}
& \sum_{N_{2} \in \mathbb{Z}^{d}} \frac{1}{(2 \pi)^{d} a_{n}^{d+2}} \int_{\left[-\beta a_{n}, \beta a_{n}\right]^{d}} e^{i u \cdot N_{2} / a_{n}}(i u \cdot N)^{2} \lambda_{u / a_{n}}^{n-2 k} \mathbb{E}_{\mu_{\Delta}}\left[P_{u / a_{n}}^{k} \tilde{g}_{N_{2}}^{(k)}\right] \mathbb{E}_{\mu_{\Delta}}\left[P_{u / a_{n}}^{k} P^{k} \tilde{h}_{0}^{(k)}\right] d u \\
& +O\left(a_{n}^{-d-3} \sum_{N_{2} \in \mathbb{Z}^{d}}\left\|g_{N_{2}}\right\|_{L^{q_{0}}\left(\mu_{\Delta}\right)}\left\|h_{0}\right\|_{\infty}\right), \\
& \int_{M} \phi \cdot \psi \circ T^{n} d \mu=O\left((\log n) a_{n}^{-d-3} \sum_{N_{2} \in \mathbb{Z}^{d}}\left\|g_{N_{2}}\right\|_{L^{q}\left(\mu_{\Delta}\right)}\left\|h_{0}\right\|_{\infty}\right) \\
& +\sum_{N_{2} \in \mathbb{Z}^{d}} \frac{1}{(2 \pi)^{d} a_{n}^{d+2}} \int_{\left[-\beta a_{n}, \beta a_{n}\right]^{d}} e^{i u \cdot N_{2} / a_{n}}(i u \cdot N)^{2} \lambda_{u / a_{n}}^{n-2 k} \mathbb{E}_{\mu_{\Delta}}\left[\tilde{g}_{N_{2}}^{(k)}\right] \mathbb{E}_{\mu_{\Delta}}\left[\tilde{h}_{0}^{(k)}\right] d u,
\end{aligned}
$$

due to (139) and (140). Thus

$$
\begin{aligned}
& \int_{M} \phi . \psi \circ T^{n} d \mu=-\frac{1}{a_{n}^{d+2}} \sum_{N_{2} \in \mathbb{Z}^{d}} \mathbb{E}_{\mu_{\Delta}}\left[\tilde{g}_{N_{2}}^{(k)}\right] \mathbb{E}_{\mu_{\Delta}}\left[\tilde{h}_{0}^{(k)}\right] J_{2,0, n, N_{2}, N}+O\left((\log n) a_{n}^{-d-3}\right) \\
& =-\frac{1}{a_{n}^{d+2}} J_{2,0, n, 0, N} \sum_{N_{2} \in \mathbb{Z}^{d}} \mathbb{E}_{\mu_{\Delta}}\left[\tilde{g}_{N_{2}}^{(k)}\right] \mathbb{E}_{\mu_{\Delta}}\left[\tilde{h}_{0}^{(k)}\right]+O\left((\log n) a_{n}^{-d-3}+a_{n}^{-d-2-\delta}\right), \\
& \int_{M} \phi \cdot \psi \circ T^{n} d \mu=-\frac{1}{a_{n}^{d+2}} J_{2,0, n, 0, N} \sum_{N_{2} \in \mathbb{Z}^{d}} \mathbb{E}_{\bar{\mu}}\left[g_{N_{2}}\right] \mathbb{E}_{\bar{\mu}}\left[h_{0}\right]+O\left((\log n) a_{n}^{-d-3}+a_{n}^{-d-2-\delta}\right) \\
& =-\frac{1}{a_{n}^{d+2}} J_{2,0, n, 0, N} \int_{M} \psi d \mu \mathbb{E}_{\bar{\mu}}\left[h_{0}\right]+O\left((\log n) a_{n}^{-d-3}+a_{n}^{-d-2-\delta}\right),
\end{aligned}
$$

using (134) and then our definition of $k(n)$. We conclude by (134) and (105).

Acknowledgements. The research of FP was partially supported by the IUF, Institut Universitaire de France. The research of DT was partially supported by EPSRC grant EP/S019286/1. We wish also to thank the mathematical departments of the Universities of Brest and Exeter for their hospitality.

We thank the referees for their careful reading of the manuscript which helped us to substantially improve the presentation. 
Open Access This article is licensed under a Creative Commons Attribution 4.0 International License, which permits use, sharing, adaptation, distribution and reproduction in any medium or format, as long as you give appropriate credit to the original author(s) and the source, provide a link to the Creative Commons licence, and indicate if changes were made. The images or other third party material in this article are included in the article's Creative Commons licence, unless indicated otherwise in a credit line to the material. If material is not included in the article's Creative Commons licence and your intended use is not permitted by statutory regulation or exceeds the permitted use, you will need to obtain permission directly from the copyright holder. To view a copy of this licence, visit http://creativecommons.org/licenses/by/4.0/.

Publisher's Note Springer Nature remains neutral with regard to jurisdictional claims in published maps and institutional affiliations.

\section{Appendix A. Tail Probability of the Return Time to the Initial Cell}

This appendix is devoted to the proof of Theorem 2.1 (without assuming (12)). Since $\tau_{0}$ is constant along stable curves, there exists $\hat{\tau}_{0}: \Delta \rightarrow \mathbb{N}$ such that $\tau_{0} \circ \mathfrak{p}_{1}=\hat{\tau}_{0} \circ \mathfrak{p}_{2}$. We use the classical Dvoretzky and Erdös argument [14] combined with the estimates provided by our Proposition 3.1 and (3) (we do not need Proposition 3.3 here). Considering the last visit time $n$ to the 0 -cell before time $N$, we observe that

$$
1=\sum_{n=0}^{N} \bar{\mu}\left(\kappa_{n}=0, \tau_{0} \circ \bar{T}^{n}>N-n\right)=\sum_{n=0}^{N} \mathbb{E}_{\mu_{\Delta}}\left[\mathbf{1}_{\left\{\hat{\tau}_{0}>N-n\right\}} P^{n}\left(\mathbf{1}_{\left\{\hat{\kappa}_{n}=0\right\}}\right)\right] .
$$

Moreover, it follows from (15), (16), (17) and Proposition 3.1 that

$$
\begin{aligned}
& P^{n}\left(\mathbf{1}_{\left\{\hat{\kappa}_{n}=0\right\}}\right)-\bar{\mu}\left(\kappa_{n}=0\right)=\frac{1}{(2 \pi)^{d}} \int_{[-\pi, \pi]^{d}}\left(I d-\Pi_{0}\right) P_{t}^{n}(\mathbf{1}) d t \\
& \quad=\frac{1}{(2 \pi)^{d}} \int_{[-\beta, \beta]^{d}} \lambda_{t}^{n}\left(I d-\Pi_{0}\right) \Pi_{t}(\mathbf{1}) d t+O\left(\theta^{n}\right) \\
& \quad=O\left(\theta^{n}+\int_{[-\beta, \beta]^{d}} e^{-n a|t|^{2} \log \left(|t|^{-1}\right)}|t|^{\gamma} d t\right)=O\left(\theta^{n}+(n \log n)^{-\frac{d+\gamma}{2}}\right)
\end{aligned}
$$

in $L^{p^{\prime}}\left(\mu_{\bar{\Delta}}\right)$ with $p^{\prime}>1$ and $\gamma>1$ as in Proposition 3.1 , using $\Pi_{0}\left(\Pi_{0}^{\prime}(\mathbf{1})\right)=0$. Therefore

$$
1=\sum_{n=0}^{N}\left[\bar{\mu}\left(\tau_{0}>N-n\right) \bar{\mu}\left(\kappa_{n}=0\right)+\varepsilon_{n, N}\right]
$$

with $\left|\varepsilon_{n, N}\right|=O\left(\bar{\mu}\left(\tau_{0}>N-n\right)^{\frac{1}{q^{\prime}}}\left(\theta^{n}+(n \log n)^{-\frac{d+\gamma}{2}}\right)\right)$ uniformly in $(n, N)$ (with $\left.\frac{1}{q^{\prime}}+\frac{1}{p^{\prime}}=1\right)$. Since $(M, \mu, T)$ is recurrent, $\lim _{N \rightarrow+\infty} \bar{\mu}\left(\tau_{0}>N\right)=0$, so $\lim _{N \rightarrow+\infty} \sum_{n=0}^{N} \varepsilon_{n, N}=0$ and

$$
1=\lim _{N \rightarrow+\infty} \sum_{n=0}^{N} \bar{\mu}\left(\tau_{0}>N-n\right) \bar{\mu}\left(\kappa_{n}=0\right) .
$$


In particular $1 \geq \lim \sup _{N \rightarrow+\infty} \bar{\mu}\left(\tau_{0}>N\right) \sum_{n=0}^{N} \bar{\mu}\left(\kappa_{n}=0\right)$. It follows from $\bar{\mu}\left(\kappa_{n}=\right.$ $0) \sim \frac{\Phi_{\Sigma^{2}}(0)}{(n \log n)^{\frac{d}{2}}}([30])$ that $\sum_{n=0}^{N} \bar{\mu}\left(\hat{\kappa}_{n}=0\right) \sim \Phi_{\Sigma^{2}}(0) \mathfrak{e}_{N}$ with $\mathfrak{e}_{N}=\log \log N$ if $d=2$ and with $\mathfrak{e}_{N}=\sqrt{\frac{N}{\log N}}$ if $d=1$. Therefore we obtain the following upper bound:

$$
\limsup _{N \rightarrow+\infty} \Phi_{\Sigma^{2}}(0) \mathfrak{e}_{N} \bar{\mu}\left(\tau_{0}>N\right) \leq 1
$$

- Assumed $=2$. It remains to prove that the lower bound coincide with the above upperbound. To this end, starting from (141) observe that, for $0 \leq m_{N} \leq N$,

$$
\begin{aligned}
1 & \leq \liminf _{N \rightarrow+\infty}\left(\sum_{n=0}^{m_{N}-1} \bar{\mu}\left(\tau_{0}>N-n\right) \bar{\mu}\left(\kappa_{n}=0\right)+\sum_{n=m_{N}}^{N} \bar{\mu}\left(\kappa_{n}=0\right)\right) \\
& \leq \liminf _{N \rightarrow+\infty}\left(\bar{\mu}\left(\tau_{0}>N-m_{N}\right) \sum_{n=0}^{m_{N}-1} \bar{\mu}\left(\kappa_{n}=0\right)+\sum_{n=m_{N}}^{N} \bar{\mu}\left(\kappa_{n}=0\right)\right) .
\end{aligned}
$$

applying this with $N=N^{\prime}\left\lfloor\log N^{\prime}\right\rfloor$ and $m_{N}=N^{\prime}\left(\left\lfloor\log N^{\prime}\right\rfloor-1\right)$, we obtain

$$
1 \leq \liminf _{N \rightarrow+\infty} \bar{\mu}\left(\tau_{0}>N^{\prime}\right) \Phi_{\Sigma^{2}}(0) \log \log \left(N^{\prime}\right)+O\left(\left(\log N^{\prime}\right)^{-2}\right),
$$

since $\sum_{n=N^{\prime}\left(\left\lfloor\log N^{\prime}\right\rfloor-1\right)}^{N^{\prime}\left\lfloor\log N^{\prime}\right\rfloor} \bar{\mu}\left(\kappa_{n}=0\right)=O\left(\log \frac{\log \left(N^{\prime}\left\lfloor\log N^{\prime}\right\rfloor\right)+\log \left(1-\frac{1}{\left\lfloor\log N^{\prime}\right\rfloor}\right)}{\log \left(N^{\prime}\left\lfloor\log N^{\prime}\right\rfloor\right)}\right)=$ $O\left(\left(\log N^{\prime}\right)^{-2}\right)$ and $\log \log m_{N} \sim \log \log N^{\prime}$. Combining this with (142), we finally obtain (10).

- Assumed $=1$. Upper bound (142) ensures that the sequence of non increasing functions $\left(\left(\mathfrak{e}_{N} \bar{\mu}\left(\tau_{0}>x N\right)\right)_{x \in(0, \infty)}\right)_{N \geq 1}$ admits limit points for the pointwise convergence except at discontinuity points of the limit. Consider a subsequence indexed by $\left(N_{k}\right)_{k}$ converging to a function $\psi$. It follows from (141) that, for all $y \in(0, \infty)$,

$$
\lim _{N \rightarrow+\infty} \int_{-1}^{\lfloor N y\rfloor} \bar{\mu}\left(\tau_{0}>\lfloor N y\rfloor-x\right) \bar{\mu}\left(\kappa_{\lceil x\rceil}=0\right) d x=1 .
$$

Note that $\int_{[-1, \varepsilon N] \cup[\lfloor N y\rfloor-N \varepsilon,\lfloor N y\rfloor]} \bar{\mu}\left(\tau_{0}>\lfloor N y\rfloor-x\right) \bar{\mu}\left(\kappa_{\lceil x\rceil}=0\right) d x$ is less than

$$
\sqrt{\frac{\log (N / 2)}{N / 2}} c^{\prime} \mathfrak{e}_{\varepsilon N}+\sum_{k=1}^{N \varepsilon} \sqrt{\frac{\log (k)}{k}} \frac{c^{\prime}}{\sqrt{(N / 2) \log (N / 2)}} \leq c^{\prime \prime} \sqrt{\frac{\varepsilon N \log (N)}{N \log (\varepsilon N)}}
$$

which, combined with (143) leads to

$$
\limsup _{N \rightarrow+\infty}\left|\int_{\varepsilon N}^{\lfloor N y\rfloor-N \varepsilon} \bar{\mu}\left(\tau_{0}>\lfloor N y\rfloor-x\right) \bar{\mu}\left(\kappa_{\lceil x\rceil}=0\right) d x-1\right|=O(\sqrt{\varepsilon}) .
$$

But, for every $\varepsilon>0, \int_{\varepsilon N}^{\lfloor N y\rfloor-N \varepsilon} \bar{\mu}\left(\tau_{0}>\lfloor N y\rfloor-x\right) \bar{\mu}\left(\kappa_{\lceil x\rceil}=0\right) d x$ is equal to

$$
\int_{\varepsilon}^{(\lfloor N y\rfloor-N \varepsilon) / N} \mathfrak{e}_{N} \bar{\mu}\left(\tau_{0}>\lfloor N y\rfloor-N u\right)\left(\sqrt{N \log N} \bar{\mu}\left(\kappa_{\lceil N u\rceil}=0\right)\right) d u
$$




$$
\rightarrow \int_{\varepsilon}^{y-\varepsilon} \psi(y-u) \frac{\Phi_{\Sigma^{2}}(0)}{\sqrt{u}} d u, \quad \text { as } N=N_{k} \rightarrow+\infty,
$$

due to the dominated convergence theorem since (142) and (3) ensure that

$$
N \bar{\mu}\left(\tau_{0}>\lfloor N y\rfloor-N u\right) \bar{\mu}\left(\kappa_{\lceil N u\rceil}=0\right) \leq c N \frac{1}{\mathfrak{e}_{N_{\varepsilon}} \sqrt{N \varepsilon \log (N \varepsilon)}}=O\left(\varepsilon^{-1}\right) .
$$

From which we conclude that $\int_{0}^{y} \psi(y-u) \frac{\Phi_{\Sigma^{2}}(0)}{\sqrt{u}} d u=1$ for any $y \in(0, \infty)$. Observe that $\psi_{0}(z):=\frac{1}{a \sqrt{z}}$ with $a=\Phi_{\Sigma^{2}}(0) \int_{0}^{1} \frac{d t}{\sqrt{t(1-t)}}$ is a solution of $\int_{0}^{y} \psi(y-$ u) $\frac{\Phi_{\Sigma^{2}}(0)}{\sqrt{u}} d u=1$. Recall that $\int_{0}^{1} \frac{d t}{\sqrt{t(1-t)}}=\pi$ (using for example the Euler Beta function), so $a=\pi \Phi_{\Sigma^{2}}(0)=\sqrt{\frac{\pi}{2 \Sigma^{2}}}$. Thus, for all $y \in(0, \infty), \int_{0}^{y}\left(\psi-\psi_{0}\right)(y-$ u) $\frac{\Phi_{\Sigma^{2}}(0)}{\sqrt{u}} d u=0$. Moreover, due to (142), $\psi(x) \leq C / \sqrt{x}$ and thus $\psi-\psi_{0}$ is integrable. We conclude by the Titchmarsh's Convolution Theorem (see e.g. [13]) that $\psi-\psi_{0} \equiv 0$. Thus $\psi_{0}$ is the unique limit point and so we have proved (11).

\section{Appendix B. Justifying Equation (40)}

Let $v: \Delta \rightarrow \mathbb{R}$ satisfying the assumptions of Lemma 5.8 and $w \in \mathcal{B}_{1}$. Using the pointwise formula $R\left(\hat{\kappa}_{\sigma} v w\right)(y)=\sum_{a \in \mathcal{A}} e^{\chi\left(y_{a}\right)} w\left(y_{a}\right)\left(v \hat{\kappa}_{\sigma}\right)\left(y_{a}\right)$ (and in particular (35)), the arguments used in the proof of [20, Proposition 12.1] show that the $\|\cdot\|_{\mathcal{B}_{0}}$ norm of $R\left(v \hat{\kappa}_{\sigma} \cdot\right)$ is bounded by $\left\|v \hat{\kappa}_{\sigma}\right\|_{L^{1}\left(\mu_{Y}\right)}$. We recall the main inequalities, which in turn will help us justify equations (40). An important assumption used throughout [20, Proof of Proposition 12.1] is [20, Assumption (A1)]. In our case, $v \hat{\kappa}_{\sigma}$ is constant on the partition elements $a \in \mathcal{Y}$ and thus, it automatically satisfies [20, Assumption (A1)]. In our case, [20, Assumption (A1)] translates into $\left|\sup _{a}\left(v \hat{\kappa}_{\sigma}\right)-\inf _{a}\left(v \hat{\kappa}_{\sigma}\right)\right| \leq C \inf _{a}(v \hat{\kappa})_{\sigma}$, for all $a \in \mathcal{Y}$ and some $C>0$. This allows for a direct application of the arguments in [20, Proof of Proposition 12.1] . By the argument [20, Proof of Proposition 12.1 a)] with $\varphi \in\left\{v \hat{\kappa}_{\sigma}, v 1_{Y}\right\}$, we have that for some $C>0$,

$$
\|R(\varphi w)\|_{\infty} \leq C\|w\|_{\infty}\left\|v \hat{\kappa}_{\sigma}\right\|_{L^{1}\left(\mu_{Y}\right)} .
$$

since $\varphi$ is constant on the partition elements $a \in \mathcal{Y}$. Recall that $|\cdot|_{\mathcal{B}_{0}}$ is the seminorm used in defining the norm $\|\cdot\|_{\mathcal{B}_{0}}$. A simplified version of [20, Proof of Proposition 12.1 b)] (which can deal the $p$ - derivative, for $p \in(1,2)$, in $t$ of $R\left(e^{i t \hat{\kappa}_{\sigma}} v\right)$ ) with $\varphi \in$ $\left\{v 1_{Y}, v \hat{\kappa}_{\sigma}, 1_{\{\sigma=n\}} v \hat{\kappa}_{\sigma}, 1_{\{\sigma=n\}}\left(e^{i t \hat{\kappa}_{\sigma}}-1-i t \hat{\kappa}_{\sigma}\right) v\right\}$ ensures that

$$
\|R(\varphi w)\|_{\mathcal{B}_{0}} \leq C\|w\|_{\mathcal{B}_{0}}\|\varphi\|_{L^{1}\left(\mu_{Y}\right)},
$$

since the function $\varphi$ is constant on the partition elements $a \in \mathcal{A}$. In particular, $\left\|R\left(1_{\{\sigma=n\}} v\right) w\right\|_{\mathcal{B}_{0}} \leq C\|w\|_{\mathcal{B}_{0}}\left\|1_{\{\sigma=n\}} 1_{Y} v\right\|_{L^{1}\left(\mu_{Y}\right)}$.

\section{Appendix C. On the Constant $c_{0}(v, w)$}

We show that the sums in the expression of $c_{0}(v, w)$ in Lemma 5.12 are absolutely convergent. Since we do not know if $\hat{\kappa}$ is in $\mathcal{B}$, our strategy is to use $1_{(a, l)}$ and exploit that $\hat{\kappa}$ is constant on $(a, l)$. The argument below is delicate since the $\mathcal{B}$-norm of $1_{(a, l)}$ increases exponentially fast in $\sigma(a)$. 
Lemma C.1. $\sup _{a \in Y, l \in\{0, \ldots, \sigma(a)-1\}}\left\|P_{0}^{\sigma(a, l)}\left(\cdot 1_{(a, l)}\right)\right\|_{\mathcal{L}\left(\mathcal{B}_{0} \rightarrow \mathcal{B}\right)}<\infty$.

Proof. Let $y \in Y \times\left\{\ell_{0}\right\}$ with $\ell_{0} \neq 0$ and $w \in \mathcal{B}_{0}$. We have $P^{\sigma(a, l)}\left(w 1_{(a, l)}\right)(y)=$ 0 and so $P^{\sigma(a, l)}\left(w 1_{(a, l)}-\mu_{\Delta}\left(w 1_{(a, l)}\right)\right)(y)=-\mu_{\Delta}\left(w 1_{(a, l)}\right)$. Let $y \in Y \times\{0\}$, we have $P^{\sigma(a, l)}\left(w 1_{(a, l)}\right)(y)=\chi\left(y_{a}\right) w\left(f^{l} y_{a}\right)$, where $y_{a}$ is the unique element of $a \cap$ $f^{-\sigma(a)}(\{y\})=a \cap F^{-1}(\{y\})$. Thus,

$$
\begin{aligned}
& \sup _{x, y \in Y \times\{0\}} \frac{P^{\sigma(a, l)}\left(w 1_{(a, l)}\right)(x)-P^{\sigma(a, l)}\left(w 1_{(a, l)}\right)(y)}{\beta^{s_{0}(x, y)}} \\
& \leq \sup _{x, y \in Y} \frac{e^{-\alpha_{\sigma(a, l)}\left(f^{l}\left(x_{a}\right)\right)}-e^{-\alpha_{\sigma(a, l)}\left(f^{l}\left(y_{a}\right)\right)}}{\beta^{s_{0}(x, y)}}\left\|w 1_{(a, l)}\right\|_{\infty}+\sup _{x, y \in Y} \frac{w\left(f^{l}\left(x_{a}\right)\right)-w\left(f^{l}\left(y_{a}\right)\right)}{\beta^{s_{0}(x, y)}} \sup _{a} \chi \\
& \leq\|w\|_{\mathcal{B}_{0}} 2 \sup _{x \in Y} e^{-\alpha_{\sigma_{a}}\left(f^{l}\left(x_{a}\right)\right)}\left(1+\sum_{k=0}^{\sigma(a, l)-1} \frac{\left|\alpha\left(f^{l+k}\left(x_{a}\right)\right)-\alpha\left(f^{l+k}\left(y_{a}\right)\right)\right|}{\beta^{s_{0}(x, y)}}\right) \leq C\|w\|_{\mathcal{B}_{0}} \mu_{\Delta}(a),
\end{aligned}
$$

where we have used that $s_{0}(x, y)=s_{0}\left(x_{a}, y_{a}\right)-\sigma(a, l) \leq s_{0}\left(x_{a}, y_{a}\right)$, that $|\alpha(x)-\alpha(y)| \leq C_{1}^{\prime} \beta^{s_{0}(x, y)}$ as soon as $s_{0}(x, y) \geq 1$ and finally the distorsion bounds. Moreover, as required,

$$
\sup _{(x, y) \in Y \times\{0\}} P^{\sigma(a, l)}\left(w 1_{(a, l)}\right)(x) \leq\|w\|_{\infty} \sup _{(a, l)} e^{\alpha_{\sigma(a, l)}} \leq C\|w\|_{\infty} \mu_{\Delta}(a) .
$$

Lemma C.2. There exist $\theta_{0}, \theta_{2} \in(0,1)$ so that $\left|\int_{\Delta} \hat{\kappa} 1_{Y} \circ f^{j} d \mu_{\Delta}\right|=O\left(\theta_{0}^{j}\right)$ and so that $w \in \mathcal{B},\left|\int_{\Delta} \hat{\kappa} \circ f^{j} w d \mu_{\Delta}\right|=O\left(\theta_{2}^{j}\|w\|_{\mathcal{B}}\right)$.

Proof. Let $p_{1}<2$ and $\epsilon \in(0,1)$. Note that $\mu_{\Delta}(\sigma \geq m) \leq \sum_{k \geq m} k \mu_{Y}(\sigma \geq k) \ll$ $\theta_{1}^{m(1-\epsilon)}$. Using Hölder inequality and Lemma C.1, we compute that

$$
\begin{aligned}
& \int_{\Delta} \hat{\kappa} 1_{Y} \circ f^{j} d \mu_{\Delta}=\int_{\Delta}\left(\hat{\kappa} 1_{\{\sigma<j / 2\}}-\mu_{\Delta}\left(\hat{\kappa} 1_{\{\sigma<j / 2\}}\right) 1_{Y} \circ f^{j} d \mu_{\Delta}+2\|\hat{\kappa}\|_{L^{p_{1}}\left(\mu_{\Delta}\right)}\left(\mu_{\Delta}(\sigma \geq j / 2)\right)^{\frac{p_{1}-1}{p_{1}}}\right. \\
& =\sum_{m \in \mathbb{Z}^{d}} m \sum_{(a, l): \hat{\kappa}(a, l)=m, \sigma(a, l)<j / 2} \int_{\Delta} P^{j}\left(1_{(a, l)}-\mu_{\Delta}((a, l))\right) 1_{Y} d \mu_{\Delta}+O\left(\theta_{1}^{\frac{(1-\epsilon) j\left(p_{1}-1\right)}{2 p_{1}}}\right) .
\end{aligned}
$$

Thus $\int_{\Delta} \hat{\kappa} 1_{Y} \circ f^{j} d \mu_{\Delta}$ is dominated, up to a multiplicative constant, by

$$
\sum_{m \in \mathbb{Z}^{d}}|m| \sum_{(a, l): \hat{\kappa}(a, l)=m, \sigma(a, l)<j / 2} \theta^{j-\sigma(a, l)} \mu_{\Delta}((a, l))+O\left(\theta_{1}^{\frac{(1-\epsilon) j\left(p_{1}-1\right)}{2 p_{1}}}\right) \ll \theta^{j / 2}+\theta_{1}^{\frac{(1-\epsilon) j\left(p_{1}-1\right)}{2 p_{1}}} .
$$

If $w \in \mathcal{B}$ and $p>2$, (16) ensures that $\left|\int_{\Delta} \hat{\kappa} \circ f^{j} \cdot w d \mu_{\Delta}\right| \leq C\|\hat{\kappa}\|_{L^{\frac{p}{p-1}\left(\mu_{\Delta}\right)}} \theta^{j}\|w\|_{\mathcal{B}}$.

We end with a technical lemma (of flavour somewhat similar to that of Lemma C.2) used in the proof of in Lemma 5.12.

Lemma C.3. Let $\delta$ as in the proof of Lemma 5.12. There exists $C>0$ so that, for any $v \in L^{b}\left(\mu_{\Delta}\right)$ and $w \in \mathcal{B}_{0}$ satisfy the assumptions on $v, w$ in the statement of Proposition 5.3 and any $\xi \in \mathbb{C}$ so that $|\xi|<(1-\delta)^{-1}$,

$$
\sum_{(a, \ell)} \sum_{r \geq 0}|\xi|^{-r} \int_{\Delta}\left|P_{0}^{r}\left(v w 1_{(a, \ell)}-\int_{(a, l)} v w d \mu_{\Delta}\right)\left(\hat{\kappa} 1_{Y} \circ f^{j}\right)\right| d \mu_{\Delta} \leq C\|w\|_{\mathcal{B}_{0}}\|v\|_{L^{b}\left(\mu_{\Delta}\right)},
$$


Proof. Note that $\left\|P_{0}^{\sigma(a)-\ell}\left(w v 1_{(a, \ell)}-\mu_{\Delta}\left(w v 1_{(a, \ell)}\right)\right)\right\|_{\mathcal{B}} \ll\|w\|_{\mathcal{B}_{0}} \mu_{\Delta}\left(|v| 1_{(a, \ell)}\right)$ since $v$ is is constant on the $a \in \mathcal{Y}$ and due to Lemma C.1. Thus,

$$
\begin{aligned}
J_{1} & :=\sum_{(a, \ell)} \sum_{r \geq \sigma(a)-\ell}|\xi|^{-r} \int_{\Delta}\left|P_{0}^{r}\left(v w 1_{(a, \ell)}-\int_{(a, l)} v w d \mu_{\Delta}\right)\left(\hat{\kappa} 1_{Y} \circ f^{j}\right)\right| d \mu_{\Delta} \\
& \leq \sum_{(a, \ell)} \sum_{r \geq \sigma(a)-\ell}(1-\delta)^{-r} C_{0} \theta^{r-\sigma(a)+\ell}\|w\|_{\mathcal{B}_{0}} \mu_{\Delta}\left(|v| 1_{(a, \ell)}\right)\|\hat{\kappa}\|_{L^{p /(p-1)}\left(\mu_{\Delta}\right)} \\
J_{1} & \ll \sum_{(a, \ell)}(1-\delta)^{\ell-\sigma(a)}\|w\|_{\mathcal{B}_{0}}\left\|v 1_{(a, \ell)}\right\|_{L^{1}\left(\mu_{\Delta}\right)} \leq\left(\sum_{(a, \ell)}(1-\delta)^{\frac{p}{p-1}(\ell-\sigma(a))} \mu_{\Delta}(a, \ell)\right)^{\frac{p-1}{p}}\|w\|_{\mathcal{B}_{0}}\|v\|_{L^{p}\left(\mu_{\Delta}\right)} \\
& \ll\left(\sum_{a}(1-\delta)^{-\frac{p}{p-1} \sigma(a)} \mu_{Y}(a)\right)^{\frac{p-1}{p}}\|w\|_{\mathcal{B}_{0}}\|v\|_{L^{p}\left(\mu_{\Delta}\right)} \ll\left(\sum_{m \geq 1}(1-\delta)^{-\frac{p}{p-1} m} \theta_{1}^{m}\right)^{\frac{p-1}{p}}\|w\|_{\mathcal{B}_{0}}\|v\|_{L^{p}\left(\mu_{\Delta}\right)} .
\end{aligned}
$$

In the previous displayed chain of equations we have used that $\mu_{Y}(\sigma \geq m) \ll \theta_{1}^{m}$, $(1-\delta)^{-1} \theta<1$ and that $(1-\delta)^{-\frac{p}{p-1}} \theta_{1}<1$. This takes care of the tails of the above sums. Next, we deal with the partials sums (up to $\sigma$ ) in the chain of equations below (61) leading to $U_{1, N^{\prime}}(t)=0$.

$$
\begin{aligned}
J_{2} & :=\sum_{(a, \ell)} \sum_{r=0}^{\sigma(a)-\ell}|\xi|^{-r} \int_{\Delta}\left|P_{0}^{r}\left(v w 1_{(a, \ell)}-\mu_{\Delta}\left(v w 1_{(a, l)}\right)\right)\left(\hat{\kappa} 1_{Y} \circ f^{j}\right)\right| d \mu_{\Delta} \\
& \ll \sum_{(a, \ell)}(1-\delta)^{\ell-\sigma(a)}\left\|v w 1_{(a, \ell)}-\int_{(a, l)} v w d \mu_{\Delta}\right\|_{L^{p}\left(\mu_{\Delta}\right)}\|\hat{\kappa}\|_{L^{\frac{p}{p-1}}\left(\mu_{\Delta}\right)}, \\
J_{2} & \ll \sum_{(a, \ell)}(1-\delta)^{\ell-\sigma(a)}\|w\|_{\mathcal{B}_{0}}\left|C_{(a, \ell)}\right|\left(\mu_{\Delta}((a, \ell))\right)^{\frac{1}{b}+\left(\frac{1}{p}-\frac{1}{b}\right)} \\
& \ll\left(\sum_{(a, \ell)}(1-\delta)^{\frac{b}{b-1}(\ell-\sigma(a))}\left(\mu_{\Delta}((a, \ell))\right)^{\frac{b}{b-1}\left(\frac{1}{p}-\frac{1}{b}\right)}\right)^{\frac{b-1}{b}}\|w\|_{\mathcal{B}_{0}}\left(\sum_{(a, \ell)}\left|C_{(a, \ell)}\right|^{b}\left(\mu_{\Delta}((a, \ell))\right)\right)^{\frac{1}{b}} .
\end{aligned}
$$

It follows that $J_{2} \ll\left(\sum_{a}(1-\delta)^{-\frac{b}{b-1} \sigma(a)}\left(\mu_{Y}(a)\right)^{\frac{b}{b-1}\left(\frac{1}{p}-\frac{1}{b}\right)}\right)^{\frac{b-1}{b}}\|w\|_{\mathcal{B}_{0}}\|v\|_{L^{b}\left(\mu_{\Delta}\right)}$ and finally

$$
J_{2} \ll\left(\sum_{m \geq 1}(1-\delta)^{-\frac{b}{b-1} m} \theta_{1}^{m \frac{b}{b-1}\left(\frac{1}{p}-\frac{1}{b}\right)}\right)^{\frac{b-1}{b}}\|w\|_{\mathcal{B}_{0}}\|v\|_{L^{b}\left(\mu_{\Delta}\right)} .
$$

\section{References}

1. Aaronson, J., Denker, M.: A local limit theorem for stationary processes in the domain of attraction of a normal distribution. In: N. Balakrishnan, I.A. Ibragimov, V.B. Nevzorov, eds., Asymptotic methods in probability and statistics with applications. International conference, St. Petersburg, Russia, 1998, Basel: Birkhäuser, pp. 215-224 (2001)

2. Bálint, P., Gouëzel, S.: Limit theorems in the stadium billiard. Commun. Math. Phys. 263, 461-512 (2006)

3. Bleher, P.M.: Statistical properties of two-dimensional periodic Lorentz gas with infinite horizon. J. Stat. Phys. 66(1), 315-373 (1992) 
4. Bruin, H., Melbourne, I., Terhesiu, D.: Rates of mixing for nonMarkov infinite measure semiflows. Trans. Am. Math. Soc. 371, 7343-7386 (2019)

5. Bunimovich, L.A., Sinai, YaG: Statistical properties of Lorentz gas with periodic configuration of scatterers. Commun. Math. Phys. 78, 479-497 (1981)

6. Bunimovich, L.A., Sină̌, Ya.G., Chernov, N.I.: Statistical properties of two-dimensional hyperbolic billiards. (Russian) Uspekhi Mat. Nauk 46 (1991), no. 4(280), 43-92, 192; translation in Russian Math. Surveys 46 (1991), no. 4, 47-106

7. Chernov, N.: Decay of correlations and dispersing billiards. J. Statist. Phys. 94, 513-556 (1999)

8. Conze, J.-P.: Sur un critère de récurrence en dimension 2 pour les marches stationnaires, applications. Ergod. Theory Dyn. Syst. 19, 1233-1245 (1999)

9. Dolgopyat, D., Nándori, P.: On mixing and the local central limit theorem for hyperbolic flows. Erg. Theory Dyn. Syst. (to appear) arXiv:1710.08568

10. Dolgopyat, D., Nándori, P.: Infinite measure renewal theorem and related results. Bull. Lond. Math. Soc. (to appear) arXiv:1709.04074

11. Dolgopyat, D., Nándori, P., Pène, F.: Asymptotic expansion of correlation functions for $\mathbb{Z}^{d}$ covers of hyperbolic flows. arXiv: 1908.11504

12. Dolgopyat, D., Szász, D., Varjú, T.: Recurrence properties of planar Lorentz process. Duke Math. J. 142(2), 241-281 (2008)

13. Doss, R.: an elementary proof of Titchmarsh's convolution theorem. Proc. AMS 104(1), 181-184 (1988)

14. Dvoretzky, A., Erdös, P.: Some problems on random walk in space. In: Proceedings of 2nd Berkeley Symposium. Math. Statist. Probab., Berkeley, CA: University of California Press, pp. 353-367 (1951)

15. Gouëzel, S.: Correlation asymptotics from large deviations in dynamical systems with infinite measure. Colloq. Math. 125, 193-212 (2011)

16. Keller, G., Liverani, C.: Stability of the spectrum for transfer operators. Annali della Scuola Normale Superiore di Pisa, Classe di Scienze 19, 141-152 (1999)

17. Liverani, C., Saussol, B., Vaienti, S.: A probabilistic approach to intermittency. Ergod. Theory Dyn. Syst. 19, 671-683 (1999)

18. Lorentz, H.A.: The motion of electrons in metallic bodies, Koninklijke Nederlandse Akademie van Wetenschappen (KNAW). Proc. Sect. Sci. 7(2), 438-593 (1905)

19. Melbourne, I., Terhesiu, D.: Operator renewal theory and mixing rates for dynamical systems with infinite measure. Invent. Math. 1, 61-110 (2012)

20. Melbourne, I., Terhesiu, D.: Operator renewal theory for continuous time dynamical systems with finite and infinite measure. Monatsh. Math. 182, 377-431 (2017)

21. Pène, F.: Planar Lorentz process in a random scenery. Annales de l'Institut Henri Poincaré, Probabilités et Statistiques 45(3), 818-839 (2009)

22. Pène, F., Saussol, B.: Back to balls in billiards. Commun. Math. Phys. 293, 837-866 (2010)

23. Pène, F.: Mixing and decorrelation in infinite measure: the case of the periodic Sinai Billiard. Annales de l'Institut Henri Poincaré Probab. Stat. 55(1), 378-411 (2019)

24. Pène, F., Thomine, D.: Potential kernel, hitting probabilities and distributional asymptotics. Erg. Theory Dyn. Syst. (In Press). arXiv:1702.06625

25. Pène, F., Thomine, D.: Central limit theorems for the $\mathbb{Z}^{2}$ periodic Lorentz gas. Isr. J. Math. (to appear). arXiv: 1909.05514

26. Sarig, O.M.: Subexponential decay of correlations. Invent. Math. 150, 629-653 (2002)

27. Schmidt, K.: On joint recurrence. C. R. Acad. Sci. Paris Série I 327, 837-842 (1998)

28. Sinai, YaG: Dynamical systems with elastic reflections. Russ. Math. Surv. 25(1), 137-189 (1970)

29. Szász, D., Varjú, T.: Local limit theorem for the Lorentz process and its recurrence in the plane. Ergod. Theory Dyn. Syst. 24, 254-278 (2004)

30. Szász, D., Varjú, T.: Limit laws and recurrence for the planar Lorents process with infinite horizon. J. Stat. Phys. 129, 59-80 (2007)

31. Terhesiu, D.: Mixing rates for intermittent maps of high exponent. Probab. Theory Relat. Fields 166, 1025-1060 (2016)

32. Young, L.-S.: Statistical properties of dynamical systems with some hyperbolicity. Ann. Math. 147, 585650 (1998)

Communicated by C. Liverani 